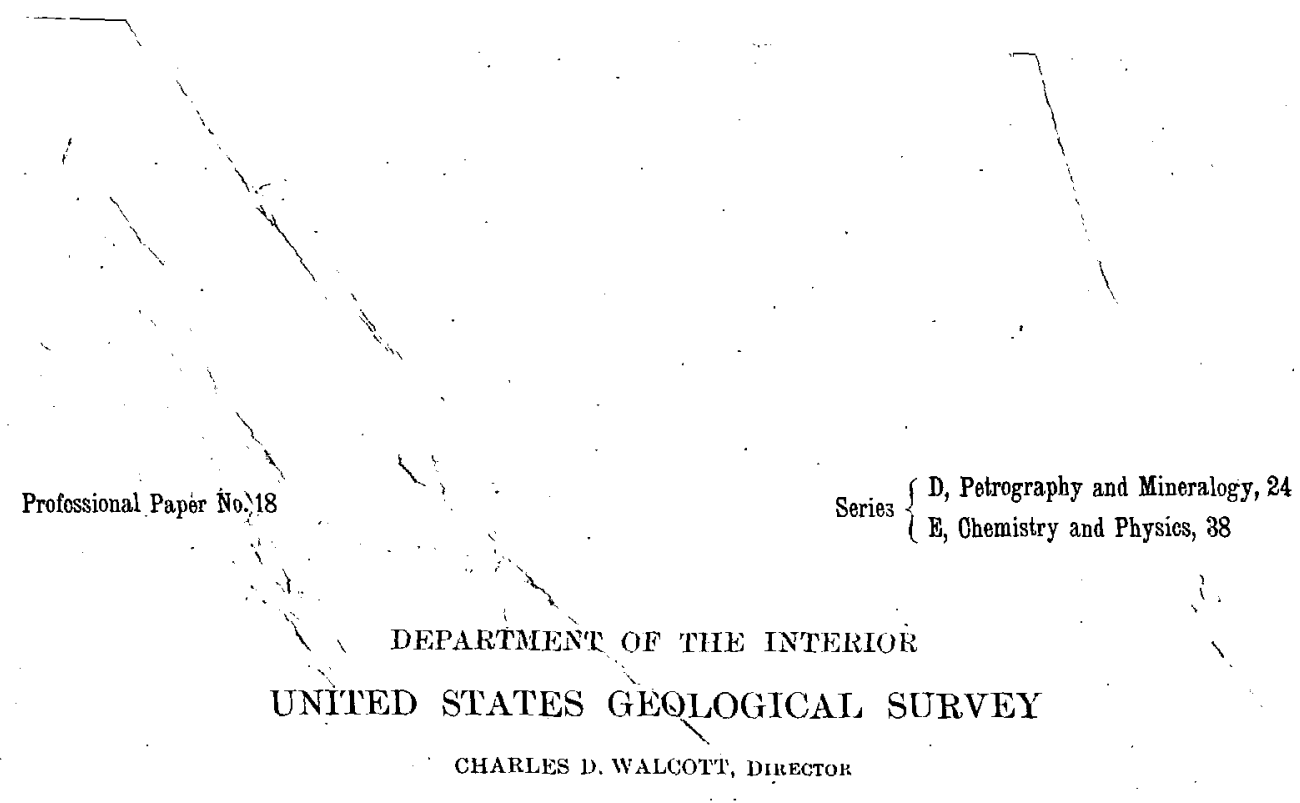

\title{
CHEMICAL COMPOSITION OF IGNEOUS ROCKS
}

EXPRESSED BY MEANS OF DIAGRAMS

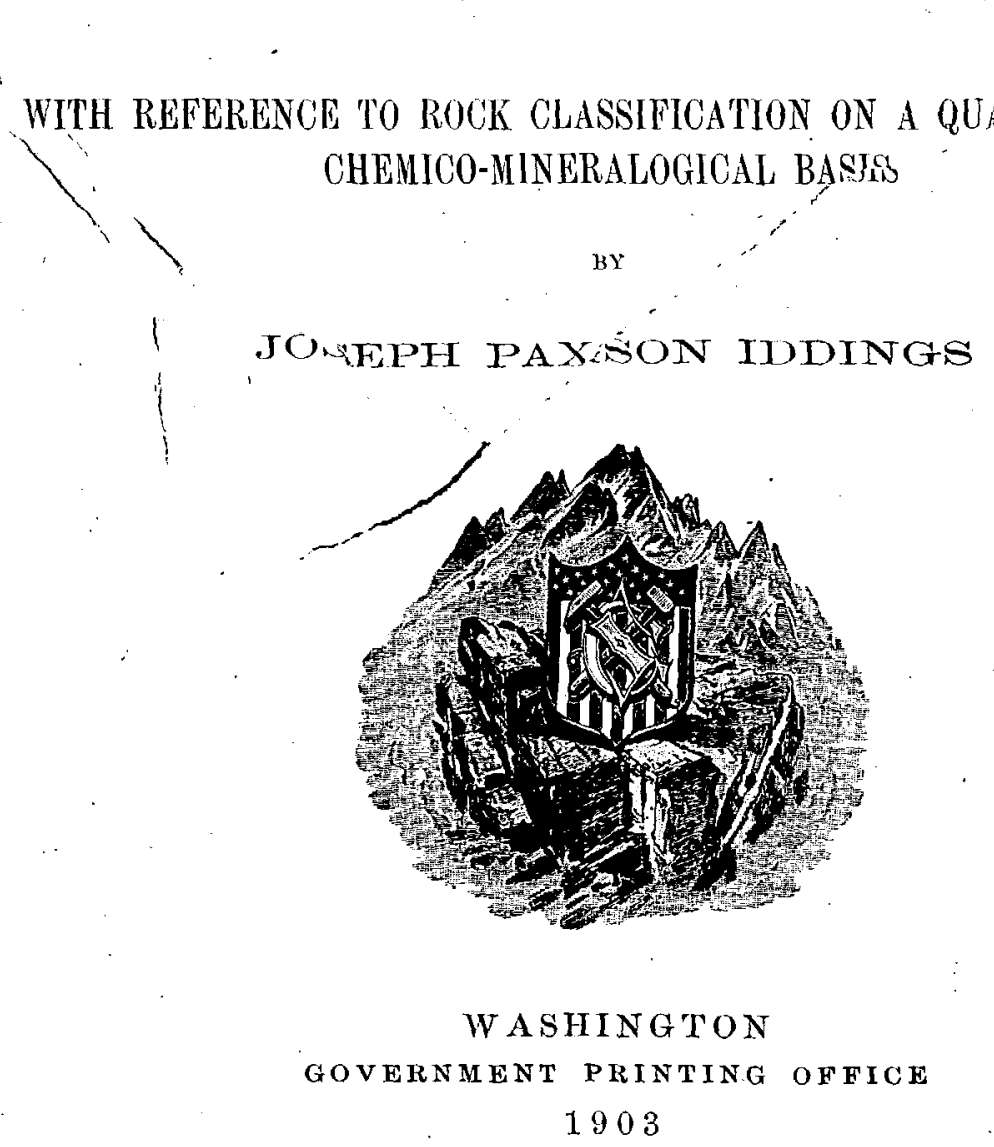





\section{ONTENTS.}

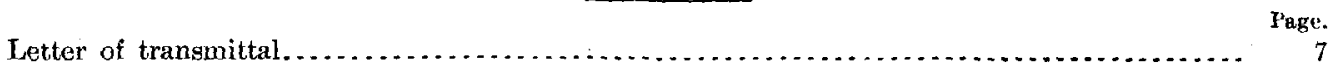

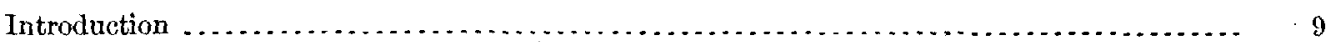

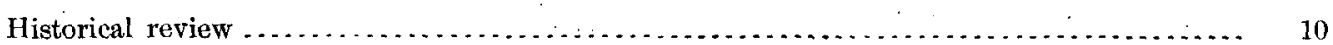

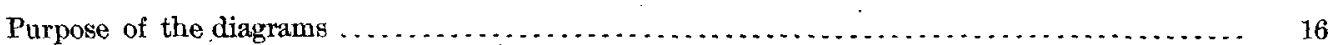

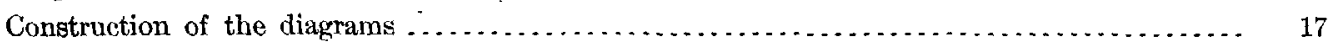

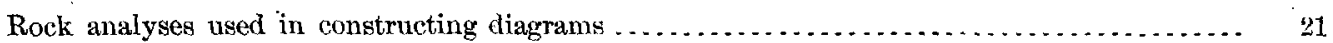

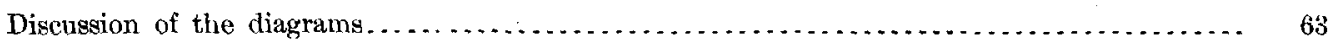

General diagrams. . . . . . . . . .

Variations in composition. . . . . .

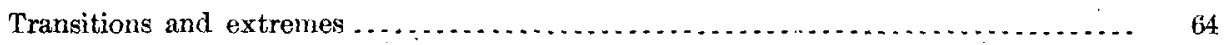

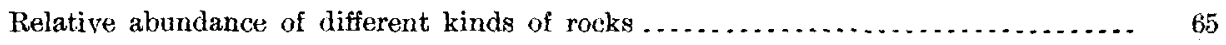

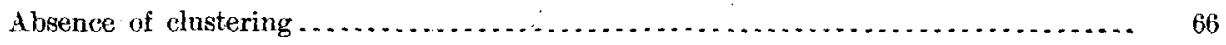

Similarity of rocks from different petrographical provinces $\ldots \ldots \ldots \ldots \ldots \ldots \ldots \ldots 6.66$

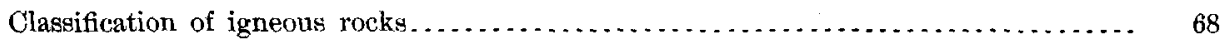

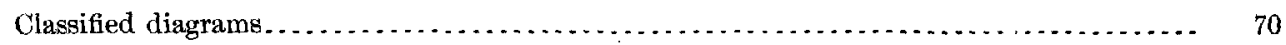

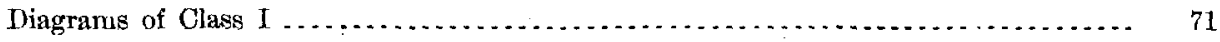

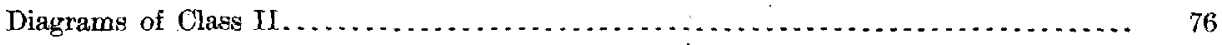

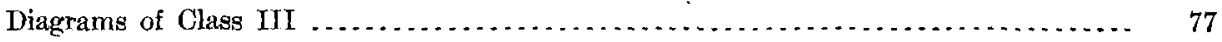

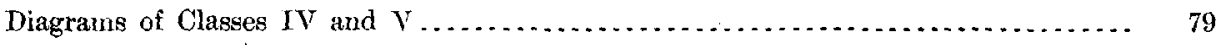

Correlation of igneous rocks classified by the quantitative and the qualitative systems..... 81 



\section{L L U S T R A T I O N S.}

Plate I. General diagram showing comparative values of chief chemical components of over 950 igneous rocks, and composite diagram showing distribution of Classes I, II, III, and IV-V.

Diagram showing limits of orders and rangs for theoretically pure soda-salic rocks

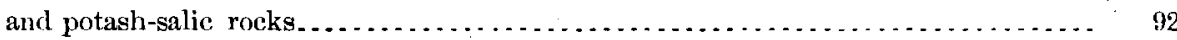

III. Diagrams showing chemical variations for rocks of Class $1 \ldots \ldots \ldots \ldots \ldots \ldots \ldots \ldots \ldots$

IV. Diagrams showing chemical variations for rocks of Class II $\ldots \ldots \ldots \ldots \ldots \ldots \ldots \ldots, \quad 92$

V. Diagrams showing distribution of analyses with respect to silici and alkali-silica

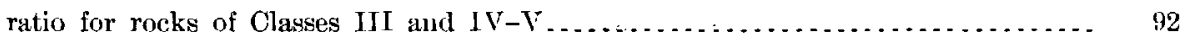

VI. Diagrams showing chemical variation for rocks of Class III . . . . . . . . . . . . 92

VII. Diagrams showing chemical variation for roeks of Classes IV - Y $\ldots \ldots \ldots \ldots \ldots \ldots \ldots . .92$

VIII. Diagram showing distribution of analyses with respect to silica and alkali-silica ratio for 2,000 igneous rocks. . . . . . . 



\title{
LETTER OF TRANSMITTAL.
}

\author{
DEPARTMHNT OF THE INTELIOR, \\ United States Geologicar, Surver, \\ Washington, D. C., May 26, 1903.
}

SrR: I have the honor to transmit herewith a manuscript by Toseph Paxr.... Iddings entitled "Chemical composition of igneous rocks expressed by means oi" diagrams, with reference to rock classification on a quantitative chemico-mineralogical basis," and to recommend that it be published as a Professional Paper.

The significance of a large number of chemical analyses of igneous rocks, with their many variable elements, each having a great range, is somewhat difficult of comprehension to all geologists and petrographers who have not devoted much time to their study. Mr. Iddings presents a method of expressing the most significant factors in each analysis by a simple diugram, and by grouping these individual diagrams on a certain principle he has succeded in representing the great variation in composition and the chemical relationships of almost the entire range of igneous rocks in a way that is comprehensible at a glance. The facts thus brought out are discussed by him in their bearing on petrographic. system. As a successful attempt at the elucidation of a complex problem the paper is of importance to all students of igneous rocks.

Very respectfully,

Whitman Cross, Geologist in Charge, Section of Petrology.

Hon. Charles D. Walcott, Director United States Geological Survoy. 



\title{
CHEMICAL COMPOSITION OF IGNEOUS ROCKS EXPRESSED BY MEANS \\ OF DIAGRAMS, WITH REFERENCE TO ROCK CLASSIFICATION ON A QUANTITATIVE CHEMIC0-MINERALOGICAL BASIS.
}

\author{
By Joseph Paxson Iddings.
}

\section{INTRODUCTION.}

The value of graphical methods for expressing relative quantities has been well established in all kinds of statistical exposition and discussion. Their use in conveying definite conceptions of relative quantities of chemical and mineral components of rocks is becoming more and more frequent, and the value of the results in some cases can not be overestimated. This is especially true when a series or group of rocks is being considered. The intricate variations in the amounts of numerous mineral components, or of chemical components, baffle most attempts to comprehend their interrelationships by simple contemplation or by study of the numbers in which they may be expressed. Many facts and relations are overlooked which are reudily observed when diagrams are used to represent numerical figures. Moreover, visual memory is sufficiently developed in most persons to enable them to carry in mind simple geometrical forms, where it does not permit them to recollect manifold asscmblages of oft-repeated numbers. Mental impressions of simple diagrams are, therefore, more definite and lasting and enable the student to store up a much greater amount of quantitative data than he could otherwise acquire.

Evidence of the appreciation of this fact by petrographers is found in the increasing use of diagrams in petrographical literature, and a brief sketch of the growth and elaboration of graphical methods for presenting quantitative petrographical data will serve as an appropriate introduction to the subject in hand. From this notice will be excluded those diagrams which serve to connect the names of rocks in series after the manner of expressing genealogical relationships, in which diagrams the space relationships are not strictly quantitative. Such diagrams have been used by George H. Williams, H. O. Lang, and other petrographers. 


\section{HISTORICAI, REVIEW.}

Among the earliest diagrams employed to represent relative quantities of rock constituents are those used by Reyer in 1877." They are more or less generalized expressions, in one instance of the varying proportions of the chicf oxide components in rocks and meteorites; in another of the varying amounts of the commoner rock-making minerals capable of being developed in rock magmas with different silica content. The diagrams used are rectangles subdivided by curved lines corresponding to the variations mentioned. Other diagrams express the variations of different kinds of rocks with respect to silica; the relation between specific gravity, silica percentage, and texture of mineralogically similar rocks; and the relative abundance of rocks of different compositions and textures.

Subsequently in his work, Theoretische Geologie, ${ }^{b}$ he reproduces two of these diagrams and introduces others showing the proportions of chemical components in two series of minerals (feldspars and pyroxenes) and others showing the proportions of the chief minerals in three kinds of rocks. One diagram consists of adjacent, long, narrow rectangles, each divided into rectangular parts proportionate to the percentages of the oxide constituents in certain kinds of rocks, from granite to gabbro, and in five types of meteorites.

Judd, ${ }^{c}$ in bis book on Volcanoes, in 1881, makes use of a diagram to represent the varying amounts of chemical elements in rocks and meteorites. It is similar in form to one used by Reyer, already described.

In 1890 the author ${ }^{d}$ constructed diagrams to exhibit the variation in chemical composition of rocks forming geologically related groups or series. In these diagrams the quantitative data represent specific analyses of different rocks in each series. The oxide constituents of each rock are referred to rectangular coordinates, the silica being plotted as abseissas, and the other oxides-alumina, ferric and ferrous iron, magnesia, lime, soda, and potash-being plotted as ordinates. Straight lines connecting successive loci of any one oxide, as those of alumina, indicate the variations in the amount of ench constituent in the rock series. This is the chief feature brought out by these diagrams, the range and character of the variations being shown.

Similar diagrams have been used by Dakyns and Tealle to illustrate the chemical variations in the plutonic rocks of Garabal Hills and Meall Braec,

a Reyer, E., Beitrag zur Fysik der Eruptionen. Vienna, 1877.

$b$ Reyer, E., Theoretische Geologie. Stuttgart, 1888.

c Judd, J. W., Volcanoes. New York, 1881, p. 322, fig. 88.

a Iddings, J. P., The mineral composition and geological occurrence of certain igncous rocks in the Yellowstone National Park: Bull. Philos. Soc. Washington, vol. 11, January, 1890, pp. 207 and 211; also in Twelfth Ann. Rept. U. S. Geol. Survey, 1892, pp. 629 and 649; Mon. U. S. Geol. Survey, vol. 32, pt. 2, pp. 119 and 136; Origin of igneous rocks, Bull. Philos. Soc. Washington, vol. 12, June, 1892, and in Jour. Geol., vol. 1, 1893, p. 173.

e Dakyns, J. R., and Teall, J. J. H., Quart. Jour. Geol. Soc. London, vol. 48, 1892, p. 116. 
Scotland; by Washington to express the chemical variation in the volcanic rocks of Aygina and Methana, Grecian Archipelago, ${ }^{a}$ and in the rocks of Magnet Cove, Arkansas; ${ }^{b}$ and by Cross ${ }^{c}$ to show the chemical composition of the rocks of the Rosita Volcano, Colorado.

Harker ${ }^{\text {ct }}$ has employed the same form of diagram, but.has used atomic ratios of the chemical elements instead of the oxides, to show the variations in the rocks of Carrock Fell, England. Subsequently he modified it slightly by using percentages of the oxide components instead of their molecular proportions to discuss the possibility of distinguishing jgneous rock series from mixed igneous rocks. ${ }^{e}$

Loewinson-Lessing $f^{f}$ has also made use of this method of exhibiting the variability of chemical composition in eruptive rocks, illustrating the great divergence in the composition of rocks classed under one name in the qualitative system and also the differences in composition of average magmas.

In 1900 Pirsson" pointed out the possibility of relating the variations in the chemical composition of rocks of one geological body to their position. in that body by introducing their space relations into this form of diagram. He accomplished it by letting the abscissas represent distances in the rock hody between the various rocks analyzed. By this modification of the diagram just deseribed, he showed that the variations in chemical composition in such cases follow very regular laws, and with one or two elements determined in a variety of the rock the remainder may be found by means of the diagram.

Similar diagrams have since been used by Washington in discussing the differentiation in the rocks of Magnet Cove. ${ }^{h}$

Pirsson has also used a somewhat similar diagram to represent the variations in mineral composition of rocks of Yogo Peak, Little Belt Mountains, Montana. ${ }^{i}$

In 1896 Becke $^{j}$ represented the chemical composition of igneous rocks by diagrams of two kinds, constructed in a manner different from that of those just described. The first is a modification of a three-coordinate diagram for three variables, whose sum is a constant, based upon an equilateral triangle. The three factors chosen are $\mathrm{Ca}, \mathrm{Na}, \mathrm{K}$. The zero point of the coordinate axis is the center of the triangle. From this point three axes run to the corners of the triangle, one for each of the elements, $\mathrm{Ca}, \mathrm{Na}, \mathrm{K}$. The spot within the

\footnotetext{
aWashington, H. S., Jour. Geol., vol. 3, 1895, p. 160.

$b$ Washington, H. S., Bull, Geol. Soc. America, vol. 11, 1900, p. 404.

c Cross, W., Seventeenth Ann. Rept. U. S. Geol. Survey, pt. 2, 1896, p. 324.

$d$ Harker, A., Quart. Jour. Geol. Soc. London, vol. 51, 1895, p. 146.

$e$ Harker, A., Jour. Geol,," vol. 8, 1900, pp. 389-399.

$f$ Loewinson-Lessing, F., Compte rendu Congrès Géol. International, Seventh session, St. Petersbourg, 1899, Pls. I, II, and III.

y Pirsson, L. V., Twentieth Ann. Rept. U.S. Geol. Survey, pt. 3, 1900, p. 569 et seq

$h$ Washington, H. S., Bull. Geol. Soc. America, vol. 11, 1900, p. 651.

iOp. cit., p. 568.

$j$ Becke, F., Min. Pet, Mitth., vol. 16, 1897, p. 315-320
} 
triangle determined by the values of $\mathrm{Ca}, \mathrm{Na}, \mathrm{K}$, in a given rock analysis is the locus of the analysis (Analysenort). The varions positions of these spots indicate the relative amounts of the elements named in the rocks compared. The other chemical elements in the rocks are represented on a second diagram supposed to stand at right angles to the plane of the first. It is drawn with rectangular coordinates, the abcissas being proportional to the ratio $\frac{\mathrm{Ca}}{\mathrm{Ca}+\mathrm{Na}+\mathrm{K}}$, and the ordinates representing the values of $\mathrm{Si}, \mathrm{Al}, \mathrm{Fe}, \mathrm{Mg}$. Each of these elements is located by a spot in the diagram.

In January, 1897, Michel Lévy a devised diagrams to exhibit certain chemicomineralogical characters of igneous rocks, a single diagram representing an individual rock. The component oxides are first distributed into two groups, those that may combine with $\mathrm{Al}_{2} \mathrm{O}_{3}$ to form feldspars and those that may not. All the $\mathrm{Al}_{2} \mathrm{O}_{3}$ is set aside for feldspars, and to it is allotted $\mathrm{K}_{2} \mathrm{O}, \mathrm{Na}_{2} \mathrm{O}$, and sufficient $\mathrm{CaO}$ to satisfy it. An excess of $\mathrm{CaO}$ is placed with iron and magnesia. Silica may be introduced into the diagram or omitted, and in a subsequent publication " in the same year is expressed only in numbers stating the percentage. Rectangular coordinates are used and the values of the components are laid off on two axes intersecting at the zero point. Below this point is plotted the percentage of potash, and also that of iron oxide. To the right of the zero point is plotted the percentage of soda which may combine with alumina. In this direction, also, may be plotted the percentage of silica. Above the zero point is plotted the lime which may combine with alumina, and also the percentage of magnesia. If there is more lime than will. satisfy the alumina remaining after potash and soda have been satisfied, this lime is plotted to the left of the zero point. Triangles are constructed by connecting the end of the potash line with that of the soda line, and the soda with feldspathic lime. Magnesia is connected with nonfeldspathic lime, and this with iron. Two triangles to the right of the $y$ axis give an indication of the feldspathic constituents of the rock. The other two triangles to the left of the $y$ axis represent the nonaluminous constituents. Modifications of the diagrams enter when there is an excess of alumina over alkalies and lime, and again when alumina is less than the alkalies.

Michel Lévy in the publication last cited also employs diagrams showing curves of variation for the percentages of the chemical components when subdivided in the manner just described.

These triangular diagrams have been used by Lacroix in describing the basic rocks accompanying the lherzolites and ophites of the Pyrenees, ${ }^{c}$ and also in his

a Lévy, A. Michel, Bull. Serv. Carte géol. France, vol. 9, No. 57, 1897, p. 38 and Pls. VII, VIII.

$b$ Lévy, A. Michel, Bull. Soc. gêol. France, 3d series, vol. 25,1897 , Pls. X to XV.

c Lacroix, A., Compte rendu Congrès Géol. International, Eighth session, Paris, 1901, Part II, pp. $834,835$. 
work on the alkaline rocks of the petrographical province of Ampasindava, Madagascar. ${ }^{\prime}$

In May, 1897, Brögger ${ }^{b}$ presented a modification of the individual diagrams devised by Michel Lévy. This was done in illustrating the chemical composition of the group of rocks associated with the laurdalite of the Christiania region. Brögger's diagrams are composed of four coordinates in a plane intersecting in a common zero point. One is horizontal, another vertical, and the other two inclined, one $60^{\circ}$ to the right, the other $60^{\circ}$ to the left of the vertical axis. On these axes are plotted the molecular proportions of the chicf oxide components of a rock. On the upper vertical axis are plotted the molecules of $\mathrm{CaO}$, on the lower vertical axis the $\mathrm{Al}_{2} \mathrm{O}_{3}$. On the horizontal axis right and left is plotted the $\mathrm{SiO}_{2}$, half and half. On the upper right inclined axis is plotted $\mathrm{MgO}$; on the upper left one, the $\mathrm{FeO}+\mathrm{Fe}_{2} \mathrm{O}_{3}$, indicated separately. On the lower right inclined axis is plotted $\mathrm{K}_{2} \mathrm{O}$, and on the lower left one $\mathrm{Na}_{2} \mathrm{O}$. The extremities of adjacent axes are connected by lines. These diagrams show at a glance the relative proportions of the molecules of the oxide constituents of each rock. The amount of $\mathrm{CaO}$ capable of being combined with $\mathrm{Al}_{2} \mathrm{O}_{3}$, not combined with $\mathrm{K}_{2} \mathrm{O}$ and $\mathrm{Na}_{2} \mathrm{O}$, can be readily found by measuring the lengths of the several axes. The introduction of the silica and alumina adds to the instructiveness of this form of diagram.

Diagrams of this type have been employed by Hobbs ${ }^{c}$ to express the average composition of groups of rocks classed together under various divisions of the qualitative system in general use at the present time. In these diagrams the inclined axes stand at $45^{\circ}$ to the vertical axis.

Hackman ${ }^{d}$ has made use of both the Becke and the Brögger diagrams to express chemical relations among the rocks associated with ijolite in Kunsamo, Finland.

Rosenbusche uses narrow rectangular diagrams to express the relative proportions of the chemical elements (Netallatome) in two series of 26. rocks; one, a foyaite-theralite series, the other a granitodiorite and gabbro-peridotite series.

In February, 1898, the author $f$ in an essay on rock classification, and later in another essay, made use of diagrams exhibiting the variations of rock analyses with reference to percentage of silica and alkali-silica ratio. These are constructed by using the silica percentages as abscissas, the zero point being to the

a Lacroix, A., Nouv. Arch. Museum Hist. Nat, 4th series, I, Paris, 1902, p. 180 et seq.

b Brögger, W. C., Die Eruptivgesteine des Kristianiagebietes. III. Videnskabsselskabets Schrifter. I. Math.-Naturv. K1. 1897. No. 6. Christiania, 1898.

c Hobbs, W. H., Jour. Geol., vol, 8, 1900, pp. 1-31.

d Hackman, V., Bull. Com. Geol. Finlande, vol. 11, Helsingfors, 1900.

e Rosenbuseh, H., Elemente der Gesteinslehre. Stuttgart, 1898.

$f$ Iddings, J. P., Jour. Geol., vol, 6, 1898, pp. 92-111, 219-237. 
right of the diagram, and 100 to the left. The ordinates-represent the ratios obtained by dividing the sum of the molecular proportions of the alkalies, $\mathrm{K}_{2} \mathrm{O}$ and $\mathrm{Na}_{2} \mathrm{O}$, by that of silica. The loeus of each analysis is indicated by a round spot or by figures of other shapes.

In the same year, 1898, Michel Lévy " presented a new mode of coordination of diagrams to represent rock magmas, based in a measure on those just published by the author, to some features of which Michel Lévy took exception. These diagrams by Michel Lévy give the loci of rock analyses referred to two coordinates in the plane of drawing, and also to a third axis projected on the same plane. The abscissas ( $x$ ) represent the silica percentages in the "white components" (elements blancs) of the rocks compared. The ordinates $(y)$ represent the sum of the percentages of $\mathrm{K}_{2} \mathrm{O}, \mathrm{Na}_{2} \mathrm{O}$, and the $\mathrm{CaO}$ which may enter feldspar combined with $\mathrm{Al}_{2} \mathrm{O}_{3}$. The third coordinates (z) represent the percentages of $\mathrm{MgO}$, $\mathrm{FeO}, \mathrm{Fe}_{2} \mathrm{O}_{3}, \mathrm{CaO}$, and $\mathrm{SiO}_{3}$ in the ferromagnesian components of the rocks. The loci of the analyses are fixed by the rectangular coordinates $x$ and $y$. Their relation to the axis $z$ is indicated by their position with reference to diagonal lines representing the projected traces of certain planes with an inclined plane through 2 .

The usefulness of this diagram, which appears when the compositions of restricted groups of rocks are illustrated, is seriously impaired by repeated overlapping when many kinds of rooks are taken into accoint. The method undertakes to distribute the analyses with respect to the relativo abundance of the white (leucocratic) components-quartz and feldspathic minerals-and the dark (melanocratic) ferromagnesian components in the first instance, and also with respect to the silica content of the leucocratic components.

It is clear that rocks containing various amounts of nephelite, leucite, and anorthite molecules with closely similar percentages of silica will be mingled together in the same part of the diagram, and that rocks with preponderant ferromagnesian minerals, since they are plotted wholly on a basis of characters belonging to the subordinate feldspathic minerals, will be indiscriminately mingled in one portion of the diagram.

In 1899 Loewinson-Lessing, ${ }^{b}$ in his studies of eruptive rocks, an abstract of which was presented, without diagrams, to the International Geological Congress at St. Petersburg in 1897, makes use of two forms of diagrams, one after the method of the author, already alluded to, and another based on the method of relative rectangular areas used by Reyer. The diagram represents the various molecular proportions of the chemical components of a rock, or may represent the average of a group of rocks. It is a rectangular area divided by horizontal 
lines into spaces whose vertical dimensions correspond to the proportions of $\mathrm{SiO}$, $\mathrm{R}_{2} \mathrm{O}_{3}$, RO, and $\mathrm{R}_{2} \mathrm{O}$ in each case. These rectangles are subdivided by vertical lines into spaces. whose horizontal dimensions correspond to the proportions of $\mathrm{Nl}_{2} \mathrm{O}_{3}$ and $\mathrm{Fe}_{2} \mathrm{O}_{3}$ in $\mathrm{R}_{2} \mathrm{O}_{3}$, of $\mathrm{FeO}, \mathrm{CaO}$, and $\mathrm{MgO}$ in $\mathrm{RO}$, and of $\mathrm{K}_{2} \mathrm{O}$ and $\mathrm{Na}_{2} \mathrm{O}$ in $\mathrm{R}_{2} \mathrm{O}$.

In 1900 Mügge" devised a form of diagram to express the proportions of the chemical components of rocks, based upon some features of Michel Lévy's. and Brögger's diagrams. Several modifications of the diagram were suggested by Mügge. It will be sufficient to describe the form used by him to illustrate the composition of a number of selected rocks. The relative quantities of the oxide components, expressed molecularly, are plotted on four coordinate axes in one plane intersecting in a zero point and making equal angles with one another. One is vertical, one horizontal, and two inclined at $45^{\circ}$. The silica is represented by an octagonal area, constructed by plotting one-eighth of the total silica plus and minus on each axis. The other components are plotted outside this area on particular axes by adding their values to the silica already plotted. $K_{2} \mathrm{O}$ is plotted on the upper left inclined axis, $\mathrm{Na}_{2} \mathrm{O}$ on the upper right one, $\mathrm{MgO}$ on the lower left inclined axis, $\mathrm{CaO}$ on the lower right one. $\mathrm{FeO}$ is plotted on the lower rertical axis, followed by $\mathrm{Fe}_{2} \mathrm{O}_{3}$ in the same direction. $\mathrm{Al}_{2} \mathrm{O}_{3}$ is divided into three parts, when sufficient, the first, equal molecularly to $\mathrm{K}_{2} \mathrm{O}$, is plotted to the left on the horizontal axis. A second, equal to the $\mathrm{Na}_{2} \mathrm{O}$ is plotted upward on the vertical axis, and the remainder of the $\mathrm{Al}_{2} \mathrm{O}_{3}$ is plotted to the right on the horizontal axis. When there is less $\mathrm{Al}_{2} \mathrm{O}_{3}$ than $\mathrm{K}_{2} \mathrm{O}+\mathrm{Na} 2 \mathrm{O}$, enough $\mathrm{Fe}_{2} \mathrm{O}_{3}$ is added to $\mathrm{Al}_{2} \mathrm{O}_{3}$ upward on the vertical axis to equal $\mathrm{Na}_{2} \mathrm{O}$. In this manner the relative amounts of potash-alumina molecules, soda-alumina molecules, and lime-alumina molecules are indicated.

In 1900 and 1901 Osann $^{b}$ made use of several types of diagrams to illustrate: chemical characteristics of igneous rocks in his essay on a chemical classification of igneous rocks.

One is a triangular diagram somewhat similar to that used by Becke; exhibiting the distribution of analyses with respect to three coordinate axes making equal angles with one another. The three chemical factors of rocks employed in their comparison are chemico-mineralogical to some extent. One factor (A) consists of the sum of the percentages of $\mathrm{K}_{2} \mathrm{O}$ and $\mathrm{Na}_{2} \mathrm{O}$ which may be combined with $\mathrm{Al}_{2} \mathrm{O}_{3}$ in equal molecular amount. A second factor (C) represents the per(entage of $\mathrm{CaO}$ which may be combined with $\mathrm{Al}_{2} \mathrm{O}_{3}$ in equal molecular amount. The third factor (F) represents the sum of the percentages of the remaining $\mathrm{CaO}$,

a Mügge, O., Neues Jahrb. Min., Geol. ind Pal., 1900, vol. 1, pp. 100-112, Pls. V-VII. b Osann, A., Min. Pet. Mitth., vol. 19, 1900, PIs. IV-VIII, XXV; vol. 26; 1901, Pls. VIII-XIV. 
$\mathrm{MgO}, \mathrm{FeO}$. The sum of these is assumed to be a constant, and their relative proportions are determined on a basis of 20 units. With these three variables the position of a rock analysis within the equilateral triangle is located.

A second form of diagram expresses the variation in silica and alkalinity in certain rocks by means of lines connecting loci of analyses referred to two rectangular coordiuates, the abscissas corresponding to the alkalinity, and the ordinates to the silica percentage. Another diagram of the same type exhibits the variability of different kinds of rock with respect to the relative proportions of soda and potash.

A third form of diagram is composed of narrow rectangles, whose lengths correspond to the range of variation in silica ratios for different kinds of rocks. The rectangles are placed by the side of one another, with their longer diameters adjusted to one ordinate representing values of silica ratios.

In 1902 Reid" used an equilateral triangle whose sides are divided into 100 parts, as a basis on which to plot the oxide components of igneous rocks.

\section{PURPOSE OF THE DIAGRAMS.}

A study of the various diagrams that have been employed by petrographers shows that their value depends upon the readiness with which their significance may be grasped and the use that is made of them. The first condition varies directly as the simplicity of the method of construction, both as to geometrical form and as to the factors on which they are based. The simpler the form of the diagram and the more characteristic its shape, the more definite the visual impression and the stronger the hold on the mind. It is also self-evident that the simpler the factors entering into it the better. The quantitative values of single rock components are simpler conceptions than intricate ratios and functions involving several different rock components. Moreover, a diagram is comparatively worthless unless' it can be made to convey some definite impression not otherwise obtainable, or obtainable only with difficulty. Its omployment should have some specific object. It is hoped that the diagrams presented in this paper will justify themselves and will produce certain definite impressions on the minds of petrographers who may study them.

The author's first purpose in constructing the accompanying diagrams is to express in the simplest graphical manner the chief chemical composition of all kinds of igneous rocks in a form that will permit the student to compare them at a glance and obtain a comprehensive view of the whole range of differences.

The diagrams are intended to exhibit the manifold character of the variations of the chemical components of rocks, and their extent. They are to show the

a Reid, J. A., Bull. Dept. Geol. Univ. California, vol. 3, 1902, p. 18. 
gradual character of the variations; the transitions from rocks of one composition to those of another; and the distribution of the rocks throughout the range of their variations.

They are to demonstrate the absence of clusters of analyses in definite parts of the whole system and the absence of "natural" subdivisions of rocks on a basis of chemical composition.

They are to show the possibility of continuous series of rocks with diverse compositions, and to illustrate the chemical identity of rocks belonging to different genetic families.

Finally the diagrams exhibit the character of the quantitative classification of igneous rocks proposed by Cross, Iddings, Pirsson, and Washington, the range of composition of the five classes, as well as of the orders; rangs, and subrangs.

\section{CONSTRUCTION OF THE, DIAGRAMS.}

As will be seen by looking at the plates, the diagiams are combinations of two kinds already in use. They consist of assemblages of individual rock diagrams, which are modifications of those devised by Michel Lévy and Brögger, the individual diagrams being arranged in a multiple diagram previously used by the author. The first are used to exhibit the chief chemical components of each rock taken into consideration; the socond, to arrange these in an orderly manner for comparison with one another.

The small, individual diagrams are modified forms of those by Brögger. They are planned to exhibit the relative amounts of the chief oxide components of igneous rocks, namely: $\mathrm{SiO}_{2}, \mathrm{Al}_{2} \mathrm{O}_{3}, \mathrm{Fe}_{2} \mathrm{O}_{3}+\mathrm{FeO}, \mathrm{MgO}, \mathrm{CaO}, \mathrm{Na}_{2} \mathrm{O}, \mathrm{K}_{2} \mathrm{O}$, and also $\mathrm{TiO}_{2}$. Other components are usually so slight that they are negligible in diagrams of such small scale. Owing to the fact that the small diagrams are arranged in the large diagram according to the silica, it is not necessary for the purposes of this paper to introduce $\mathrm{SiO}_{2}$ into the construction of the individual diagram. The relative amount of $\mathrm{SiO}_{3}$ is expressed by the position of the small diagram in the large one. This simplities the small diagram.

The chemical components of rocks may be compared with one another as oxides of metals or as metals. As oxides, the form in which they are expressed in chemical analyses of rocks, they may be compared by means of percentage weights or of molecular proportions. As metals they would be compared by molecular or atomic proportions.

The use of oxides instead of metal elements is the more convenient method of comparison. It bears a more obvions and direct relation to the rock analysis and requires less effort to apply. It is as valuable in the discussion of chemicomineralogical relationships as the use of metal elements. It has, therefore, been adopted in constructing these diagrams.

$$
\text { 14474-No. } 18-03-2
$$


The value of basing the comparisons upon molecular proportions instead of percentage weights rests upon the fact that the chemical equivalency of the components is molecular or atomic, and that in this form they may be readily converted into mineral molecules for purposes of discussion and rock classification.

For purposes of comparison in diagrams of this type it is not necessary to reduce the original analysis to a sum of exactly 100 . So far as the small individual diagrams are concerned the amount of change produced in the lengths of the lines would not be appreciable. Further, all elementary lines in one diagram would be affected in like proportion and the shape of the figure would remain unchanged, even could the alteration in the length of the lines be made visible. It would affect to a variable degree the position of the small diagrams within the large one in the direction of the silica abscissa, which will be discussed later.

The small, individual diagrams are constructed to exhibit the molecular proportions of the component oxides: $\mathrm{Al}_{2} \mathrm{O}_{3}, \mathrm{Fe}_{2} \mathrm{O}_{3}+\mathrm{FeO}, \mathrm{MgO}, \mathrm{CaO}, \mathrm{Na}_{2} \mathrm{O}, \mathrm{K}_{2} \mathrm{O}$, and $\mathrm{TiO}_{2}$. These are derived directly from the rock analysis by dividing the percentage weight of each oxide by its molecular weight. 'There is no further' reduction. They are used in the proportions in which they are found in the rock, the assumption being that equal weights of different rocks are being compared.

Following Brögger's method, the several values of these oxides are plotted on coordinate axes in one place, intersecting at a common zero point. But the arrangement is different. For convenience in drawing on square-ruled paper the axes ehosen are vertical, horizontal, and two at $45^{\circ}$ to these. The simple, approximate relation between the sides and hypothenuse of a $45^{\circ}$ triangle, namely, that when the hypothenuse is 10 units the sides are 7 units each (more exactly 7.071) allows the values to be readily plotted by assuming the diagonal of each square of the ruled paper to be 5 and the side $3 \frac{1}{2}$. With larger diagrams and more exact requirements more accurate methods of plotting may be employed.

Since the small diagrams are to be compared by means of the multiple one, the position of the Brögger diagrams is inverted to conform more closely to the manner of constructing the large diagram. In the multiple diagram the more alkalic, and consequently more alkali-feldspathic, mombers of the series of rocks compared occur in the upper part; the less alkalic, and more ferromagnesian, in the lower part. So the alumina and alkalies are placed in the upper part of the individual diagrams, the iron and magnesia in the lower: The mode of construction is as follows: $\mathrm{Al}_{2} \mathrm{O}_{3}$ is plotted upward on the vertical axis; $\mathrm{K}_{2} \mathrm{O}$ upward to the left on one inclined axis; $\mathrm{Na}_{2} \mathrm{O}$ upward' to the right on the other inclined axis; $\mathrm{CaO}$ is plotted downward on the vertical axis; $\mathrm{MgO}$ downward to the right; $\mathrm{FeO}+2 \mathrm{Fe}_{2} \mathrm{O}_{3}$ downward to the left; and $\mathrm{TiO}_{2}$, when present in appreciable amount; is plotted to the left on the horizontal axis. The end of the $\mathrm{Al}_{2} \mathrm{O}_{3}$ ordinate is connected by lines with the ends of the $\mathrm{K}_{2} \mathrm{O}$ and $\mathrm{Na}_{2} \mathrm{O}$ ordinates, 
producing two triangles having a common side- the $\mathrm{Al}_{2} \mathrm{O}_{3}$ ordinate-and one angle the same in each-45. Consequently the areas of the two triangles are proportional to the lengths of the $\mathrm{K}_{2} \mathrm{O}$ and $\mathrm{Na}_{2} \mathrm{O}$ ordinates. The relative impressions produced by these two triangles, colored. green and yellow in the diagrams, are quantitatively the same as the relations between the molecular proportions of $\mathrm{K}_{3} \mathrm{O}$ and $\mathrm{Na}_{2} \mathrm{O}$. in the rosk.

The end of the $\mathrm{CaO}$ ordinate is connected with the ends of the $\mathrm{MgO}$ and $\mathrm{FeO}+2 \mathrm{Fe}_{2} \mathrm{O}_{3}$ ordinate, making two triangles, colored blue and red in the diagrams, whose areas bear the same proportion to one another as the ratio between the molecules of $\mathrm{MgO}$ and the iron converted to $\mathrm{FeO}$.

When $\mathrm{TiO}_{2}$ is represented, the end of its ordinate is connected with that of the $\mathrm{FeO}$ ordinate.

The $\mathrm{FeO}$ and $\mathrm{Fe}_{2} \mathrm{O}_{3}$ are combined chiefly for simplicity, and the $\mathrm{Fe}_{2} \mathrm{O}_{3}$ is reduced to $\mathrm{FeO}$ because there is then a more uniform basis of comparison and a means of avoiding the analytical defects or errors in the determination of the two oxides of iron. The combination prevents a proper conception of the possibilities of mineral molecular combination so far as the iron is concerned, but this is beyond the adaptability of the diagrams in a strict sense.

It is evident that diagrams constructed on the molecular proportions of the oxide components of rocks may be easily converted into others, based on the proportions of the metal elements, by doubling the lengths of the three upper ordinates, $\mathrm{Al}_{2} \mathrm{O}_{3}, \mathrm{~K}_{2} \mathrm{O}, \mathrm{Na}_{2} \mathrm{O}$. The upper half of each diagram would be double its former size, while the angular shape of the whole would remain the same.

'The multiple diagram, by which the small individual ones are arranged for comparison and study, is constructed in the manner already described in the article on rock classification previously cited. It is intended to exhibit the distribution or variation of igneous rock analyses with respect to the silica and alkalies. The reasons for choosing these two factor's are: First, the important rôle played by silica, the most abundant chemical component, and that which is dominant in the development of quartz, orthoclase, albite, leucite, or nephelite, orthorhombic pyroxene or olivine; second, the equally important rôle played by the alkalies in conditioning the character of the feldspathic mineral components of rocks, which are the preponderant ones in the great majority of cases; third, the fact that the other chemical components-lime, magnesia, and iron oxidesustain to a considerable extent a reciprocal relation to those just mentioned. The greater the silica the less the content in lime, magnesia, and iron oxide. The same is true in general for the alkalies and these oxides.

One of the chicf purposes of the diagram is to distribute the rocks in an orderly manner, so that unlike rocks shall be separated from one another as widely as possible and gradations between the extremes shall be made evident. 
The silica is made the abscissa because it is the greater component and has the widest range. It is plotted in percentages instead of molecular proportions because the process is simpler, and as it is not brought into immediate quantitative relations to the linear elements of the individual diagrams there is no occasion for its quantitative linear comparison with them. Moreover, the large diagran could not be constructed on the same scale of units as the individual diagrams. It is therefore simpler and more convenient to use the percentage of silica given in the analyses.

The vertical ordinates represent the ratio between the sum of the molecular proportions of the alkalies $\left(\mathrm{K}_{2} \mathrm{O}+\mathrm{Na}_{2} \mathrm{O}\right)$ and of the silica $-\frac{\left(\mathrm{K}_{2} \mathrm{O}+\mathrm{Na}_{2} \mathrm{O}\right)}{\mathrm{SiO}_{2}}$. This ratio was selected because of the relation between the development of the alkalifeldspathic minerals in highly feldspathic rocks and the ratio between the alkalies and silica in their magmas; the more siliceous ones yielding more orthoclase or albite, the less siliceous ones more leucite or nephelite. It is not a definite relationship in rocks generally, and, as Michel Lévy has pointed out, the character of the diagram would not have been altered materially had the vertical ordinates been made to correspond directly to the sum of the alkalies. In this case the curved lines which represent the loci of pure quartz-albite-nephelite rocks and quartz-orthoclase-leucite rocks would be straight lines instead of hyperbolas, as in the diagrams here used. There would be a slightly different vertical spacing of the analyses on individual diagrams. They would be spread more on the left side of the ordinate of 60 per cent $\mathrm{SiO}_{2}$, and condensed more on the right of it. The multiple diagrams would be more simply constructed. Otherwise the results would be alike.

With diagrams constructed by using ratios between two or more rock components it matters not whether the analyses discussed by it are reduced to anhydrous compounds, or whether their summation is exactly 100 . The ratio hetween the alkalies and silica is not affected by readjusting the analysis. The ordinate of an analysis so located is not changed by readjusting the percentages in the analysis, but the abscissa would be affected by changing the silica percentage. The position of an analysis would be shifted horizontally to the left or right according as the percentage of silica is raised or lowered.

The zero point of each individual diagram is located in the multiple diagram by reference to the silica percentage and the alkali-silica ratio of the analysis it represents. In some instances several individual diagrams bclong so close together that they would overlap, so as to prevent their shapes from being easily seen. One or more of them is moved to the nearest available space in the multiple diagram. From this it happens that the distribution of the analyses in 
the large diagram is not strictly correct in the more crowded portions of it. A more exact representation of the distribution is given in another form of multiple diagrams on a smaller scale, in which round spots take the place of the individual diagrams.

\section{ROCK ANALYSES USED IN CONSTRUCTING DIAGRAMS.}

Having described the methods by which the diagrams are constructed, a more important matter to be considered is the data to be used-the rock analyses to be compared by them.

Analyses have been selected from all sources available, but not all analyses. It is important that they be correctly made and from good material. These conditions, however, can not always be recognized or known without careful study of the publications in which the analyses appear, and not always then. The collection is not above criticism in this respect. However, care was exercised to omit obviously defective analyses, as well as those from plainly declared altered rocks, except in cases where the altered form may be assumed to correspond approximately to the fresh rock, with the addition of water of hydration. For the strongly ferromagnesian rocks such analyses have been taken sometimes in default of better.

The analyses represent as many varieties of igneous rocks as could be found, with as wide a range as possible. Many analyses closely similar to those introduced into the large diagrams have been omitted because there was not space for them. Consequently the diagrams do not show the proper proportions between the commoner and rarer varieties of rocks. This can be shown by plotting the loci of all acceptable analyses by means of spots in a multiple diagram of fairly large scale. This has been done in constructing the diagram on Pl. VIL, which is composed of 2,000 analyses.

It was thought unnecessary to reprint the analyses themselves, for the reason that nearly all of them will be found in the collection of rock analyses made by Dr. Henry S. Washington and published by the United States Geological Survey, ${ }^{c}$ and a large part of them also in Bulletin of the United States Geological Survey No. 168. Many of them will be found in Rosenbusch's Elemente der Gesteinslehre. So that these collections should be used in connection with the diagrams. For this reason reference is given in many cases to these publications, and to others where numerous analyses are assembled, instead of to the original sources, as the more convenient method of finding them.

A list of the analyses used in the diagrams is given. Their positions on the diagrams may be found by means of the silica percentage and alkali-silica 
ratio, which are stated in each case, besides the position given them when placed differently.

They are classified according to the quantitative classification, and the author wishes to express his thanks to Dr. Washington for the labor he has bestowed on the comparison of the list with his collection and for the correction of errors that appeared in the list. $\mathrm{He}$ is indebted to him also for valuable additions to it.

The list is far from complete, and petrographers may add to it and to the diagrams data which should have been included. But it seems as though enough has been introduced to accomplish the purpose for which the diagrams were prepared.

In order to shorten the references in the lists, a number of abbreviations are used which are given alphabetically.

ABBREVIATIONS MMPLOYED IN BIBLTOGRAPIIC REFERENCES.

A. G.................. American Geologist. Minneapolis.

A. J. S................. American Journal of Science. New Haven.

A. W. B .............. Sitzungsberichte der König. Preuss. Akad. der Wissensch. Berlin.

A. W. W . . . . . . . . . . Sitzungsberichte der K. Akad. Wissensch. Wien.

B. C. G. F............. Bulletin de la Commission géologique de Finlande. Helsingfors.

B. G. S. A............ Bulletin of the Geological Society of America. Rochester.

B. M. A ............. Bergens Museums Aarbog.

Bull, $168 \ldots . . . . . . . .$. Bulletin of the U. S. Geological Survey, No. 168. Washington, 1900.

C. G. I. . . . . . . . . . . Congrès géologique. International, Compte Rendu, VIII session. Paris.

E. K . . . . . . . . . . . . . . Die Eruptivgesteine des Kristianiagebietes, W. C. Brögger. Christiania. I, 1894; II, 1895; III, 1898.

G. G ............... Untersuchung einiger Gesteinssuiten gesammelt in Colebes, von P. and $\mathrm{F}$. Sarasin. C. Schmidt, Wiesbaden, 1901.

G. F. S. F . . . . . . . .... Geol. Fören. i Stockholm Förhandl.

I. R. A .............. The igneous rocks of Arkansas: vol. 2 of Ann. Rept. Geol. Surv. Arkansas.

J. G............... The Journal of Geology. Chicago.

O. N........................ et Pearce. Part I. Geneva, 1902.

M. M ..................Materiaux pour la Minéralogie de Madagascar, A. Lacroix. Nouvelles Archives du Muséum. 4th series, Vol. I. Paris, 1902.

N. J ................. Neues Jahrbuch für Mineralogie, Geologie, etc.

Q. J. G. S ............ Quarterly Journal of the Geological Society of London.

Rosenbusch, El......... Rosenbusch. Elemente der Gesteinslehre. Stuttgart, 1898.

R. T . ................ Roth's tables of rock analyses. Beitrïge zur Petrographic der Plutonischen Gesteine. Berlin.

S. M. Q............. School of Mines Quarterly. New York.

T. M. P. M ...........Tschermak's Min. u. Petr. Mitth. Vienna.

W. T. . . . . . . . . . . . Washington's tables of chemical analyses of igneous rocks from 1884 to 1900. Professional Paper U. S. Geological Survey, No. 14, Washington, 1903.

Z. D. g. G ............ Zeitschrift d. Deutschen geol. Gesellschaft. Berlin.

Z. K ................. Zeitschrift für Krystallographie.

\%. P. G ..............Zeitschrift für Praktische Geologie. Berlin.

Fortieth Par...........U. S. Geol. Expl. Fortieth Parallel. Washington. 
CLASSIFIED LIST OF ANALYSES USED IN CONSTRUCTING DIAGRAMS.

CLASS I. PERSALANE.

Order 3. Columbare.

Rang 1. Alaskase.

SUBRING 1. -

(I. $3,1,1$ )

\begin{tabular}{|c|c|c|c|c|}
\hline $\begin{array}{l}\text { Silica } \\
\text { con- } \\
\text { tent. }\end{array}$ & $\begin{array}{l}\text { Alkali-silica } \\
\text { ratio. }\end{array}$ & Author's name. & Locality. & Reterence. \\
\hline 79.25 & 0.057 & Porphyry .......... & 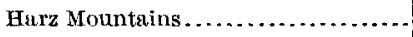 & R. T., 1861,7 . \\
\hline
\end{tabular}

SURRANG 2. MagdebuRgose.

(I. $3,1,2$.

\begin{tabular}{|c|c|c|c|c|}
\hline 79.75 & 0.062 & Quartz-porphyry & Blowing Rock, Watauga County, N. C. & Bull, 168, 52.-W.T... \\
\hline 78.86 & .079 & Felsite-porphyry & Fichtelgebirge, Bavaria..... & R. 'T., 1879, XX. \\
\hline 76.10 & .085 & Granite.... & Crystal Falls district, Mich... & Bull. $168,66 .-\mathrm{W} . \mathrm{T}$. \\
\hline 75.39 & .080 & Khyolite... & Silver Cliff, Colo............. & Bull. 168, 149.-W. T. \\
\hline 74.40 & .079 & Granite.......... & Pikes Peak district, Colo..... & Bull. 168, 142.-W. T. \\
\hline
\end{tabular}

Subrañg 3. Alaskose.

(I. 3, 1, 3.)

\begin{tabular}{|c|c|c|c|c|}
\hline 80.24 & 0.070 & Granite. & Wexford, Ireland.. & R. T., 1861-2, \\
\hline 79.57 & .070 & Rhyolite. & Montgomery County, N.C...... & Bull. 168, 53.-W. T. \\
\hline 77.68 & .077 & Granitite & Eldorado County, Cal. & Bull. 168, 199.-W. T. \\
\hline 77.68 & $.075(77.6)$ & Quartz-porphyry & Tryberg Fall, Black Forest..... & N. J., 1888, II, 29.一W. 'T'. \\
\hline 77.59 & .059 & Rhyolite....... & Hauraki Goldfield, New Zealand.. & Q.J. G. S., 1899, LV, 467.-W. T' \\
\hline 77.83 & $.079(.078)$ & Alaskite ........... & Tordrillo Mountains, Alaska .... & Bull. 168, 228.-W. T. \\
\hline 77.20 & .080 & Qunrtz-porphyry & Kroftkollen, Norway ... & Z, K., XVI, 77.-W. T. \\
\hline 77.05 & .080 & Aplite ........... & Butte, Mont ........... & Bull. 168, 118.-W. T. \\
\hline 77.03 & $.081(76.8-.082)$ & Granitite. & Pikes Peak district, Colo. & Bull. 168, 142.-W. T. \\
\hline 77.02 & $.077(77.1)$ & Granite........ & Platte Canyon, Colo ......... & Hull. 168, 164.-W. T. \\
\hline 76.87 & .078 & Aplite.. & Butte, Mont ......... & Bull. 168, 118.-W. T. \\
\hline 76.73 & $.077(76.6)$ & Aplitic granophyre. & Hennum, Norway ........ & Z. K., XVI, 77.--W.T. \\
\hline 76.10 & .063 & Mnscovite-granite. & Omeo, Victoria..... & Trans. R. S. Vict-W. T. \\
\hline 75.84 & .081 & 'Tordrillite...... & Tordrillo Mountains, Alaska & Bull. $168,229 .-W . T$. \\
\hline 75.52 & $.080(75.7)$ & Obsidian ... & Obsidian Cliff, Yellowstone Park. & Bull. 168, J04.-W. T. \\
\hline 74.70 & .083 & $\ldots$ do $\ldots .$. & .... do ................... & Do. \\
\hline 74.24 & .069 & Rhyolite... & Plumas County, $\mathrm{Cal}$. & Bull. 168, 178.-W. T. \\
\hline 73.11 & $.082(73,0)$ & Pitchstonc........... & 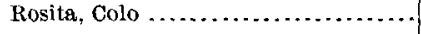 & Bill. 168, 150.-W. T. \\
\hline
\end{tabular}

SUBRANG 4, -

(I. $3,1,4$.

\begin{tabular}{|c|c|c|c|c|}
\hline 83.08 & 0.054 & Spherulitic rock. & Wales... & Tenll, British Petrography, 389 . \\
\hline 82.80 & .049 & Perlite.. & Monte Menone ........... & R. T, 1869, XC. \\
\hline 76.00 & .067 & Granite.. & Plumas County, Cal ....... & Bull, 168, 18\%.-W.T. \\
\hline 75.19 & $.076(75.1)$ & Rhyolite......... & Madison Plateau, Yellowstone Park.. & Bull. 168, 105.-W. T. \\
\hline
\end{tabular}


Classified list of analyses used in constructing diagrams-Continued.

SuRrang 5. Westphatose.

(I. $3,1,5$.

\begin{tabular}{|c|c|c|c|c|}
\hline $\begin{array}{l}\text { Silien } \\
\text { eon- } \\
\text { tent. }\end{array}$ & $\begin{array}{c}\text { Alkali-siliea } \\
\text { ritio. }\end{array}$ & Author's name. & Locality. & Reference. \\
\hline 83.57 & 0.053 & Quartz-keratophyre.. & Westphalia ......... & N. J., 1893, VIII, 616.-W.'T. \\
\hline .0 .42 & .059 & $\ldots$. do $\ldots . . . . . . . .$. & $\ldots \ldots$ do $\ldots \ldots \ldots \ldots \ldots \ldots \ldots \ldots \ldots \ldots \ldots \ldots \ldots \ldots$ & N. J., 1893, VIII, 632,-W.T. \\
\hline 77.32 & $.081(.083)$ & Granito. & Rorldö, Sweden.. . & W. T. \\
\hline
\end{tabular}

Rang 2. Alskachase.

Subrang 2. Mithalose.

(I. $3,2,2$.)

\begin{tabular}{|c|c|c|c|c|}
\hline 73.15 & 0.055 & Rhyolite.. & Nagy-Mihaly, Hungary ............. & W. T. \\
\hline 73.07 & $: 074$ & 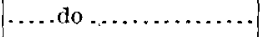 & Washoe, Ner ...................... & Bull. 168, 174.--W. I. \\
\hline 72.68 & .054 & ..... do $\ldots . . . . . . . . . . . .$. & Nagy-Mihaly, Hungary .............. & W. T. \\
\hline 70.59 & .057 & ..... do $\ldots$ & $\ldots$. do $\ldots . . . . . . .$. & Do. \\
\hline
\end{tabular}

Subrang 3. Tehamose.

(I. $3,2,3$.

\begin{tabular}{|c|c|c|c|c|}
\hline 79.95 & 0.049 & Folsite-porphyry ..... & Bohemia & R. T., 1884, XVIII. \\
\hline 77.34 & .069 & Aplito ......... & Kìrnechthąl, Vosges . . . . . . . . . . . & Rosenbusch, El., 207. \\
\hline 76.12 & $.0 \overline{9} 9$ & Granitite. & Riesengebirge, Silesia. . & I. J., 1898, XII, 233.-W. T. \\
\hline 75.89 & $.069(75.8)$ & Rhyolite.. & Mount Sheridan, Yellowstone Park.. & Bull. 168, 105.-W. T. \\
\hline 75.34 & .070 & ......do... & Elephants Back, Yellowstone Park & Do. \\
\hline 75.21 & .072 & Granitite ..... & Riesengebirge, Silesia ........... & N. J., 1898, XII, 233.-W. T. \\
\hline 74.65 & .074 & Rhyolite. & Tehama County, Cal . & Bull. 168, 178.-W. 'T. \\
\hline 74.41 & $: 067$ & Granite... & Riesengebirge, silesia... & N. J., 1898, XII, 233.-W. T. \\
\hline 74.34 & .072 & Rhyolite.. & Butte district, Mont.... & Bull. 168, 119.-W. 'T. \\
\hline 73.62 & $.079(78.7)$ & ....do.......... & Tehama County, Cal ........... & Bu1l. 168, 178.-W. T. \\
\hline 72.37 & .058 & Granitite.... & Riesengebirge, Silesia..... & N. J., 1898, XII, 232.-W. T. \\
\hline 72.15 & .067 & .... do... & ..... do ............ & Do. \\
\hline 72.04 & .058 & $\ldots$ do .... & f....do $\ldots \ldots \ldots \ldots \ldots \ldots$ & Do. \\
\hline 72.01 & .073 & Quartz-porphyry ..... & Euroka, Nev.............. & U. S. G. S., Mon. XX, 228. \\
\hline 71.45 & .054 & Granitite ............. & 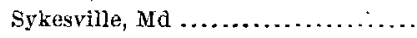 & Bull. 168, 47.-W. T. \\
\hline
\end{tabular}

SUBRANG 4. ALSBACHOSE.

(I. $3,2,4$.)

\begin{tabular}{|c|c|c|c|c|}
\hline 78.75 & 0.052 & Granite.. & Storgord, Finland.. & R. T., 1869, XL. \\
\hline 77.35 & .052 & i....do..... & Riesengebirge, silesia :........... & N. J., 1898, XII, 233.-W. T. \\
\hline 74.65 & .064 & i Granitite. & .....do... & Do. \\
\hline 74.13 & .077 & Alsbachite. & Melibocus, Odenwald.. & Rosenbusch, Fl, 207,-W. 'I. \\
\hline 73.08 & $.080(72.9)$ & Rhyolite.. & Waihi, Fauraki, New Zealand ....... & Q. J. G. S., 1899, LV, 467.-W. T. \\
\hline 72.89 & .056 & Granitite............. & Riesengebirge, Silesia .............. & N. J., I898, XII, 232.-W. T. \\
\hline
\end{tabular}

SURRATG 5. YUKONOSE.

(I. 3, 2, 6.)

\begin{tabular}{l|l|l|l|l|}
\hline 74.79 & 0.067 & Tonalite-nplite ....... & Yukon River, Alaska............... Bull, 168, 229. -W. T. \\
\hline
\end{tabular}


Classified list of analyses used in constructing diagrams-Continued.

\section{Rang 3. Riesenase.}

SUBRANG 2. -

(I. $3,3,2$.)

\begin{tabular}{|c|c|c|}
\hline $\begin{array}{l}\text { Silica } \\
\text { eon- } \\
\text { tent. }\end{array}$ & $\begin{array}{l}\text { Alkali-silica } \\
\text { ratio. }\end{array}$ & Anthor's name. \\
\hline 72.11 & 0.041 & "Sohliere" in gran \\
\hline 71.14 & .055 & Toscanite.... \\
\hline 66.86 & .052 & Grunite-porphyry \\
\hline
\end{tabular}

\begin{tabular}{|c|c|}
\hline Iocality. & Reference. \\
\hline $\begin{array}{l}\text { Kiesengebirge, Silesia } \ldots \ldots \ldots \ldots \ldots \ldots \\
\text { Rocca Strada; Italy .................... } \\
\text { Nieder Modau, Hesse................ }\end{array}$ & $\begin{array}{l}\text { N. J., 1898, XII, } 235 .- \text { W. T. } \\
\text { J. G., V, 363.-W. T. } \\
\text { W. T. }\end{array}$ \\
\hline
\end{tabular}

Subrang 3. Riesenose.

(I. $3,3,3$.)

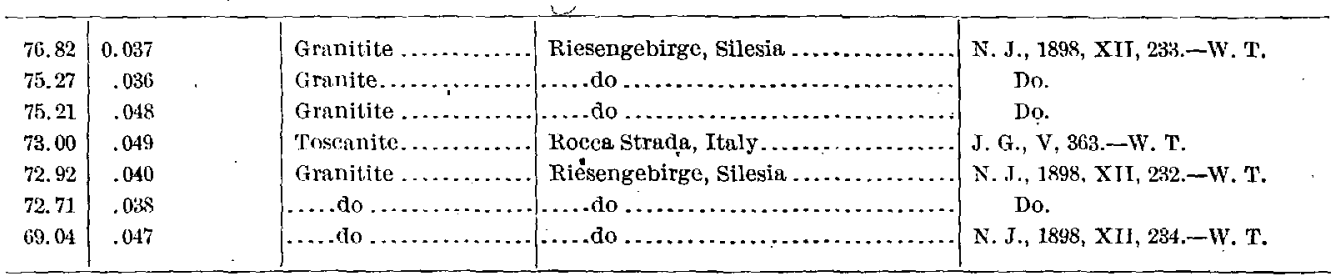

Subrang 4. -

(I. $3,3,4$.

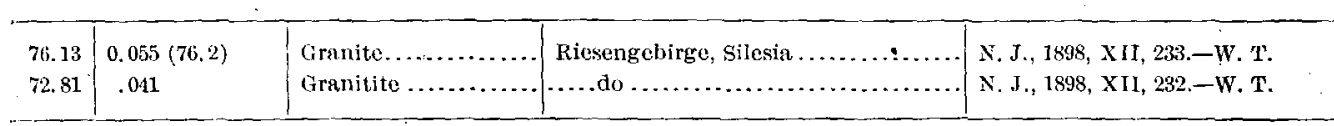

Subrang 5. Vulcanose.

(I. 3, 3, 5.)

\begin{tabular}{|c|c|c|c|c|}
\hline 72.24 & 0.062 & $\begin{array}{l}\text { Quartz-diorite - por- } \\
\text { phyry. }\end{array}$ & Calaveras County, Cal..... & Bull. 168, 203.-W. T. \\
\hline 66.99 & .051 & vulcanite ............ & Vulcano, Iipari ....... & B. G.S. A., V, 601.-W. I. \\
\hline
\end{tabular}

RANG 4 .

SUBliakg 2. -

(I. $3,4,2$.)

\begin{tabular}{l|l|l|l|l}
\hline 68.87 & 0.025 & "Sthlicre" in granite & Riesengebirge, Silesic .............. N. J., 1898, XII, 285.-W. T.
\end{tabular}


Classified list of analyses used in constructing diagrams-Continued.

\section{ORDFir 4. Britannare.}

Rang 1. Liparase.

Subrang 1. Jebachose.

(I. 4, 1, 1.)

\begin{tabular}{|c|c|c|c|c|}
\hline $\begin{array}{l}\text { Silica } \\
\text { con- } \\
\text { tent. }\end{array}$ & $\begin{array}{l}\text { AJkali-silica } \\
\text { ratio. }\end{array}$ & Author's name. & Locality. & Reference. \\
\hline 69.06 & $0.117(69.3)$ & Quartz-orthoclasite.. & Thüringerwald, Prussia............ & W. T. \\
\hline 68.13 & .107 & Quartz-porphyry ..... & Hummelberg, Prussia.................. & Do. \\
\hline
\end{tabular}

SUBrang 2. OMEOSE.

(I. 4, 1, 2.)

\begin{tabular}{|c|c|c|c|c|}
\hline 75.20 & 0.096 & Rhyolite & Silver Clifi, Colo. & Bull, 168, 149.-W. T. \\
\hline 75.07 & .084 & ..... do... & Pine Nut Canyon, Nev ........ & Fortieth Par., I, 652. \\
\hline 74.62 & .095 & .....do. & Humboldt Sink Group, Nev..... & Do. \\
\hline 74.24 & .089 & Rapakiwi. & 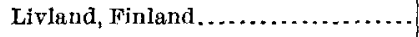 & R. T., $1873, \mathrm{X}$. \\
\hline 71.09 & .112 & Aplite.... & Satteljoch; Predazzo, Tyrol .... & K. A. W. W., CXI, I, 266 . \\
\hline 70.91 & $\cdot 121$ & Graphic granite. & Omeo, Victoria................. & Trans. R. S. Viet:-W.T. \\
\hline 68.99 & .115 & Pitehstone ... & Ponza, Italy $\ldots \ldots \ldots$ & R. T., 1879, LII. \\
\hline 68.87 & .084 & Granite...... & Omeo, Victoria...... & Trans. R. S. Vict.-W.T. \\
\hline
\end{tabular}

Subrang 3. Liparose.

(I. $4,1,3$.)

\begin{tabular}{|c|c|c|c|c|}
\hline 77,61 & 0.088 & Granite. & Cape Ann, Mass.. & J. G. VI, 793.-W. T. \\
\hline 76.49 & .092 & Paisanite. & Magnolia, Mass.... & J. G. VII, 113.-W. T. \\
\hline 76.33 & .092 & Graphic granite & Hochland ............. & R. T., 1869, ХLII. \\
\hline 76.30 & .088 & Alaskite.... & Alaska .................... & Bull. $168,228 .-W$. T'. \\
\hline 76.20 & .091 & Obsidian .. & Obsidian Hill, N. Mex... & Bull, 168, 172.-W. T. \\
\hline 76.05 & .092 & Aplitic granophyre. & Konerudwege, Norway........ & z. K., XVI, 77.-W, 'T. \\
\hline 75.71 & $.094(75.6)$ & Rhyolite. & Great Paint Pots, Yellowstone Purk & Bull. 168, 105.-W. T. \\
\hline 75.41 & .094 & Comendite.. & Iskagan Bay, Siberia ........... & A. J. S., XIT, 1902, 180.-W.T. \\
\hline 75.44 & .089 & Rhyolite. & Fish Creek Mountains, Nov . & Fortieth Par., I, 652. \\
\hline 75.30 & .082 & .....do ...... & Rimini, Mont ................ & Bull. 168, 119;-T. T. \\
\hline 75.17 & .085 & Granitite. & Pikes Peak distriet, Colo.... & Bull. 168, 142.-W. T. \\
\hline 74.90 & 04 & Rhyolite.. & Castle Mountain district, Mont & Bull. I68, 129.-W. T. \\
\hline 74.87 & .084 & Granite......... & Brookville, Ma....... & Bull. I68, 46.-W.T. \\
\hline 74.76 & .098 & Comendite ..... & Comende, San Pietro, Sardinia. & Rosenbusch, El., 257.-W.T. \\
\hline 74.45 & .090 & Rhyolite... & Chalk Mountain, Colo ....... & Bull. 168, 1566.-W. T. \\
\hline 74.05 & .096 & Obsidian ... & Mono Craters, Cal............ & Bull. 168, 219.-W. 1. \\
\hline 73.93 & .100 & Granite... & Quincy, M̀ass............... & J. G., VI, 799.-W. T. \\
\hline 73. 69 & .100 & Granite-porphyry & 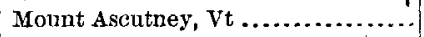 & Bull. 168, 25.-W. 'T. \\
\hline 73.51 & $.094(73.4)$ & Granitite... & Pikes Peak district, Colo... & Bull. 168, 142.-W. T. \\
\hline 73,35 & .106 & Paisanite...... & Mosques Canyon, Tex........ & T. M. P. M., XV, 439.-W. T. \\
\hline 73.09 & .085 & Granite-porphyry.. & Albany, N. H......... & A. J. S., XX, 1881, 25. \\
\hline 73.03 & .104 & Syenite-porphyry.. & Mount Ascutney, Vt.......... & Bull. 168, 24.-W. T. \\
\hline 72.88 & .093 & Aplite-granite... & Castle Mountain district, Mont. & Bull. 168, 129.-W. T. \\
\hline 72.48 & .099 & Granite.. & .....do....... & Do. \\
\hline 72.35 & .098 & .....do.... & Ironton, Mo .......... & Bull. 168, 60.-W. T. \\
\hline 72.26 & .101 & ......do ... & Albany, N. H... & A. J. S., XXI, 1881, 25. \\
\hline 72.06 & .107 & .....do.... & Stony Creek, Coun .... & B. G. S. A., X, 375.-W.T. \\
\hline
\end{tabular}


ROCK ANALYSES USFD IN CONSTRUCTING DIAGTAMS.

Classified list of analyses used in constructing diagrams-Continued.

\begin{tabular}{|c|c|c|c|c|}
\hline $\begin{array}{l}\text { Siliea } \\
\text { con- } \\
\text { tent. }\end{array}$ & $\begin{array}{l}\text { Alkali-silica } \\
\text { ratio. }\end{array}$ & Author's name. & Locality. & Reference. \\
\hline 71.90 & .102 & Granitite.. & Mount Ascutney, Vt. . & Bull. 168, 24.-W. T. \\
\hline 71,88 & .095 & Porphyry...... & Ironton, Mo................. & Bul1. $168,60 .-$ W. T. \\
\hline 71.67 & .101 & Quartz-porphyry .... & Castle Mountain district, Mont & Bull. 168, 129:-W. T. \\
\hline 71.56 & .086 & Pitchstone........... & Silver Cliff, Colo............ & Bull. 168, 150.-W. T. \\
\hline 71.49 & .109 & Quartz-porphyry. & Gislerud, Norway ... & Z. K, XVI, 46,-W.T. \\
\hline 71.07 & .087 & Granite-porphyry .... & Albany, N. H................ & A. J.S., XXI, 1881, 25. \\
\hline 70.92 & $.096(70.7)$ & Rhyolite ........ & $\begin{array}{l}\text { Upper Geyser Basin, Yellowstone } \\
\text { Park. }\end{array}$ & Bull. 168, 104.-W. T. \\
\hline 70.23 & .113 & Keratophyre ......... & Marblehead Neck, Mass... & Bull. 168, 34-W. T. \\
\hline 70.09 & .092 & Orthoclase-porphyry. & Cornon, Tyrol ............. & R. T., 1879, XXVIII. \\
\hline 70.01 & .118 & Obsidian...$\ldots \ldots \ldots$ & Palmerola, Italy .............. & R. T., 1879, LII. \\
\hline 69.91 & .119 & Egirite-granite ...... & Miask, Ural Mountains........ & A. J. S., XIII, 1902, 180.-W. T. \\
\hline 69.89 & .098 & Rhyolite .......... & Nathrop, Colo ............ & Bull. 168, 164.-W. T. \\
\hline 68.95 & .144 & Grorudite ............ & W. Aker, Norway ................... & E. K., I, 199.-W. T. \\
\hline 68.71 & .114 & Quartz-pantellerite.. & Vieja Mountains, San Carlos, Tex.... & Bull. $168,61 .-\mathrm{W} . \mathrm{T}$. \\
\hline 68.65 & $.110(68.5)$ & $\begin{array}{l}\text { Granite-syenite-por- } \\
\text { phyry. }\end{array}$ & Little Rocky Mountains, Mont.. & Bull. 168, 134.-W. T. \\
\hline 68.34 & .129 & Quartz-syenite... & Bearpaw Mountains, Mont. . & Bull. $68,135 .-W . T$. \\
\hline 66.40 & $.097(66.7)$ & $\begin{array}{l}\text { Quartz - syenite - por- } \\
\text { phyry. }\end{array}$ & Fjelebua, Norway $\ldots . . . \ldots$. & Z. K., XVI, 46.-W. T. \\
\hline
\end{tabular}

SUBRANG 4. KaLteEUDOSE.

$$
\text { (I. } 4,1,4 .)
$$

\begin{tabular}{|c|c|c|c|c|}
\hline 75.92 & $0.093(75.8-.094)$ & Granitite.............. & Pikes Peak district, Colo ............ & Bull. 168, 142.-W. T. \\
\hline 73.61 & .089 & Rhyolite ............ & Oyacachi, South America ........ & R. T., 1879, SIV. \\
\hline 73.50 & $.095(73.8)$ & $\begin{array}{l}\text { Quartz-bearing bos- } \\
\text { tonite. }\end{array}$ & Ampangarinana, Madagasear... & M. M., 55 . \\
\hline 72.56 & $.099(72.8)$ & Rhyolitic pitchstone. & Castle Mountain distriat, Mont ...... & Bull. 168, 129.-W.T. \\
\hline 72,46 & .095 & Rhyolite............. & Guomani, Ecuador, Sonth America... & R. T., 1879, LIV. \\
\hline 71.65 & .119 & Sodu granite... & Hougnatten, Norway................ & E. K., I, 198.-W. T. \\
\hline
\end{tabular}

SUBHAXg 5. NOYANGOSE.

(I. $4,1,5$.

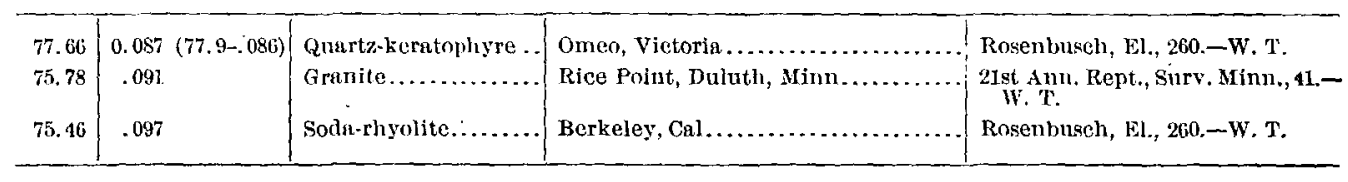

Rang 2. Toscanase.

Subrang 2. Deimenese.

(I. $4,2,2$.)

\begin{tabular}{|c|c|c|c|c|}
\hline 69.19 & 0.102 & Granite. & Tryberg Fall, Schwarzwald ..... & N. J., 1883, II. \\
\hline 68.36 & .078 & Deilenite ............ & Dellen, Helsingland, Sweden . & J. K., II, 59.-W.T. \\
\hline 66.24 & .093 & Toscanite. . :.. & Monte Cueco, Italy ........... & J. G., V, $362,-$ W. T. \\
\hline 65.19 & .093 & ..... do. & Tolfa, Italy........... & Do. \\
\hline
\end{tabular}


Classified list of analyses used in comstructing diagrams-Continued.

Subrang 3. Toscanose.

(I. $4,2,3$.

\begin{tabular}{|c|c|c|c|c|}
\hline $\begin{array}{l}\text { Silica } \\
\text { con- } \\
\text { tent. }\end{array}$ & $\begin{array}{l}\text { Alkali-silica } \\
\text { ratio. }\end{array}$ & Author's name. & Locality. & Reference. \\
\hline 76.48 & 0.087 & Aplite.. & Dargo, Victorin.. & Trans, R. S. Viet.-W. 'T. \\
\hline 76.03 & .081 & .... do ................ & Siema County, Cal...... & Bull. 168, 192.--W. T. \\
\hline 76.00 & .087 & Granodiorite-aplite. & Downieville arca, Cal.. & J. G., VII, 160.-W. T. \\
\hline 75.97 & $.079(76.1-078)$ & Aplite ............ & Sierra County, Cal...... & Bull. $168,192 .-W$. T. \\
\hline 75.74 & .086 & Granite. & Lier, Norway ................. & Z. K., XVI, 77.-W.T. \\
\hline 75.08 & $.084(75.0)$ & Obsidian & Lagunn de Maricunga, Chile. . & Z. D. J., G. LI, 4. \\
\hline 75.01 & $.086(75.1-.088)$ & Alaskite... & 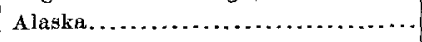 & Bull. $168,228 .-W . T$. \\
\hline 74.95 & .088 & Rhyolite.. & Montezuma Mountains, Nev . & Fortieth Par., I, 652. \\
\hline 74.60 & .081 & $\ldots$....do ..... & Shasta County, Cal........... & Bull. 168, 178.-W. T. \\
\hline 74.49 & $.088(74.6)$ & $\ldots$. do ..... & Thomas Range, Utah........ & Bull. 168, 168.-W. T. \\
\hline 74.37 & .085 & Granitite ...... & Crazy Mountains, Mont ..... & Bull. 168, 120.-W.T. \\
\hline 73.84 & .083 & Rhyolite-perlite. & Mridway Basin, Yellowstone Park.. & Bull. $168,108,-$ W. T. \\
\hline 78.70 & .092 & Granite.......... & Peterhead, scotland................ & R. T., 1884, XIV. \\
\hline 73.64 & .083 & Rhyolite.......... & Lassen trail, Tehama County, Cal.. & Bull. $168,178 .-W$. T. \\
\hline 73.51 & .097 & Obsidian & Modoc County, Cal......... & Bull. 168, 217.-W.T. \\
\hline 73.38 & .080 & Granite... & Lake Raslangen, Sweden .. & W.T. \\
\hline 73.12 & .087 & Quartz-porphyry & Little Belt Mountains, Mont & Bull. 168, 125.-W.T. \\
\hline 72.57 & .090 & Granite........... & 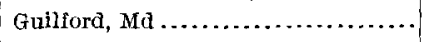 & Bull. $168,46 .-W . T$. \\
\hline 72.40 & .086 & Rhyolite. . & Mount Stover, Cal......... & Bull. $168,178 .-$ W.T. \\
\hline 71.85 & $.087(72.0)$ & $\ldots$.... do ....... & Tower Creek, Yellowstone Purk & Bull. $168,104 .-W . T$. \\
\hline 71.79 & $.082(71.7)$ & Granitite... & Woodstock, Md......... & Bull. 168, 46.-W.T. \\
\hline 71.78 & .085 & Granite........... & Cottonwood Canyon, Utah ............ & Fortieth Par., I, 110. \\
\hline 71.53 & .070 & Granitite.. & Riesengebirge, Silesia ......... & N. J., 1898, XII, 232.-W.T. \\
\hline 71.12 & .098 & Rhyolite.......... & Ponza, Italy .................... & R. T., 1879, LII. \\
\hline 71.08 & $.084(71: 0)$ & Granodiorite ... & El Capitan, Yosemite, Cal..... & Bull. 168, 207.-W.T. \\
\hline 70.87 & .092 & Rhyolite............. & Pennsylvania Hill, silver cliff, Colo & Bull. 168, 149.-W. T. \\
\hline 70.75 & $.080^{\circ}$ & Granite........... & Amador County, CaI ........... & Bull. 168, 200.-W.T. \\
\hline 70.56 & $.105(70.7)$ & Rhyolite...... & Ponza, Italy $\ldots \ldots \ldots$ & \\
\hline 70.54 & .107 & Egirite-grunite .... & Löken, Norway .............. & Z. K., XVI, 57.-W.T. \\
\hline 70.45 & .084 & Granite.......... & Dorseys R11n, Md........... & Bull. $168,47,-\mathrm{W} . \mathrm{T}$. \\
\hline 70.29 & .101 & Rhyolite........ & Harlequin Canyon, Nev...... & Fortieth Yar., I, 652. \\
\hline 69.96 & .089 & Dacite............... & Washoe, Nev ............ & Bull. 168, 174.-W. T. \\
\hline 69.95 & $.098(70.1)$ & Quartz-porphyry ... & Butte district, Mont........ & Rull. $168,119 .-W . T$. \\
\hline 69.94 & .092 & Granite........ & Ironton, $\mathrm{No}$................ & Bull, 168,60.-W. T. \\
\hline 69.68 & .087 & Granite-porphyry & Little Belt Mountains, Mont ... & Bull. 168, 125.-W. T. \\
\hline 69.52 & .108 & Trachyte ......... & Pikes Peak district, Colo.... & Bull. 168, 145.-W. T. \\
\hline 69.45 & .097 & Rhyolo-trachyte..... & Sunset Peak, Mont ............ & Bull. 168, 106.-W. T. \\
\hline 68.88 & .100 & $\begin{array}{l}\text { Quartz-syenite-por- } \\
\text { phyry. }\end{array}$ & Squam Light, Mass....... & J. G., VII, $109 .-W . T$. \\
\hline 68.68 & .077 & Granite........... & Eureka, Nev........ & U. S. G. S., Mon. XX, 228. \\
\hline 68.60 & $.107(68.7)$ & Granite-porphyry & Little Belt Mountains, Mont. & Bull. 168, 125.-W. T. \\
\hline 68.58 & .100 & Granite..... & West Humboldt Mountains, Nev..... & Fortieth Par., I, 110. \\
\hline 68.55 & .095 & $\ldots$... do ... & Shap, England ..... & Q. J. G. S., 47,276 \\
\hline 68.42 & $.083(68.2)$ & Quartz-monzonite. & 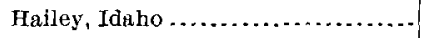 & Bull, 168, 137.-W. T. \\
\hline 68.40 & $.112(68.2)$ & Granite.: & Millstone Point, Conn... & B. G. S. A., X, 375.-W. T. \\
\hline 68.36 & .105 & Nordmarkite.... & Gloucester, Mass............. & J. G., VI, 800.-W. T. \\
\hline 67.45 & .084 & Granodiorite .... & Eldorado Canyon, Cal....... & Bull. 168, 199.-W. T. \\
\hline 67.44 & .104 & $\begin{array}{l}\text { Granite-syenite-p or- } \\
\text { phyry. }\end{array}$ & Little Belt Mountains, Mont ... & Bull. 168, 127.-W. T. \\
\hline 67.12 & .082 & Granite........ & Boulder, Mont... & Bull. 168, 118.-W.T. \\
\hline 67.04 & .107 & $\begin{array}{l}\text { Quartz-syenite-por - } \\
\text { phyry. }\end{array}$ & Little Belt Mountains, Mont & Bull. 168, 127.-W. T. \\
\hline 66.60 & .111 & Akerite............... & Gloucester, Mass. . & J. G., VI, 798.-W. T. \\
\hline
\end{tabular}


Classified list of analyses used in constructing diagrams-Continued.

Surrang 3. Toscanose-Continued.

\begin{tabular}{|c|c|c|c|c|}
\hline $\begin{array}{l}\text { Silica } \\
\text { con- } \\
\text { tent. }\end{array}$ & $\begin{array}{c}\text { Alkali-siljea } \\
\text { ratio. }\end{array}$ & Author's naine. & Locality. & Reference. \\
\hline 66.12 & $.123(66.3)$ & Trachyte. & Wicher Mountain, Colo ... & Bull. 168, 145:-W. T. \\
\hline 65.58 & .094 & ....do do.... & Monte Amiata, Italy ........... & Rosenbusch, El, 269.-W. T. \\
\hline 65.32 & .096 & .....do. & $\ldots$ do $\ldots . . . . .$. & Do. \\
\hline 65.05 & .092 & Toseanite... & $\ldots .$. do $\ldots . . . . . . . . . . . . . . .$. & J. G., V, 362.-W. T. \\
\hline 64.76 & .094 & Trachyte. & .... do .... & Rosenbusch, E1, 269.-W. T. \\
\hline 64.64 & $.114(64.5)$ & Syenite .... & Little Belt Mountains, Mront .... & Bull: $168,125 .-W$. T. \\
\hline $64: 62$ & .118 & Diorite ...... & Mount Ascutney, Vt. ........ & Bull. $168,25 .-\mathrm{W} . \mathrm{T}$. \\
\hline 64.57 & .095 & Toscanite............ & 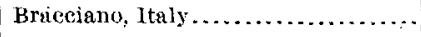 & J. G., V, 362.-W. T. \\
\hline 64.49 & .108 & Porphyrite .......... & 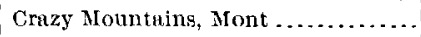 & Bull. 168, 120.-W. T. \\
\hline 62.33 & .110 & Latite .......... & $\begin{array}{l}\text { Clover Meadow, Tuolumne County, } \\
\text { Cal. }\end{array}$ & Bull. 168, 205.-W. T. \\
\hline
\end{tabular}

Subrang 4. Lassenose.

$$
\text { (I. } 4,2,4 .)
$$

\begin{tabular}{|c|c|c|c|c|}
\hline 75.50 & 0.083 & Rhiyolite ........... & Obsidian Cliff, Yellowstone Park & Bull. 168, 104.-W.T. \\
\hline 73.27 & .077 & Granite. & Florence, Mass ... & Bull. 168, 30.-W. T. \\
\hline 73.00 & .075 & ..... do... & Rocklin, Cal............... & Bull. 168, 198.-W.T. \\
\hline 72.59 & .083 & Obsidian & Willow Park, Yellowstone Park. & Bull. 168, 104.-W.T. \\
\hline 71.74 & .075 & Granitite... & Riesengebirge, Silesia .......... & N. J., 1898, XII, 232.-W.T. \\
\hline 71.19 & .073 & Quartz-porphyrite. & Calaverus County, Cal. & Bull. 168,203.-W.T. \\
\hline 70.36 & .082 & Granodiorite ..... & Enterprise, Butte County, Cul. & Bull.168, 190.-W.T. \\
\hline 69.93 & .109 & Granite-porphyry & Crazy Mountains, Nont ... & Bull. $768,120 .-$ W.T. \\
\hline 69.66 & .084 & Dacite.......... & Lassen Peak, Cal ......... & Fortieth Par., I, 652 , \\
\hline 69.56 & .076 & $\ldots \ldots$ do $\ldots \ldots \ldots$. & Colombia, South Americk ... & J.G., I, I71.-W.T. \\
\hline 69.51 & .083 & ..... do ... & Lassen Peak, Cal ...... & Bull. 168, 179.-W.T. \\
\hline 69.00 & .126 & Quartz-lindoite. & W. Aker, Norway ........ & E. K., I,198,-W.T. \\
\hline 68.72 & $.084(68.8)$ & Dacite......... & Lassen Peuk, Cul ....... & Bull. 168, 180.-W.T. \\
\hline 68.65 & .084 & Quartz-monzonite & Sierra County, Cal........... & Bull.168, 192.-W.T. \\
\hline 68.32 & .086 & Ducite....... & Lassen Peak, Cal . ..... & Bull.168, 179.-W.T. \\
\hline 68.10 & .088 & .....do.......... & Shasta County, Cal ....... & Bull, $168,180 .-\mathrm{W} . \mathrm{T}$ \\
\hline 67.89 & .088 & Andesite. & Buntingville, Cal. & Bull.168, 181.-W.T. \\
\hline 67.55 & $.087(67.2)$ & Pitchstone....... & Butte district, Mont............... & Bull. 168, 119.-W.T. \\
\hline 67.49 & $.085(67.6)$ & Dacite ................ & $\begin{array}{l}\text { Sepulchre Mountain, Yellowstone } \\
\text { Purk. }\end{array}$ & Buill. 168, 91.-W.T. \\
\hline 67.49 & .112 & Mica-dacite & Silver Gliff, Colo............. & Bull. 168, 149.-W. T. \\
\hline 67.42 & .116 & Augite-soda-granite & Kekequabic Lake, Minn .. & A. G., XI, 385.-W. T. \\
\hline 67.01 & .120 & Alaskite-porphyry. & Alasku...$\ldots \ldots \ldots \ldots \ldots$ & Bull, 168, 228.-W. T. \\
\hline 66.46 & .104 & Dacite.......... & Rosita, Colo ............. & Bull. $168,149,-$ W. T. \\
\hline 66.30 & .098 & Dacite-porphyry.. & Shast County, Cal ......... & Bull. 168, 177.-W.T. \\
\hline 66.28 & $.094(66.5)$ & Porphyrite ...... & Crazy Mountains, Nont .... & Bull. 168, 120.-W.T. \\
\hline 65.87 & .099 & Feldspar-porphyry ... & Castle Mountain district, Mont,..... & Bull. 168, 120.-W. T. \\
\hline 65.78 & .121 & Mica-andesite ........ & San Mateo Monutain, N. Mex....... & Bull. 168, 170.-W. T. \\
\hline 65.64 & .108 & Andesite-porphyry .. & Gray Peak, Yellowstone Park. & Bull. 168, 107.-W. T. \\
\hline 64.98 & .109 & Oligoclasite ......... & Preston, Norway ........... & B. M. A., 1898 , VII, 47.-W. T \\
\hline 62.58 & .109 & Syenite-porphyry. & Yogo Peak, Mont...... & Bull. 168, 127,-W. T. \\
\hline
\end{tabular}

Subrang 5. Mariposose.

(I. $4,2,5$.)

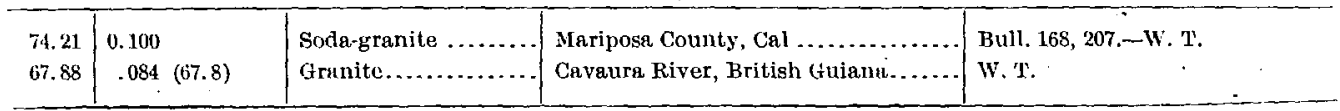


Classified list of analyses used in constructing diagrams-Continued.

- Rang 3. Coloradase.

Subrangis 3. Amatose.

(I. 4, 3, 3.)

\begin{tabular}{|c|c|c|c|c|}
\hline $\begin{array}{c}\text { Silica } \\
\text { con- } \\
\text { tent. }\end{array}$ & $\begin{array}{l}\text { Alkali-silica } \\
\text { ratio. }\end{array}$ & Author's name. & Locality. & Reference. \\
\hline 68.97 & 0.075 & Granite.. & Lower Elsass, Vosges. . & Rosenbusch, EI., 78. \\
\hline 65.53 & .085 & Trachyte..... & Monte Amiate, Italy ........... & Rosenbusch, El., 269:-W. 'T'. \\
\hline 65. 36 & .085 & Quartz-porphyrite. & Mount Carbon, Colo... & Bull. 168, 160.-W. 'T. \\
\hline 65.13 & .082 & Andesite-perlite. & Eureka, Nev.................. & U. S. G. S., Mon. XX, 264.-W. 'T. \\
\hline 63.17 & .088 & Dacite.......... & Pergamon, Asia Minor ............... & A. J. S., III, 1897, 45.-W. T. \\
\hline 61.93 & .085 & Andesite ...... & Kara Tash, Asia Minor............... & A. J. S., III, 1897, 45.-W. T. \\
\hline
\end{tabular}

SUBRANG 4. YELLOWSTONOSE.

$$
\text { (I. } 4,3,4 \text {.) }
$$

\begin{tabular}{|c|c|c|c|c|}
\hline 68.41 & 0.084 & Dacite. & Colombia, South America ............. & J. G., I, 171.-W. T. \\
\hline 68.10 & .065 & Diorite ......... & 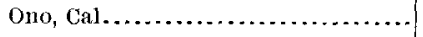 & Bull. 168, 177.-W. T. \\
\hline 68.12 & .078 & Andesite ............. & Shasta County, Cal ................ & Bull. 168, 183.-W. T. \\
\hline 67.30 & .075 & Dacite ........... & Colombis, South America. & J. G., I, 17l.-W. T. \\
\hline 66.94 & .070 & Andesite ............. & Sierra County, Cal........ & Bull. 168, 193.-W. T. \\
\hline 66.65 & .082 & $\begin{array}{l}\text { Quartz-diorite- } \\
\text { porphyry. }\end{array}$ & (..... do & Bull. 168, 192.-W. T. \\
\hline 66.65 & .074 & Granodiorite ......... & Nevada City, Cal... & Bull. 168, 194.-W. T. \\
\hline 65.71 & .065 & Bunatite....... & Banat, Hungary......... & Risenbuseh, E1., 140. \\
\hline 65.66 & .072 & Dacite .................. & $\begin{array}{l}\text { Sepulchre Mountain, Yellowstone } \\
\text { Park. }\end{array}$ & Bull. 168, 91.-W.T. \\
\hline 65.63 & .080 & Andesite............. & Fan Crcek, Yellowstone Park.. & Bull. 168, 107.-W. T. \\
\hline 65.60 & $.086(65.7)$ & Quartz-mica-diorite .. & Electric Peak, Yellowstone Park. & Bull. 168, 88.-W. T. \\
\hline 64.85 & .084 & .....do. & $\ldots$ do $\ldots \ldots \ldots \ldots \ldots \ldots$. & Do. \\
\hline 64.61 & .088 & Andesite .... & Crescent Hill, Yellowstone Park & Bull. 168, 108.-W.T. \\
\hline 64.52 & .080 & ....do... & Mount Shasta, Cal......... & Bull. 168, 176.-W. T. \\
\hline 64.48 & $.076(64.7)$ & ....do... & .... do $\ldots \ldots \ldots$. & Do. \\
\hline 64.27 & .081 & ....do. & $\begin{array}{l}\text { Sepulchre Mountain, Yellowstone } \\
\text { Park. }\end{array}$ & Bull. 168, 91.-W. T. \\
\hline 64.24 & $.078(64,1)$ & Dacite-porphyry...... & Shasta County, Cal...... & Bull. 168, 177.-W. T. \\
\hline 63. 81 & .079 & Dacite ................ & Mill Creek, Shasta County, Cal.. & Bull. 168, 180.-W. T. \\
\hline 63.42 & .098 & $\begin{array}{l}\text { Quartz-diorite- } \\
\text { porphyry. }\end{array}$ & Grandall Basin, Wyo............ & Bull. 168, 94.-W. T. \\
\hline 63.30 & .087 & Andesite ....... & Mount Rose, Washoe, Nev .... & Fortieth Par. I, 604. . \\
\hline 62.91 & .094 & Granitite ............ & Dorseys Run, Md............ & Bull. 168, 47.-W. T. \\
\hline 62.85 & .085 & (?) Porphyrite-diorite & Mount Marcellina, Colo. . & Bull. 168, 160.-W. T. \\
\hline 61.42 & .078 & Yorphyrite ... & Storm Ridge, Colo... & Do. \\
\hline
\end{tabular}

Subrang 5. Amadorose.

$$
\text { (I. } 4,3,5 \text {.) }
$$

\begin{tabular}{|c|c|c|c|c|}
\hline 69.66 & 0.074 & Quartz-diorite-aplite. & Amador County, Cal. & Bull. 168, 201.-W. T. \\
\hline 69.34 & .066 & Granite............... & Sagunaga Lake, Minn............ & $\begin{array}{l}\text { 21st Ann. Rept. Geol. Surv. Mints., } \\
\text { 43.-W. T. }\end{array}$ \\
\hline
\end{tabular}

RANG 4.

SUBRANG 3. -

(I. $4,4,3$.

\begin{tabular}{l|l|l|l|l|}
\hline 65.81 & 0.047 & Granite............. & Hochwald, Vosges................. & $\begin{array}{c}\text { Rosenbusch, Steiger Sch i e fer } \\
\text { (Strussburg, } 1877), 167 .\end{array}$ \\
\hline
\end{tabular}


Classified list of analyses used in constructing diagrams-Continued.

\section{Order 5. Canadare.}

RaNg 1. Nordiarkase.

SUBRANG 3. PHLEgROSE.

(I. $5,1,3$. )

\begin{tabular}{|c|c|c|c|c|}
\hline $\begin{array}{l}\text { Silica } \\
\text { cont- } \\
\text { tent. }\end{array}$ & $\begin{array}{l}\text { Alkali-silica } \\
\text { ratio. }\end{array}$ & Author's name. & Locality. & Reference. \\
\hline 665.13 & 0.130 & Quartz-syenite. & Fjelebua, Norway. . & Z. K., XVI, 46. -W. T. \\
\hline 66.03 & $.132(6 \overline{\operatorname{s}} .9)$ & Truchyte.... & Game Ridge, Custer County, Colo & Bull. 168, 147.-W. T. \\
\hline 65.43 & .131 & Syenite........ & 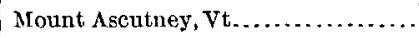 & Bull. 168, 24.-W. T. \\
\hline 64.88 & .128 & Syenite-porphyry & $\ldots$. do $\ldots \ldots \ldots \ldots$ & Bull. I68, 25.-W. T. \\
\hline 64.04 & .160 & Nordmarkite..... & Tonsenas, Norway ...... & Z. K., XVI, 54.-W. T. \\
\hline 63.71 & .159 & Pulaskite...... & Salem Neck, Mass.... & J. G., VI, 806,-W. T. \\
\hline 62.30 & .142 & Bostonite.... & Gran, Norway ........ & E. K., III, 204.-W. T. \\
\hline 61.92 & .151 & Pulaskite.... & Lokobe, Madagascar... & M. M., 204. \\
\hline 61.88 & .176 & Trachyte..... & Marecocco, Italy .............. & A. J. S., VIII, 1899, 289.-W. T. \\
\hline 61.62 & $.170(61.2)$ & .....do . . & Monte Rotaro, Italy ....... & Do. \\
\hline 61.45 & $.143(61.3)$ & .....do ........ & Maros, Celebes............ & G. C., 16. \\
\hline 61.05 & .166 & Sölvsbergite... & Coney Island, Mass....... & J. G., VII, 118.-W. T. \\
\hline 60.33 & .193 & Trachyte ..... & Monte Nuovo, Italy ........... & A. J. S., VIII, 1899, 287.-W. T. \\
\hline 60.13 & .165 & Nephelite-syenite... & Fourche Mountain, Ark....... & J. G., IX, 610.-W. T. \\
\hline 60.02 & .147 & Syenite-keratophyre. & Harz Mountains....... & R. T., 1884, XXII. \\
\hline 59.79 & .186 & Trachyte,......... & Monte Nuovo, Italy ......... & A. J. S., VIII, $1899,287 .-$ W. T. \\
\hline 59.70 & .164 & Sephelite-syenite .... & Fourche Mrountain, Ark.. & I. R. A., 88.-W. T. \\
\hline 58.75 & .176 & Umptekite ........... & Cabo Frio, Brazil . .............. & T. M. P. M., XX, 248.-W. T. \\
\hline 57.18 & .171 & Trachyte.... & Highwood Mountains, Mont.. & Bull. 168, 131.-W. T. \\
\hline
\end{tabular}

Subrang 4. Norditakose.

(I. $5,1,4$.

\begin{tabular}{|c|c|c|c|c|}
\hline 66.50 & 0.162 & Lusti wurite. & Kvella, Norway & E. K., III, 216. -W. T. \\
\hline 66.22 & $.149(66.4)$ & $\begin{array}{l}\text { Qnartz - syenite - por- } \\
\text { phyry. }\end{array}$ & Benrpaw Mountains, Mont. & Bull. 168, 135.-W. T. \\
\hline 66.06 & .151 & Liparite ............ & Hohenburg, near Bonn, Prussia.... & R. T., 1884, XLVIII. \\
\hline 65.51 & .143 & Phonolitic undesite & San Mateo Mountains, N. Mex. & Bull, 168, 170.-W. T. \\
\hline 65.43 & $.140(65.1)$ & Nordmarkite .... & Shefford Mountain, Quebee.. & J. G., XI, 271. \\
\hline 64.92 & .146 & Quartz-sölvsbergite & Gran, Norway ....... & F. K., I, 78.-W. T. \\
\hline 64.54 & .157 & Pulaskite........ & Lovasbucht, Norway.... & E. K., III, 198.-W. T. \\
\hline 64.33 & $.151 \cdot$ & Aemite-trachyte. & Crazy Mountains, Mont........... & Bull. 168, 123.-W. T. \\
\hline 63.76 & $.161(68.4)$ & Syenite....... & Ahvenvaara, Finland .... & B. C. G. F., 11, 34.-W. T. \\
\hline 63.20 & .164 & Nordmarkite.... & Tonsenas, Norway......... & Z. K., XVI, 54.-W.T. \\
\hline 63.09 & $.164(63.6)$ & Pulaskite... & Salem Neck, Mass ........ & I. G., VI, 806. -W. T. \\
\hline 62.17 & .156 & Acmite-trachyte. & Grazy Mountains, Mont ........ & Bull. 168, 123.--W. T, \\
\hline 61.67 & .169 & Keratophyre. & Harz Mountuins...... & R. T., 1884, XXII. \\
\hline 61.54 & .180 & Phomolite..... & Zittalı, Saxony ........... & R. T., 1861, 23. \\
\hline 61.08 & .185 & ..... do $\ldots .$. . & Black Hills, S. Dak............ & A. J, S., XLVII, 1894, 344.-W.T. \\
\hline 60.39 & .184 & Litchfieldite.. & Litchfield, Me................. & Bull. 168, 21.-W. T. \\
\hline 60.11 . & $.161(59.9)$ & Bostonite.. & Hedrum, Norway ........ & E. K., 1II, 204.-W. T. \\
\hline 60.05 & $.157(50.3)$ & Tinguaite ........... & Gales Point, Mass ............. & J. G., VII, 481,-W. T. \\
\hline 59.62 & .173 & Nepholite-syenite.... & Saline County, Ark.......... & I. R. A., 135.-W. T. \\
\hline 59.81. & .174 & Foyaite..... & Grent Haste Island, Mass.......... & J. G., VII, 481.-W. T. \\
\hline
\end{tabular}


Classified list of analyses used in constructing diagrams-Continued.

Subrang 5. Tuolimnose.

$(\mathbf{I}, 5, \mathbf{1}, \mathbf{5}$.)

\begin{tabular}{|c|c|c|c|c|}
\hline $\begin{array}{l}\text { Silich } \\
\text { cont- } \\
\text { tent. }\end{array}$ & $\begin{array}{l}\text { Alkali-silica } \\
\text { ratio. }\end{array}$ & Author's name. & Locality. & Reference. \\
\hline 67.53 & 0.165 & Albitite...... & Trolumne County, Cal ............... & Bull. 168, 204.-W. T. \\
\hline 66.09 & .162 & $\ldots . . . d o \ldots$ & Koswinsky, Ural Mountaiıs......... & O. N., 169. \\
\hline
\end{tabular}

Rang 2. Pulaskase.

SURRANG 2. VULSINOSE.

(I. $5,2,2$.

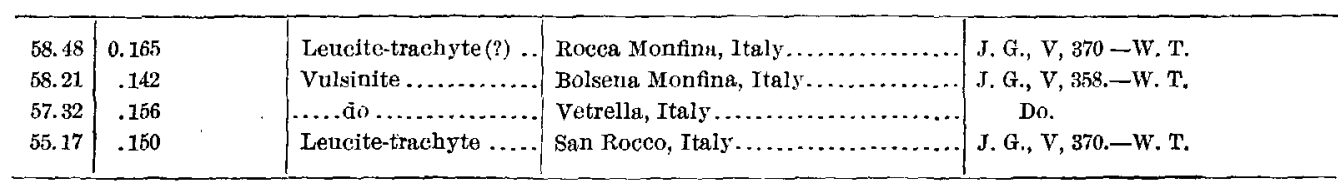

Subrang 3. Pulaskosz.

(I. $5,2,3$.

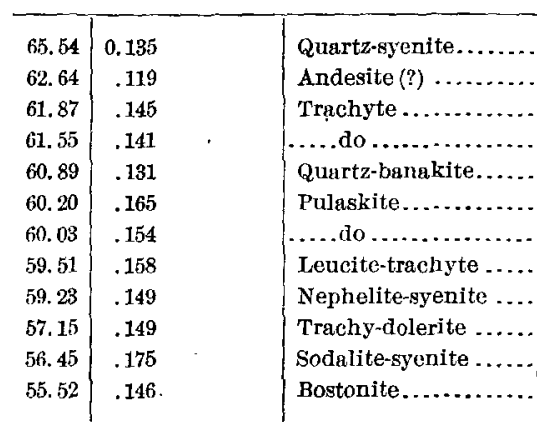

Highwood Mountains, Mont......... $\mid$ w. T

Pikes Peak distriet, Colo ............. Bull. 168, 145.,-W. T.

Ischiu, Italy ...................... R. T., 1873, XXXVIIf.

.....do ............................ R. T., 1873, XXXVI.

Ishawook Canyon, wyo ............. Bull. 168, 102.-W.T.

Fourche Mountain, Ark............. J. G., IX, 609.--W.T.

.... do .......................... I. R. A., 88. -W. T.

Viterbo, Italy .................... J. G., V, 370.-W. T.

Fourche Mountain, Ark............ I. R.A., 88.-W.T.

Maros, Celebes ..................... G. C., 14.

Square Butte, Mont................ Bull, 168, 134.-W. $T$.

Gentungen, Celebes................ G. C., 20.

Subrang 4. Laurvikose.

( I. $5,2,4$.

\begin{tabular}{|c|c|c|c|c|}
\hline 65.41 & 0.139 & Trachyte.......... & Game Ridge, Custer County, Colo... & Bull. 168, 147.-W.T. \\
\hline 64.80 & .125 & Domite ........... & Puy de Dome, France... & R. T., 1869, CXVIII. \\
\hline 64.63 & .132 & Quartz-syenite. & Fourche Mountain, . Ark. & I, R. A., 96. -W. T. \\
\hline 63.49 & .183 & Andesite... & Custer County, Colo..... & Bull. 168, 148.-W.T. \\
\hline 61.47 & .120 & ..... do... & Pantellaria, Italy.. & Z. K., VIII, 155. \\
\hline 61.43 & .133 & .....do ..... & .....do............ & Z. K., VIII, 164. \\
\hline 61.05 & .147 & Trachyte. & Ischia, Italy ... & R. T., 1873, XXXVIII. \\
\hline 60.98 & $.142(59.9)$ & Augite-porphyrite... & Henry Mountains, Utah. & Bull. J68, 167.-W.T. \\
\hline 60.72 & .142 & Rhombenporphyry... & Nötterö, Norway. & Z. K., XVI, 35.-W.T. \\
\hline 60.45 & .168 & Nordmarkite ........ & Aneröd, Norway ......... & Z. K., XVI, 54.-W.T. \\
\hline 60.24 & .170 & Augite-andesite & Pantellaria. & Z. K., VIII, 155. \\
\hline 59.96 & .165 & Pulaskite....... & Shefford Mountaiu, Quebec. & J.G., XI, 271. \\
\hline 59.38 & .125 & Tönsbergite. & Tönsberg, Norway..... & E. K., III, 375.-W. T. \\
\hline 58.94 & .109 & Andesite...... & Silver Cliff, Colo........ & Bıt1. 168, 148.-W.'T. \\
\hline 58.88 & .141 & Laurvikite & Laurvik, Norway.. & Z. K., XVI, 30.-W. T. \\
\hline 58.82 & .155 & Rhombenporphyry & Tönsberg, Nórway .. & Z. K., XVI, $35 .-W . T$. \\
\hline 57.59 & .139 & ....do... & Nötterö, Norway, ............. & Do. \\
\hline 57.44 & .157 & Pulaskite... & Mount Johnson, Quebec ...... & J. G., XI, 271. \\
\hline
\end{tabular}


Classified list of analyses used in constructing diagrams-Continued.

Subrang 4. LaURyik Rose-Continued.

\begin{tabular}{|c|c|c|c|c|}
\hline $\begin{array}{l}\text { Silica } \\
\text { con- } \\
\text { tent. }\end{array}$ & $\begin{array}{l}\text { Alkalí-silica } \\
\text { ratio. }\end{array}$ & Author's name. & Locality. & Reference. \\
\hline $\begin{array}{l}57.33 \\
57.12 \\
56.85 \\
56.25 \\
56.19 \\
54.00\end{array}$ & $\begin{array}{l}.153(5 \pi .1) \\
.138 \\
.144 \\
.155(55.9) \\
.156 \\
.162\end{array}$ & $\begin{array}{l}\text { Rhombenporphyry ... } \\
\text { Laurvikite ............ } \\
\text { Micromongonite.................... } \\
\text { Tephritic trachyte.... } \\
\text { Rhombenporphyry ... }\end{array}$ & 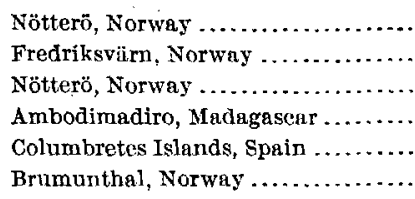 & $\begin{array}{l}\text { Z. K., XVI, 35.-W. T. } \\
\text { Z. K., XVI, 30.-W. T. } \\
\quad \text { Do. } \\
\text { M. M., 204. } \\
\text { T. M. P. M., XVI, 314. -W. T. } \\
\text { Z. K., XVI, 28.-W. T. }\end{array}$ \\
\hline \multicolumn{3}{|c|}{ • } & $\begin{array}{l}\text { SUBRANG } 5 . \overline{ } \\
\text { (I. } 5,2,5 .)\end{array}$ & \\
\hline $\begin{array}{l}62.30 \\
60.13\end{array}$ & $\begin{array}{r}0.135 \\
.164\end{array}$ & $\begin{array}{l}\text { Diorite } \ldots \ldots \ldots \ldots \ldots \\
\text { Keratophyre.......... }\end{array}$ & $\begin{array}{l}\text { Jablanica, Herzegovina ............. } \\
\text { New Haven, Conn } \ldots \ldots \ldots \ldots \ldots \ldots \ldots\end{array}$ & $\begin{array}{l}\text { W. T. } \\
\text { A. J. S., III, 1897, 291.-W. T. }\end{array}$ \\
\hline
\end{tabular}

RANG 3,

SUBRANG 2. -

(I. $5,3,2$ )

\begin{tabular}{|c|c|c|c|c|}
\hline 69.33 & $0.135(59.5)$ & Augite-syenite.. & Mazaruni district, British Guiana.... & W. T. \\
\hline
\end{tabular}

SUBRAXIG 3. -

(T. $5,3,3$.

\begin{tabular}{|c|c|c|c|c|}
\hline 59.26 & 0.139 & Andesite ..... & Table Mountain, Colo ............... & Bull. 168, 141.-W. T. \\
\hline \multicolumn{5}{|c|}{$\begin{array}{l}\text { SUBRANG } 4 .- \\
(1.5,3,4 .)\end{array}$} \\
\hline 58.28 & 0.112 & Diabase-porphyrite... & Crazy Mountains, Mont ........ & Bull. $168,121 .-W . T$. \\
\hline
\end{tabular}

Rang 4. Labradorase.

SUBRANG 3. LABRADOROSF.

(T. $5,4,3$.)

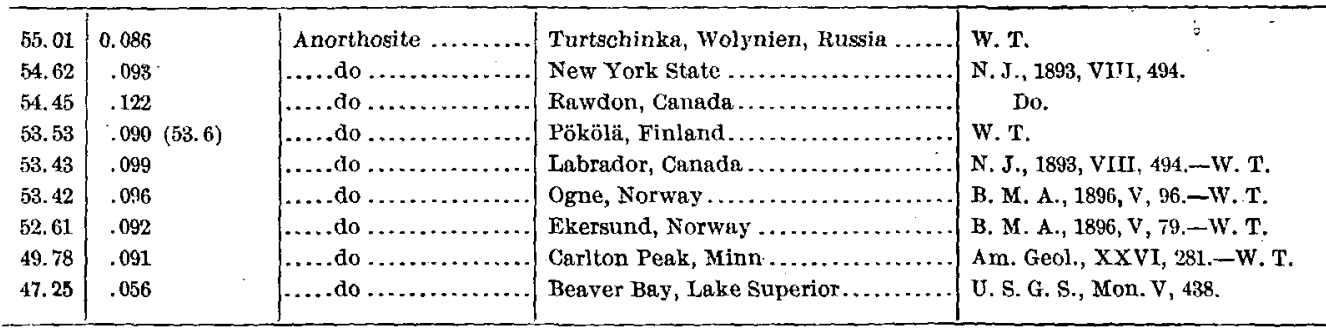

Rang 5. Canadase.

(I. $5,5$.

\begin{tabular}{|c|c|c|c|c|}
\hline 47.32 & $0.048(47.4)$ & Anorthosite ......... & South Sherbrooke, Ontario........... & Am. Geol., XXIV, 280.-W. T. \\
\hline 46.24 & .044 & ... do . . . . . . & Seine River, Canada........ & J. G., IV, 909.-W. T. \\
\hline 45.78 & .036 & .....do. & Monhegan Isle, Me.. & Am. Geol., XXVI, 340.-W. T. \\
\hline
\end{tabular}

14474-No. $18-03-3$ 
Order 6. Russare.

Rang 1. Miaskase.

Subraxg 3. BeEMERose.

$$
\text { (I. } 6,1,3 .)
$$

\begin{tabular}{|c|c|c|c|c|}
\hline $\begin{array}{c}\text { Silica } \\
\text { con- } \\
\text { tent. }\end{array}$ & $\begin{array}{l}\text { Alkali-silica } \\
\text { ratio. }\end{array}$ & Author's name. & Locality. & Reference. \\
\hline 60.42 & 0.184 & Nephelite-syenite & Moita, Foya, Portugal ..... & T. M. P. M., XV1, 225.-IV. I'. \\
\hline $59.20^{\circ}$ & $.189(59.1)$ & Mieroditroite.... & Nosy Komba, Madagasear..... & M. M., 169. \\
\hline 58.89 & .180 & $\begin{array}{l}\text { Leucite - tingua ite- } \\
\text { vitrophyre. }\end{array}$ & Picota, Portugal...... & T. M. P. M., XVI, 252. -W.T. \\
\hline 58.72 & $.199(58.8-.200)$ & Ditroite.............. & Nosy Komba, Madagascar... & M. Mf., 204. \\
\hline 58.00 & .199 & Phonolite ........... & Marus, Celebes ..... & G. C., 18 . \\
\hline 55.87 & $.201(55.3)$ & Leucite-phonolite.... & Lake Bracciano, Italy .... & J.G., V., $370 .-$ W. T. \\
\hline 55.38 & .207 & Foyaite.. & East Cape, Sibcria... & A.J.S., XIII, 1902, 176. -W.T. \\
\hline 53.56 & $.229(53.1)$ & Nephelite-syenite.... & Becmerville, N. J.................. & Bull. $168,39 .-$ W. T. \\
\hline 53.09 & .226 & Foyaite......... & Magnet Cove, Ark.................. & J.G., IX,611. -W.T. \\
\hline
\end{tabular}

Sobrang 4. Mrashose.

(I. $6,1,4$.)

\begin{tabular}{|c|c|c|c|c|}
\hline 60.02 & 0.202 & Phonolite & Cripple Creek, Colo.. & Bu11.168, 143. -W.T. \\
\hline 59.38 & .189 & Trachytic phonolite. & .... do .... & Bull, $168,144,-$ W. T. \\
\hline 59.00 & .196 & Phonolite $\therefore$ & $\ldots \ldots$ do $\ldots . . . . .$. & Bull.168, 143.-W.T. \\
\hline 58.98 & .219 & ..... do... & .... do ........... & Do. \\
\hline 58.78 & .213 & ....do do..... & $\ldots \ldots$ do $\ldots \ldots \ldots$ & Do: \\
\hline 58.77 & $.211(59.1)$ & Foyaite... & Salem Neck, Mass ....... & J. G., VII, 481. - IV.T. \\
\hline 58.74 & .205 & Nephelite-syenite. & Salina County, Ark..... & I. R.A., 139. -W.T. \\
\hline 58.70 & .190 & Acmite-trachyte. & Grazy Mountains, Mont. & Bull. $168,123 .-W . T$. \\
\hline 58.64 & $.195(, 196)$ & Fhonolite ........ & Cripple Creek, Colo....... & Bull. J68, 143. -W.T. \\
\hline 58.62 & $.191(58.5-18 x)$ & .... do ........... & Nosy Komba, Madagascar. & M. M., 169. \\
\hline 58.61 & $.194(58.3)$ & Foyaite..... & Heum, Lougenthal, Norway & E. K., III, 377. -W.T. \\
\hline 58.61 & $.213(58.5)$ & ..... do ........ & Nosy Komba, Mudagascar.. & N. $\mathrm{MI}, 169$. \\
\hline 58.51 & $.216(58.2)$ & Phonolite ......... & Velay, France............. & R.'T., 1879, LVIII. \\
\hline 58.25 & $.197(57.7)$ & Microfoyaite & Nosy Komba, Madagasear. & M. M., 169. \\
\hline 58.20 & .190 & Ditroite....... & ... do do................. & Do. \\
\hline 58.10 & $.191(57.9)$ & ....do..... & {$[\ldots$. do $\ldots . . . . .$.} & M. М., 204. \\
\hline 57.86 & $.214(57.8)$ & Phonolite ......... & Black Hills, S. Dak........ & Bull. 168, 84.-W. T. \\
\hline 56.75 & .228 & Tinguaite .. & Pickards Point, Mass.. & J. G., VII, 481.--W. T. \\
\hline 56.67 & $.226(56.3)$ & Nephelite-syenite. & Picota, Portugal. ....... & T. M. P. M., XV1, 228.-W. T. \\
\hline 56.43 & $.195(56.3)$ & Phonolite.. & 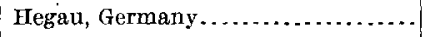 & R. T., 1884, LIV. \\
\hline 56.40 & .201 & Nophelite-syenite ... & Poutelitschorr, Kola, Finland.. & Rosenbusch, El., 126.-W. T. \\
\hline $56 \dot{3} 30$ & .235 & Ditroite .. & Ditro, Siebenbürgen, Hungary. & Rosenbuseh, E1,, 126. \\
\hline 56.26 & $.199(56.8)$ & Miascite.... & Mount Sobatehia, Siberia & W. T. \\
\hline 55.95 & .256 & Phonolite... & Borczen, Bilin ..... & R. T., 1869, XCVI. \\
\hline 55.93 & $.203(55.6)$ & Trachytic phonolite.. & Columbretes Islands.... & T. M. P. M., XVI, 314.-W. T. \\
\hline 65.92 & $.198(55.8)$ & Phowolite ............ & Hegan, Germany......... & R. T., 1884, LII. \\
\hline 65.21 & .226 & .....do .... & $\ldots \ldots$ do $\ldots . . . . . . .$. & R. T., 1879, LVIII. \\
\hline 55.01 & .211 & ..... do ..... & $\ldots \ldots$ do $\ldots . . . . . . . .$. & R. T., 1884, LII. \\
\hline 54.92 & .243 & Nophelite-aplite..... & Cabo Frio Island, Brazil. & T. M. P. M., XX, 288.-W. T. \\
\hline 54.22 & .224 & Phonvlite ............ & Southboro, Mass.......... & Bull. 168, 33.-W. T. \\
\hline 54.20 & .237 & Nephelite-syenite.. & Picota, Portugal... & T. M. P. M., XVI, 218.-W. T. \\
\hline 53. 95 & .246 & Phonolite ............. & 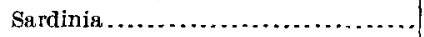 & R. T., 1879, LVIII. \\
\hline 53.65 & .231 & ....do....... & Msid Gharian, Tripoli.. & R. T., 1884, LIV. \\
\hline 52.73 & .202 & Heronite ..... & Heron Buy, Canada ...... & J. G., VIT, $435 .-W . T$. \\
\hline
\end{tabular}


Classified list of analyses used in constructing diagrams-Continued.

Subrayg 5. MariUPolose.

(I. 6, 1, 5.)

\begin{tabular}{|c|c|c|c|c|}
\hline $\begin{array}{l}\text { Silica } \\
\text { con- } \\
\text { tent. }\end{array}$ & $\begin{array}{l}\text { Alkali-silica } \\
\text { ratio. }\end{array}$ & Author's name. & Locality. & Reference. \\
\hline 60.29 & $0.197(60.5)$ & Mariupolite ..... & Nikolajew, Russia............. & T. M. P. M., XXI. 244.-W. T. \\
\hline$\cdot$ & & & $\begin{array}{l}\text { RaNG 2. Viñzzenase. } \\
\text { SUbraNG } 3 .\end{array}$ & , \\
\hline 53.76 & $0.207(53.6)$ & Nephelite-tinguaite.. & Magnet Cove, Ark.......... & I. R. A., 260.-W. T. \\
\hline \multicolumn{5}{|c|}{$\begin{array}{c}\text { SUPRANG 4. VIEqZZENOSE. } \\
\text { (I. } 6,2,4 .)\end{array}$} \\
\hline 56.04 & 0.201 & $\begin{array}{l}\text { Nephlite-rhomben - } \\
\text { porphyry. }\end{array}$ & Vasvik tunnel, Norway & Z. K., XVI, 38.-W. T. \\
\hline 55.40 & .184 & Andesite ............. & Palma, Sicily ......... & R. T., 1879, LXVI. \\
\hline 54.68 & .176 & Nephelite-syenite .... & 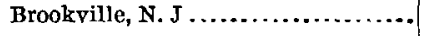 & Bull. 168, 39.-W. T. \\
\hline 54.61 & .197 & ...... dò ................... & Caldas de Monchique, Portugal...... & N. J.; B. B., III, 271.-W. T. \\
\hline 54.46 & .186 & Tinguaite ............. & Umptek, Kola, Finland .............. & Rosenbusch, El., 215.-W. T. \\
\hline
\end{tabular}

Order 7. Tasmanare.

Rang 1. Laughase.

SUBRANG 4. ĹaUgenOSE.

(I. $7,1,4$.

\begin{tabular}{l|l|l|l|l|}
\hline 65.50 & 0.248 & Foyaite.............. & Brathagen, Norway ............... E. K., III, 176. -W. T. \\
\hline
\end{tabular}

Class II. DOSALANE.

Order 3. Hispanate.

Rang i. Varingase.

SUBHANG 3. VARINGOSE.

(II. $3,1,3$.$) .$

\begin{tabular}{|c|c|c|c|c|}
\hline 74.35 & 0.092 & Grorudite ..... & Varingskollen, Norway .............. & Z. K., XVI, 66.-W. T. \\
\hline 70.30 & .128 & Pantellerite .......... & Pantellaria ........................ & Z. K., VIII, 173.-W. T. \\
\hline
\end{tabular}

SUBRANG $4 .-$

(II. $3,1,4$.

\begin{tabular}{|c|c|c|c|c|c|}
\hline 72.12 & 0.070 & Felsite.................. & $\begin{array}{l}\text { Cudgegong River, New South Wales, } \\
\text { Australia. }\end{array}$ & $\mathbf{w} . \mathbf{T}$. & $\therefore$ \\
\hline
\end{tabular}


Classified list of analyses used in constructing diagrams-Continued.

RANG 4.

SUBRANG 3

(II. $3,4,3$.)

\begin{tabular}{l|c|c|c|c}
\hline $\begin{array}{c}\text { Silica } \\
\text { con- } \\
\text { tent. }\end{array}$ & $\begin{array}{c}\text { Alkali-silica } \\
\text { ratio. }\end{array}$ & Author's name. & Locality. & \multicolumn{1}{c}{ Reference. } \\
\hline 66.88 & 0.020 & Quartz-diorite ......... & Little Falls, Minn ................... R. T., 1879, XXXVI. \\
\hline
\end{tabular}

Order 4. Austrare.

Rang 1. Pantellerase.

SUBRANG 3. GroRUdose.

(II. 4, 1, 3.)

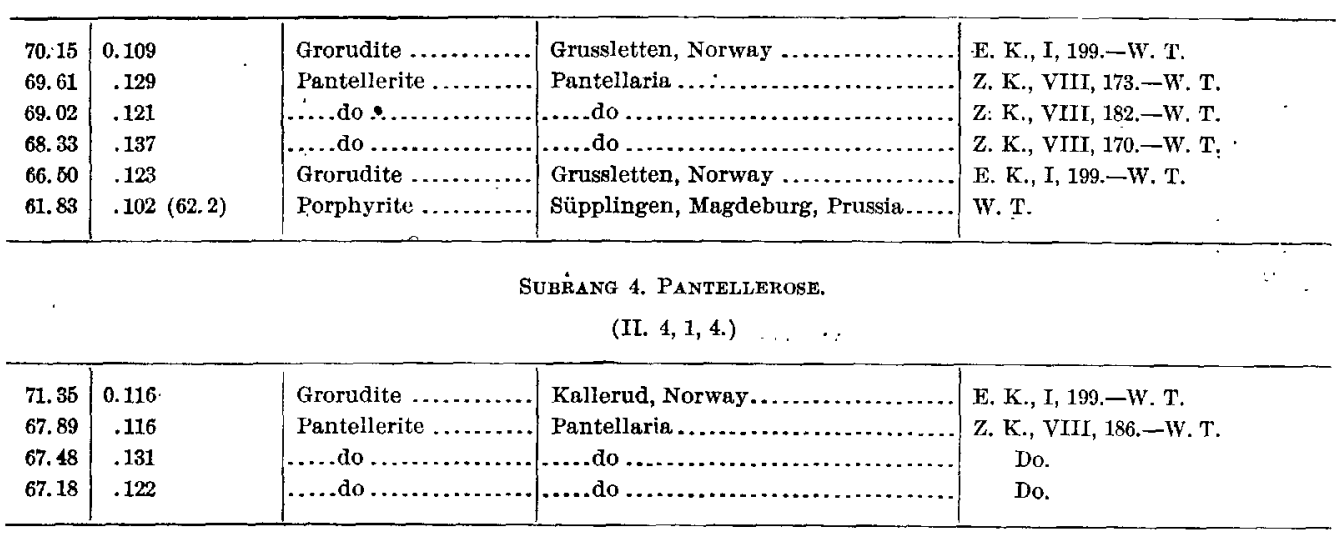

Rang 2. Dacase.

Subrang 3. Adamellose.

(II. $4,2,3$.

\begin{tabular}{|c|c|c|c|c|}
\hline 66.57 & 0.103 & Granite-porphyry .... & Kirche Wang, Silesia.. & Rosenbusch, El., 195. \\
\hline 63.97 & .105 & Quartz-mica-diorite .. & Crandall Basin, Wyo .. & Bull. 168, 94.-W. 'I'. \\
\hline 62.51 & .090 & Syenite........ & Reichenstein, Silesia . & N. J., 1890, I, 206.-W. T. \\
\hline 61.93 & .104 & Granitite .... & Laveline, Vosges ............... & Rosenbusch, El., 78 \\
\hline 59.24 & $.095(59.5)$ & Trachyte-andesite.... & Highwood Mountains, Mont ........ & Bull. 168, 131.-W. T. \\
\hline
\end{tabular}

SUbrang 4. DACOSE.

(II. 4, 2, 4.)

\begin{tabular}{|c|c|c|c|c|}
\hline 65.50 & 0.107 & Andesite ...... & $\begin{array}{l}\text { Sepulchre Mountain, Yellowstone } \\
\text { Park. }\end{array}$ & Bull. 168, 91.-W. T. \\
\hline 64.47 & .098 & Granitite... & Crazy Mountains, Mont . . . . . . . . . . . . & Bull. $168,120 .-W$. T. \\
\hline 68.50 & .101 & Dreite.... & Chiles, Colombia, South America .... & J. G., I, 171.-W. T. \\
\hline 61.56 & .102 & Andesite .. & Tower Creek, Yellowstone Park ..... & Bull. 168, 108.-W. T. \\
\hline 56.59 & .083 & Quartz-diorite. & 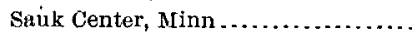 & R. T., $1879, \mathrm{XXXIV.}$ \\
\hline
\end{tabular}


ROCK ANALYSES USED IN CONSTRUCTING DIAGRAMS.

Classified list of anrlyses $u$ ised in constructing diagrams-Continued.

Rang 3. Tonalase.

Subrang' 3. HaRzose.

(II. $4,3,3$.)

\begin{tabular}{|c|c|c|c|c|}
\hline $\begin{array}{l}\text { Silica } \\
\text { con- } \\
\text { tent. }\end{array}$ & 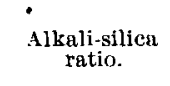 & Author's name. & Locality. & Reference. \\
\hline 64.34 & 0.081 & Granite.. & Butte, Mont .... & Bull. 168, 117.-W. 'T. \\
\hline 63.85 & .080 & Granodiorite... & Grass Valley, Cal... & Bull, $168,194 .-W . T$. \\
\hline 63.06 & $.069(63.2)$ & "Schliere" in granite. & Riesengebírge, silesia .. & N. J., 1898, XII, 236.-W. T. \\
\hline 61.73 & .088 & Syenite ............ & Hodritch, Hungary........ & R. T., 1869, LX. \\
\hline 61.64 & .078 & Quartz-monzonite.... & Red Rock Creek, Butte, Mont. . & Hull. $168,118 .-$ W. 'T. \\
\hline 60.68 & $.090(60.7)$ & Andesite ....... & Mount Pagos, Asia Minor . & A. J. S. III, 1897, 43.-W. T. \\
\hline 60.17 & .090 & Latite .......... & Tintic district, Utah........ & Buil. 168, 166. -W. T. \\
\hline $59.76^{\circ}$ & .094 & Monzonite........ & $\ldots$ do $\ldots \ldots . . .$. & - Do. \\
\hline 57.80 & .068 & $\begin{array}{l}\text { Quartz-pyroxene-dio- } \\
\text { rite. }\end{array}$ & Sonora, Tuolumne County, Cal... & Bull. 168, 204.-W. T. \\
\hline 57.69 & $.064(58 . i)$ & Mica-diorite......... & Gippsland, Victoria... & E. K., II, 37.-W. T. \\
\hline 57.26 & .076 & Quartz-mica-diorite .. & Sierra County, Cal. & Bull. 168, 192.-W. T. \\
\hline
\end{tabular}

Subrang 4. TONalose.

(II. $4,3,4$ )

\begin{tabular}{|c|c|c|c|c|}
\hline 66.91 & 0.055 & Tonalite. & Adamello Stock, Tyrol. & Rosenbusch, El, 140.-W.T. \\
\hline 65.83 & .080 & Granite. - & Granite Creek Station, Nev & Fortieth Par., I, 110. \\
\hline 65.39 & .081 & Dacite.... & Cumbal, Colombia, South America. & J, G., I, 171.-W.T. \\
\hline 65.11 & $.0 \$ 3$ & Quartz-mica-diorite.. & Electric Peak, Yellowstone Park. & Bull, 168, 88.-W. T. \\
\hline 64.81 & .073 & Porphyrite ........... & Leadville district, Colo............ & Bull. 168, 155.-W. T. \\
\hline 64.12 & $.077(.076)$ & Quartz-norite. & Tyrol . . . ............. & Rosenbusch, El., r40. \\
\hline 64.07 & .083 & Quurtz-mica-diorite & Electric Peak, Yellowstone Park..... & Bull, 168, 87.-W. T. \\
\hline 63.56 & .084 & Hornblende-dacite. & Colombia, South America...... & J. G., 1, 171.-W.T. \\
\hline 63.47 & .075 & Andesite .... & $\begin{array}{l}\text { Suppans Mountain, Tehama County, } \\
\text { Cal. }\end{array}$ & Bull. 168, 182.-W. T. \\
\hline 63.28 & .077 . & ..... do. & Mount Hood, Oreg .............. & Forticth Par., I, 604. \\
\hline 63.16 & .089 & Trachyte... & Henry Mountains, Utah. . & Bull. 168, 167.-W. T. \\
\hline 63.03 & .070 & Andesite ...... & Mount Shasta, Cal............. & Bull. 168, 176.-W. T. \\
\hline 62.71 & .084 & Diorite ..... & Gunnison County, Colo..... & Bull. 168, 159.-W. T. \\
\hline 62.65 & .095 & $\begin{array}{l}\text { Hornblende-porphy- } \\
\text { rite. }\end{array}$ & Herman Peak, Colo ............ & Bull. 168, 164.-W. T. \\
\hline 62.62 & .070 & Quartz-mica-diorite.. & Mariposa County, Cul .... & Bull. 168, 209.-W. T. \\
\hline 62.44 & .071 & Andesite ....... & Shusta County, Cal ........ & Bull, 168, 181.-W. T. \\
\hline 62,09 & .070 & $\begin{array}{l}\text { Hornblende-porphy- } \\
\text { rite. }\end{array}$ & Nevada City, Cal .............. & Bull. 168, 194.-W. 'T'. \\
\hline 61.58 & $.074(61.8)$ & Andesite & Mount Shusta, Cal..... & Bull. 168, 176,-W. T. \\
\hline 61.40 & .048 & Syènite.. & Riesengebirge, Silesia......... & N. J., 1898, XII, $234 .-W$. T.. \\
\hline 61.22 & $.089(61.4)$ & Mich-diorite.... & Electric Peak, Yellowstone Park..... & Bull. 168, 87.-W. T. \\
\hline 61.17 & .077 & Andesite...... & Shasta County, Ca1 ............ & Bull. $168,183 .-\mathrm{W}, \mathrm{T}$. \\
\hline 61.16 & .093 & Andesite-porphyry ... & Crandall Basin, wyo ......... & Bull. 168, 94.-W. T. \\
\hline 61.09 & .070 & Andesite ... & Colombia, South America.... & J. G., I, 171.-W. T. \\
\hline 61.04 & .090 & .....do. & .....do $\ldots . . . . . . . . . . . . . .$. & Do. \\
\hline 60.93 & .073 & ....do. & Tuscan Buttes, Cal ............. & Bull. 168, 181.-W. T. \\
\hline 60.30 & $.088(60.4)$ & .....do.. & $\begin{array}{l}\text { Sepulchre Mrountain, Yellowstone } \\
\text { Park. }\end{array}$ & Bull. 168, 90.-W. T. \\
\hline 60.20 & .068 & ....., do & Pìlot Peak, Plumas County, Cal.. & Bull. 168, 188.-W. T. \\
\hline 60.05 & .079 & ..... do. & Colombia, South America....... & J. G., I, 171.-W. T. \\
\hline 60.04 & $.078(60.3)$ & $\ldots$ do & Shasta County, Cal.... & Bull, 168, 181,-W. T. \\
\hline 60.02 & $.074(59.8)$ & ....do. & Sierra County, Cal.... & Bull. 168, 193.-W. T. \\
\hline
\end{tabular}


Classified list of analyses used in constructing diagramy-Continued.

Subrang 4, Tonalosk-Continued.

\begin{tabular}{|c|c|c|c|c|}
\hline $\begin{array}{l}\text { Silica } \\
\text { con- } \\
\text { tent. }\end{array}$ & $\begin{array}{l}\text { Alkali-silica } \\
\text { ratio. }\end{array}$ & Author's name. & Locality. & Reference. \\
\hline 59.97 & .076 & Diorite & Vildarthal, Tyrol... & Rosenbusch, El.; 140. \\
\hline 59.84 & .080 & Andesite............. & Near Iassen Peak, Cal ..... & Bull. 168, 183.-W. T. \\
\hline 69.77 & .088 & $\begin{array}{l}\text { A vernge of } 680 \text { igne- } \\
\text { ous rocks. }\end{array}$ & America............... & Bull. 148, 12.-W. T. \\
\hline 59.48 & .082 & Andesite.. & Red Bluff, Mont.. & Bull. 168, 114.-W. T'. \\
\hline 59.34 & .075 & ..... do .......... & Sierra County, Cal.............. & Bull, 168, 193.-W. T. \\
\hline 59.19 & .094 & Diorite......... & San Miguel Mountains, Colo ......... & Bull. 168, 164.-W. T; \\
\hline 58.67 & .065 & Dolerite ............. & Eskdale, Scotland............. & \\
\hline 58.63 & $.079(58.9)$ & Diorite........ & Unalaska Island.. & Bull. 168, 226.-W. T. \\
\hline 58.46 & $.083(58.7)$ & $\begin{array}{l}\text { Average of } 397 \text { igne- } \\
\text { ous rooks. }\end{array}$ & Grent Britılin ........... & Geol. Mag., 1899, 220.-W. T. \\
\hline 58.08 & $.075(58.2)$ & Andesite.............. & $\begin{array}{l}\text { Near Lassen Peak, Tehama County, } \\
\text { Cal. }\end{array}$ & Bull. 168, 182.-W. T. \\
\hline 56.61 & .082 & .... do. & $\begin{array}{l}\text { Sepulehre Mountain, Yellowstone } \\
\text { Park. }\end{array}$ & Bull. 168, 90.-W. T. \\
\hline 56.28 & .065 & Diorite. & Electric Peak, Yellowstone Park..... & Bull, 168, 87.-W.T. \\
\hline 55.83 & $.069(55.9)$ & Andesite..... & $\begin{array}{l}\text { Sepulchre Mountain, Yellowstone } \\
\text { Park. }\end{array}$ & Bull. 168, 90.-W. T. \\
\hline
\end{tabular}

Subrang 5. Plackrose.

(II. 4, 3, 5.)

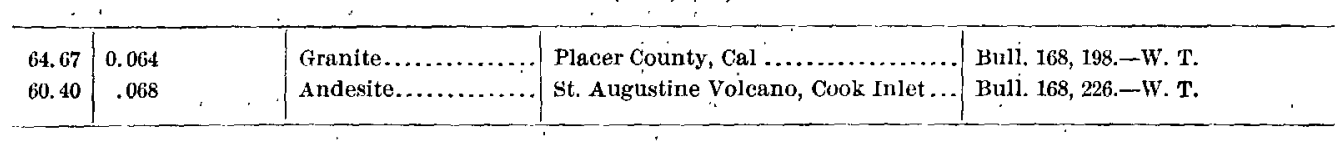

Rang 4. Bandase.

Subrang 1. Sagamose.

(II. $4,4,1$.)

\begin{tabular}{l|l|l|l|l}
\hline 55.48 & 0.023 & Tonalite ............. & Hokizawa, Sagami, Japan........... & W. T. \\
\hline
\end{tabular}

Subrang 3. Bandose.

(II. $4,4,3$.)

\begin{tabular}{|c|c|c|c|c|}
\hline 61.58 & 0.057 & Diorite. & Kadiak Island, Alaska .... & Bull. 168, 227.-W. T. \\
\hline 59.66 & .052 & Andesite ...... & Bandai San, Japan ... & W. T. \\
\hline 56.74 & .047 & Diabuse-porphyry. & Mount Morrison, Colo. . & Bull, 168, 141.-W. T. \\
\hline 56.51 & $.068(56.7-.0660)$ & Quartz-bisalt... & Near Lassen Peak, Cal. & Bull. 168, 185.-W. T. \\
\hline 56.41 & $.052^{\circ}$ & Diorite ... & Georgetown, D. C....... & Bull. 168, 44.-W. T. \\
\hline 56.31 & .044 & "Schliere" in granite & Riesengebirge, Silesia.. & N. J., 1898, XIT, 235.-W. T. \\
\hline 55.97 & .052 & Diorite ........... & Triadelphia, Md....... & Bull. 168, 44.-W. T. \\
\hline
\end{tabular}

Rane 5.

SUBRANG $3 . \longrightarrow$.

(II. 4, 5, 3.)

\begin{tabular}{l|l|l|l|l|}
\hline 51.62 & 0.015 & Diorite ............... & Kagelholmsön, Sweden .............. R. T., 1879, XXXII. \\
\hline
\end{tabular}


POOK ANALYSES USED IN CONSTRUCTING DIAGRAMS.

Classified list of analyses used in constructing diagrams-Continued.

Order 5. Germanarte.

Rang 1. Unytekase.

Subrang 2. Highwoodose.

(II. $5,1,2$.)

\begin{tabular}{|c|c|c|c|c|}
\hline $\begin{array}{c}\text { Silica } \\
\text { con- } \\
\text { tent. }\end{array}$ & $\begin{array}{c}\text { Alkali-silica } \\
\text { ratio. }\end{array}$ & Author's name. & Locality. & Referencé. \\
\hline 61.28 & 0.126 & Syenite........ & Tuolumne County, Cal ...... & Bull. 168, 204.-W. T. \\
\hline 58.04 & .166 & Trachyte.......... & Highwood Mountains, Mont . & Bull, 168, 131.-W. T. \\
\hline
\end{tabular}

(II. 5, 1, 3.)

\begin{tabular}{|c|c|c|c|c|}
\hline 62.99 & 0.139 & Umptekite & Beverly. Mass.. & T. M. P. MI., XX, 248.-W. 'T. \\
\hline 57.91 & .181 & Sodalite-trachyte.. & Monte Santo, Naples, Italy........... & A. J. S., VIII, 1899, 290.-W. T. \\
\hline 55.26 & .171 & Leucite-trachyte. & Viterbo, Italy $\ldots . . . \ldots \ldots \ldots$ & J. G., V, $370 .-W$. T. \\
\hline
\end{tabular}

SUBRANG 4. UMPTEKOSE.

(II. $5,1,4$.

\begin{tabular}{|c|c|c|c|c|}
\hline 64.28 & 0.156 & Sölvsbergite. & Andrews Point, Mrass. & J. G., VII, 481.-W. T. \\
\hline 63.71 & .152 & Umptekite ... & Koln, Finland ........ & Rosenbusch, El., 112.-W.T. \\
\hline 62.70 & .162 & Sölvsbergite.. & Laugendal, Norway ...... & E. K., I, 199.-W. T. \\
\hline 60.50 &, 159 & Eedrumite... & Ostö, Norway .......... & E. K., III, 37T.-W. T. \\
\hline 59.66 & .180 & Nephelite-syenite. & Crazy Mountains, Mont ........ & Bull. 168, 123.-W. T. \\
\hline 59.01 & .171 & .... do .......... & Red Hill, N. H & Bull. 168, 23.-W. T. \\
\hline 58.90 & .181 & $\begin{array}{l}\text { Nephelite-sülvsberg- } \\
\text { jte. }\end{array}$ & Aklungen, Norway... & E. K., I, 199.--W. T. \\
\hline 58.81 & .163 & Soda-syenite.... & Laupstadeid, Norway & B. M. A., 1898, VII, 48.-W. T. \\
\hline 58,46 & .162 & Nordmarkite.. & Cabo Frio, Brazil ...... & T. M. P. M.. XX, 244.-W. T. \\
\hline 57.52 & .172 & Iedrumite... & Brathagan, Norway..... & E. K., III, 190.-W. T. \\
\hline 57.00 & .170 & Heumite.... & .... do do .................. & E. K., III, 116.-W. T. \\
\hline
\end{tabular}

Rang 2. Monzonase.

Subrang 2. Ciminose.

(II. $5,2,2$.)

\begin{tabular}{|c|c|c|}
\hline 56.39 & $0.110(56.6)$ & Mica-trachyte....... \\
\hline 55.85 & .157 & Lencite-phonolite... \\
\hline 55.46 & .107 & Ciminite..... \\
\hline 55.21 & .151 & Leucite-trachyte.. \\
\hline 51.05 & .125 & Durbuchite..... \\
\hline
\end{tabular}

Monte Catini, Italy ............... A. J. S., IX, 1900, 47.-W. T

Bolseng, Italy ..................... J. G., V, 370.-W. 'I'.

Monte Cimino, Italy ................ A. J. S., IX, 1900, 44.-W. T.

Viterbo, Italy ...................... J. G., V, 370.-W. ']'

Schwarzwald, Baden............... W. T.

SUBRANG 3. MONZONOSE.

(II. $5,2,3$.

\begin{tabular}{|c|c|c|c|c|}
\hline 61.65 & 0.118 & Syenite. & $\begin{array}{l}\text { Yogo Peak, Little Belt Mountain's, } \\
\text { Mfont. }\end{array}$ & Bull. 168, 125.-W. T. \\
\hline 60.56 &. .127 & Granit & Elliott County, $\mathrm{Ky}$ & Bull. 168, 56.-W. T. \\
\hline 59.78 & .140 & Syenite.. & Custer County, Coln. . . . . . . . . . . . & Bull. 168, 151.-W. T. \\
\hline 58.18 & .134 & Kersantite. & Tito, Chile.... & Z. D. G. G., LI, 4.-W. T \\
\hline
\end{tabular}


Classified list of analyses used in constructing diagrams-Continued.

Subrang 3. Moxizonose-Continued.

\begin{tabular}{|c|c|c|c|c|}
\hline $\begin{array}{l}\text { Silica } \\
\text { con- } \\
\text { tent. }\end{array}$ & $\begin{array}{l}\text { Alkall-silica } \\
\text { ratio. }\end{array}$ & Author's name. & Locality. & Reference. \\
\hline 58.00 & 0.142 & Pulaskite. & Laugenthal, Norway ....... & E. K., III, 375.-W. T. \\
\hline 57.97 & .116 & Diorite... & Mount Ascutney, Vermont ........... & Büll. 168, 25.-W. T. \\
\hline 57.73 & .147 & Trachyte... & Ischia, Italy ............... & R. T., 1873, XXXVIII. \\
\hline 57.48 & .118 & Andesite ... & Pikes Peak district, Colo ........... & Bull. $168,145 .-W . T$. \\
\hline 57.29 & .134 & Quartz-banakite. & Head of Ishawooa River, Wyo & Bull. 168, 102.-W. T. \\
\hline 56.75 & .149 & Ciminite......... & 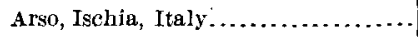 & A. J. S., VIII, $1899,290 .-$ W. T. \\
\hline 55.35 & .100 & Mica-basalt.... & Santa Maria Basin; Arizona... & Bull. 168, 173. -W. T. \\
\hline 55.23 & .143 & Trachyte.... & Highwood Mountains, Mont....... & Bull. 168, 131.-W.T. \\
\hline 54.86 & .114 & Shoshonite.. & Indian Peak, Wyo......... & Bull. 168, 100.-W.T. \\
\hline 54.42 & $.109(54.0)$ & Monzonite... & Yogo Peak, Mont....... & Bull. $168 ; 128 .-W$. T. \\
\hline 54.20 & .106 & ..... do .......... & Monzoni, Tyrol............ & E. K., II, 24.-W. T. \\
\hline 52.81 & $.124(52.6)$ & .... do $\ldots \ldots \ldots \ldots$ & Bearpaw Mountains, Mont........... & Bull. 168, 135.-W. T. \\
\hline 52.80 & .150 & Shonkinite-monzonite & Máros, Celebes ............. & G. C., 25. \\
\hline 52.63 & .133 & Banakite ...... & Hoodoo Mountain, Wyo.... !. & Bull. 168, 101.-W. T. \\
\hline 52.33 & .147 & ....do.... & Head of Ishawooa River, Wyo. & Do. \\
\hline 52.14 & .116 & Syenite.... & Heidelberg, Baden ................. & R. T., 1879, XXIV. \\
\hline 51.82 & .134 & Banakite... & $\begin{array}{l}\text { Head of Lamar River, Yellowstone } \\
\text { Park. }\end{array}$ & Bull. 168, 101.-W. T. \\
\hline 51.46 & .181 & do & Ishawooa Canyon, Wyo .... & Do. \\
\hline
\end{tabular}

Subrang 4. AK EROSE.

(II. 5, 2, 4.)

\begin{tabular}{|c|c|c|c|c|}
\hline 61.87 & 0.119 & Syenite... & Custle Mountnin district, Mont....... & Bull. 168, 130.-W. T. \\
\hline 61.63 & .125 & Nephelite-syenite & Mulatto, Tyrol ....... & K. A. W. W, $\mathrm{CXI}, 1,260$ \\
\hline 61.26 & .104 & Andesite .... & Colombia, South America.. & J. G., I, 171.-W. T. \\
\hline 61.08 & .114 & Porphyrite ...... & Crazy Mountains, Mont.... & Bull. 168, 120. -W. T. \\
\hline 60.44 & .110 & Diorite-porphyry ..... & La Plata Mountains, Colo.. & Bull. 168, 162.-W. T. \\
\hline 59.56 & .125 & A kerite........ & Vettakollen, Norway........ & Z, K., XVI, 50.-W. T. \\
\hline 58.28 & .155 & Syenite... & Crazy Mountains, Mont... & Bult. 168, 121.-W. T: \\
\hline 57.01 & .124 & Andesite ........ & Silver Cliff, Colo........... & Bull. $168,148 .-$ W. T. \\
\hline 56.79 & .118 & Akerite...... & Vettakolien, Norway.... & Z. K., XVI, 50.-W. T, \\
\hline 56.75 & .108 & Porphyrite.. & Crazy Mountains, Mont .... & Bull. 168, 121.-W. T. \\
\hline 56.02 & .119 & Andesite : & Taal, Luzon, P. I.... & N. J., B. B., I, 1881, 482. \\
\hline 55.95 & .135 & Ditroite ........ & Nosy Komba, Madagascar... & M. M., 204. \\
\hline 55.53 & $\ldots 115$ & Diorite ........ & La Plata Mountains, Colo.. & Bull. 168, 162.-W. T. \\
\hline 54.69 & $.136(54.6)$ & Porphyrite ... & Crazy Mountains, Mont .... & Bull. 168, 121.-W. T. \\
\hline 53.80 & .144 & Diorite ..... & Silver Cliff, Colo............. & Bull. 168, 147.-W. T. \\
\hline 53.60 & .124 & Basalt .... & Reinhardswald, Prussia .......... & W. T. \\
\hline 53.15 & .129 & Essexite.... & Shefford Mountain, Quebec ..... & J. G., XI, 265. \\
\hline 53.12 & .171 & Tephritic-trachyte - & Columbretes Islands ...... & T. M. P. M., XVI, 314.-W. T. \\
\hline 51.42 & $.130(51.8)$ & Basalt ... & S. Rhone .............. & R. T., 1879, LXXII. \\
\hline 51.22 & .162 & Minette . & Brathagen, Norway .... & E. K., III, 376.-W T \\
\hline
\end{tabular}

RANG. 3. ANDASE.

SUBRANG 2: -

(II. $5,8,2$.)

\begin{tabular}{l|l|l|l|l|}
\hline 57.31 & 0.094 & Ciminite ............ Monte Cimino, Italy ................ A. J. S., JX, 1900, 44.,W. T. \\
\hline
\end{tabular}


Classified list of analyses used in constructing diagrams--Continued.

SURHANG S. SHOSHONOSE.

(II, 5, 3, 3.)

\begin{tabular}{|c|c|c|c|c|}
\hline $\begin{array}{l}\text { Silica } \\
\text { con- } \\
\text { tent. }\end{array}$ & $\begin{array}{l}\text { Alkali-silica } \\
\text { ratio. }\end{array}$ & Author's name. & Locality. & Reference. \\
\hline 59.41 & 0.102 & Andesite .... & Cabo da Gata, Spain.... & Z. D., g. G., XLIII, 719.-W. T. \\
\hline 38.51 & $.095(58.8)$ & Mica-diorite. & Crystals Falls, Mich ........... & Bül. 168, 67.-W.T. \\
\hline 57.97 & $.083^{\cdot}(57.8)$ & Diorite ..... & Crazy Mountains, Mont ......... & Bull. 168, 122.-W. T. \\
\hline 56.90 & .097 & Syenite.... & Denver Basin, Colo................. & Bull, 168, 140.-W. T. \\
\hline 56.78 & .093 & Latite $. . . \ldots \ldots . .$. & Clover Meadow, Cal ................ & Bull. 168. 205.-W. T. \\
\hline 56.19 & .092 & .... do do....... & $\begin{array}{l}\text { Table Mountain, Tuolumne County, } \\
\text { Cal. }\end{array}$ & Bull. 168, 205.-W. T' \\
\hline 56.05 & .107 & Shoshonite. & Two Ocean Pass, Yellowstone Park.. & Bull. 168, 100.-W. T. \\
\hline 55.69 & $.100(55.6)$ & Vulsinite... & Rocea Monfina, Italy ............... & J. G., V, 358.-W. T. \\
\hline 54.56 & $.076(.075)$ & Andesite ........ & Radicofani, Italy . . . . . . . . . . . . & A. J. S., IX, 1900, 52.-W. T. \\
\hline 54.14 & .077 & $\ldots$. do ........ & $\ldots$ do $\ldots \ldots \ldots \ldots$. & Do. \\
\hline 53.49 & .104 & Shoshonite... & Beaverdam Creek, Yellowstone Park. & Bull. 168, 100.-W. T. \\
\hline 52.93 & .132 & Banakite ............. & $\ldots \ldots$ do $\ldots \ldots \ldots \ldots \ldots \ldots \ldots \ldots \ldots \ldots \ldots$ & Bull. 168, 102.-W. 'T.' \\
\hline 52.59 & $.099(52.7)$ & Basalt ............ & Table Mountain, Colo............... & Bull. 168, 140.-W. T: \\
\hline 52.49 & .102 & Shóshonite. & Pyramid Peak, Yellowstone Park.... & Bull. 168, 100.--W. T. \\
\hline 52.33 & .103 & Augite-porphyy & Cottonwood Creek, Mont............ & Bull. 168, 112.-W. T. \\
\hline 52.11 & $.097(51.8)$ & Basalt ....... & Crandall Basin, wyo......... & Bull. 168, 92.-W. T. \\
\hline 51.00 & .115 & Monzonite....... & Highwood Mountains, Mont......... & Bull. 168, 133.-W. T. \\
\hline 50.98 & $.120(50.7)$ & Mondhaldeite.. & Mondhalde, Kaisérstuhl, Baden & W. T. \\
\hline 50.08 & .137 & $\ldots$. do ...... & $\ldots \ldots$ do $\ldots \ldots \ldots \ldots \ldots \ldots \ldots$ & Do. \\
\hline 50.06 & .110 & Shoshonite.. & Lamar River, Yellowstone Park & Bull. 168, 100.-W. T. \\
\hline 49. 69 & .107 & Basalt ....... & Denver Basin, Colo................. & Bull. 168, 140.-W. T. \\
\hline 48.25 & .118 & Dolerite... & .....do...$\ldots \ldots \ldots \ldots \ldots \ldots$. & Do. \\
\hline 47.50 & .117 & Labrador-porphyrite. & Gran; Norway ............... & Q.J. G. S., L, 83.-W. T. \\
\hline 45.53 & .140 & Leucite-monchiquite. & Bohemian Mittelgebirge. & Rosenbusch $_{b} \mathrm{El}$, 235. $^{-}$W. T. \\
\hline
\end{tabular}

SUBRANG 4. ANDOSE.

(II. $5,3,4$ )

\begin{tabular}{|c|c|c|c|c|}
\hline 58.45 & 0.080 & Diorite.......... & Pen Maen Mawr, N. Wales...... & Q.J. G. S., XXXIII, 424. \\
\hline 58.42 & .099 & Andesite........ & 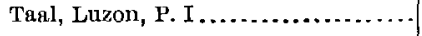 & N. J., B. B., I, 1881, 481. \\
\hline 58.05 & .083 & Diorite - & Electric Peak, Yellowstone Park.. & Bull. 168,87.-W.T. \\
\hline 57.64 & $.104(57.8)$ & Augite-porphyrite . & Crandall Basin, Wyo ............. & Bull. 168, 93.-W. 'Г. \\
\hline 57.59 & $.070(57.8)$ & Quartz-basalt... & Silver Lake, Lassen, Cal....... & Bull: $168,185 .-W . T$. \\
\hline 57. 38 & $.071(57.1)$ & Pyroxene porphyry. & Electric Peak, Yellowstone Park. . & Bull, 168, 87.,-W. T. \\
\hline 57.32 & $.098(57.5)$ & Monzonite..... & Crandall Basin, Wyo .............. & Bull. 168, 93. -W. 'T'. \\
\hline 57,26 & .102 & Diorite ........ & $\ldots$ do $\ldots \ldots$. $\ldots \ldots \ldots \ldots \ldots$ & Do. \\
\hline 57.25 & .067 & Quurtz-basalt. & Cinder Cone, Lassen, Cal........ & Bull. 168, 184.-W. T. \\
\hline 57.17 & .079 & Andesite............. & $\begin{array}{l}\text { Sepulchre Mountain, Yellowstone } \\
\text { Park. }\end{array}$ & Bull. $168,90 .-W . T$ \\
\hline 57.11 & $.084(57.5)$ & $\ldots$. . do ... & Mill Creek, Lassen, Cal........... & Bull. 168, 182.-W. 'I'. \\
\hline 56.91 & .085 & ..... do. & Colombia, South America... & J. G., I, 171.-W. T. \\
\hline $56.70^{-}$ & $.074(56.6)$ & Quartz-basalt.. & Cinder Cone, Lassen, Cal ......... & J. G., I, 184.-W.T. \\
\hline 56.49 & $.112(57.1)$ & Diorite.. & Little Falls, Minn . . . . . . . . . . . & R. T., 1879, XXXIV. \\
\hline 56.21 & .104 & Gabbro-diorite..... & Crandall Basin, Wyo.................... & Bull. 168, 98.-W. T. \\
\hline 56.19 & .076 & Andesite ...... & Buffalo Peaks, Colo $\ldots . . . . . . . . . . .$. & Bull. 168, 153.-W. T. \\
\hline 56.09 & .080 & Diorite .... & Campo Major, Portugal ........ & E. K., II, 37.-W. T. \\
\hline 56.07 & $.091(55.9)$ & Andesite. & Bogoslof Island ........... & Bull. 168, 227.-W. T. \\
\hline 55.93 & $.103(55.8)$ & Gabbro-diorite ....... & Crandall Basin, Wyo ................ & Bull. 168, 93.-W. T. \\
\hline 55.92 & .095 & Andesite ........ & $\begin{array}{l}\text { Sepulchre Mountain, Yellowstone } \\
\text { Purk. }\end{array}$ & Bull. 168, 90.-W. T. \\
\hline 55.80 & .076 & Diorite (norite) & Klausen, Tyrol ... & E. K., II, 37.-W. T. \\
\hline 55.34 & .093 & Norite ............. & Montrose Point, N. Y... & W.T. \\
\hline
\end{tabular}


Classifiedl list of analyses used in comstrucling diagrams-Continned.

Subrang 4. ANDose-Continued.

\begin{tabular}{|c|c|c|c|c|}
\hline $\begin{array}{l}\text { Silicg } \\
\text { con- } \\
\text { tent. }\end{array}$ & $\begin{array}{c}\text { Alkali-silica } \\
\text { ratio. }\end{array}$ & Author's name. & Locality. & Reference. \\
\hline .55 .20 & $0.075(55.4)$ & Andesite. & Butte County, Cal & Bull. 168, 182.-W. T. \\
\hline 55.13 & .107 & Diorite ....... & Neihurt, Mont ..................... & Bull. 168, 127.,W. I. \\
\hline 54.62 & .075 & Andesite .... & Iuzon, P.I..................... & N. J., B. B., I, 1881, 471. \\
\hline 54,50 & .108 & Diorite-porphyrite. & Crazy Mountuins, Mont .. & Bull. 168, 121.-W.T. \\
\hline 54.52 & .092 & Diabase ....... & Diabase Hills, Nev............. & Fortieth Pur., II, 812. \\
\hline 53.94 & .086 & Dolerite ....... & …. do $\ldots \ldots \ldots \ldots$ & Do. \\
\hline 68.89 & .099 & Basic andesitic glask.. & Indian Ridge, Wyo ...... & BulI.168, 92.-W.T. \\
\hline 53.71 & .098 & Micn-gabbro.......... & Crandall Basin, Wyo .............. & Do. \\
\hline 53.48 & $.089 \cdot-$. & Quartz-diorite....... & Crazy Mountains, Mont ............. & Bull. 168, 121.-W. T. \\
\hline 53.10 & $.134(53.2)$ & Covite........ & Nosey Komba, Madagascar... & M. M., 204. \\
\hline 52.97 & $107 \quad$ & Busalt ............... & Pikes Peak district, Colo .......... & Bull. 168, 145.-W. T. \\
\hline 52.91 & .119 & $\begin{array}{l}\text { Diabase-syenite-por- } \\
\text { phyry. }\end{array}$ & Holmestrand, Norway ................. & Z.K., XVI, 28.-W.T. \\
\hline 52.63 & $.082(52.7)$ & Busalt ... & Shusta County, Cal .... & Bull. 168;180.-W.T. \\
\hline 52.38 & .093 & ..... do ..... & Rio Grande, N.Mex ... & Bull. 168, 169.-W.T. \\
\hline 52.37 & .084 & ..... do .... & Absaroka Range, Yellowstone Park. & Bull. 168, 109.-W.T. \\
\hline 52.37 & $.082(51.8)$ & $\ldots \ldots d o_{1}$. & Rio Grande, N. Mex .. & Bull. 168, 169.-W.T. \\
\hline 52.27 & .095 & .....do..... & .... do ............................ & Do. \\
\hline 52.12 & .095 & Diorite & Mount Ascutney, Vt............... & Bull. 168,25.-W.T. \\
\hline 52.09 & .085 & Busalt,.. & Crandall Basin, Wyo .............. & Bull. 168, 92.-W. T. \\
\hline 51.57 & .090 & $\because \ldots$ do $\ldots$ & Rio Grande, $N$, Mex $\ldots \ldots \ldots . . . . .$. & Bull. 168, 169.-W.T. \\
\hline 51.54 & .110 & Andesite ...... & Bogoslof Island, Alaska ................ & Bull. 168, 227.--W.'T. \\
\hline 50.99 & $.101:$ & Camptonite :... & Ishawooa Canyon, Wyo .............. & Bull. 168, 110.-W. T. \\
\hline 50.97 & $.112(50.6)$ & Kersantite........ & Guanta, Chile...$\ldots \ldots \ldots \ldots \ldots$ & Z.D.g.G., LI, 4 . \\
\hline 60.73 & .099 & Diorite ......... & Crazy Mountains, Mont ................. & Bull. 168, 122.-W. T. \\
\hline 50.56 & .079 & Basalt .......... & Plumas County, Cal ............ & Bull. 163, 189.-W. T. \\
\hline 49.40 & .087 & Facies of monzonite.. & Predazzo, Tyrol .................. & E. K., II, 102.-W. T. \\
\hline 49.24 & $.092(49.7)$ & Dolerite.... & Ferdinandea ......... & T. М. P. M., 1888, 398. \\
\hline 49.04 & .110 & Basalt .......... & Buffalo Peak, Colo'.......... & Fortieth Par., I, 676. \\
\hline 48.90 & .090 & Diorite $\ldots \ldots$ & 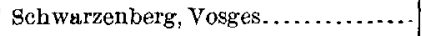 & Rosenbusch, El., 140. \\
\hline 48.85 & .131 & Essexite ........ & Mount Johnison, Quebec..... & J. G., XI, 265. \\
\hline 48.76 & .105 & Basalt ....... & Pikes Peak district, Colo... & Bull: 168, 145.-W. T. \\
\hline 48.18 & .093 & Olivine-diabase ...... & Biclla, Piedmont ................. & R. T., 1884, XLIT. \\
\hline 48.06 & .084 & Kersantite............ & Laugendal, Norway ................. & E. K., III, 376,一W. T. \\
\hline 44.85 & .110 & Nephelite-tephrite ... & Mittelgebirge, Bohemia ..... & Rosenbusch, El., 346.-W. T, \\
\hline
\end{tabular}

(II. $5,3,5$.

\begin{tabular}{|c|c|c|c|c|}
\hline 55.08 & 0.081 & Andesite-basalt. & Shasta County, Cal .......... & Bull, 168, 176.-W. T. \\
\hline 52,58 & .073 & Andesite. & S. W. Tillis......... & Z. D. g. G., XXTX, 823. \\
\hline 47. 21 & .109 & Beerbachite.. & Odenwald, Hesse........................ & Rosonbusch, El., 219.-W. T. \\
\hline
\end{tabular}

RANG 4. Hegsase.

Subrang 3. Hésose.

(II. $5,4,3$.

\begin{tabular}{|c|c|c|c|c|}
\hline 57.47 & 0.072 & Basalt . & Cascade Range, Oreg..... & Bull. 168, 223.-W. T. \\
\hline 56.94 & .074 & Anorthosite. & 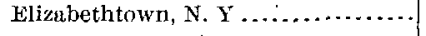 & Bull. 168, 37.-W. T. \\
\hline 55.53 & .062 & Andesite. & Plumas County, Cal ................. & Bull. 168, 181.-W. T. \\
\hline 55.14 & .075 & Secretion in dacite & Lassen Peak, Cal.......... & Bull. 168, 179.-W. T. \\
\hline
\end{tabular}


Classified list of analyses used in constructing diagrams-Continued.

Rang 4. Hessase-Continued.

Subrasg 3. Hessose-Continued.

\begin{tabular}{|c|c|c|c|c|}
\hline $\begin{array}{l}\text { Silica } \\
\text { con- } \\
\text { tent. }\end{array}$ & $\begin{array}{l}\text { Alkali-silica } \\
\text { ratio. }\end{array}$ & Author's name. & Locality. & Reference. \\
\hline 53.91 & 0.066 & Dolerite.. & Plumas County, Cal. & Bull. 168, 189.-W. T. \\
\hline 53.35 & .063 & Secretion in dacite... & Lassen Peak, Cal . . . . . . . . . . & Bull. 168, 180.-W. T, \\
\hline $53.18:$ & .082 & Anorthosite. & $\begin{array}{l}\text { Whiteface-Mountain; Adirondacks, } \\
\text { N.Y. }\end{array}$ & Bnll, 168, 36.-W. T: \\
\hline 53.00 & .070 & Diorite. & Schwarzenburg, vosges ............. & Rosenbusch, El, 140. \\
\hline 52.95 & $.073(52.7)$ & Basalt ..... & Shasta County, Cal. & Bull. 168, 186.-W. T. \\
\hline 52.10 & .074 & Monzonite..... & Predazzo, Tyrol .... & E. K., II, 25.-W. T. \\
\hline 52.05 & $.075(51.7)$ & Diorite... & Ouray County, Colo & Bull. 168, 161.-W.T. \\
\hline 51.27 & .064 & ..... do $\ldots . . . .$. & Little Falls, Minn ... & R. T, 1879, XXXIV. \\
\hline 49.93 & .074 & Dolerite .. & Paterno, Etria, Sicily ....... & R. T., 1884, LXXX. \\
\hline 49.88 & .057 & Olivine-gabbro. & Pigeon Point, Minn ........... & Bull. 168, 76.-W. T. \\
\hline 49.80 & .049 & Gabbro.... & Crystul Falls, Mich.. & Bul1. $168,67 .-W . T$. \\
\hline 49.15 & .095 & ..... do ... & Duluth, Minn ....... & Rosenbusch, El., 151. \\
\hline 48.29 & .039 & Gabbro diorite .. & Minnesota Falls, Minn .. & Bull. 168, 83.-W. T. \\
\hline 47.94 & $.060(47.5)$ & Basalt ............. & Shasta County, Cal ........... & Bull. 168, 176.-W. T. \\
\hline 47.88 & .065 & Gabbro.... & Elizabethtown, N. Y........ & Bull. 168, 37.-W. T. \\
\hline 46.71 & .036 & Olivine-gubbro.... & Langenlois, Austria ....... & R. T., 1879, XXXVIII. \\
\hline 46.45 & .047 & $\ldots . . . . .$. & Minnesota.................. & Bull. 168, 82,-W. T. \\
\hline 45.20 & .071 & Diorite .......... & Tuc d'Ess,'Pyrenees...... & C. G. I., VIII, 1901, 832.-W. T, \\
\hline 44.72 & .047 & Corsite ........... & Poudière, Auvergne.. & R. T., 1879, XXXVI. \\
\hline 43.42 & .044 & Gabbro-diorite. . & Ilehester, Ma....... & Bull. $168,44 .-\mathrm{W} . \mathrm{T}$. \\
\hline 43.19 & .094 & Basalt ......... & Westphalia ........... & R. T., 1879, LXXIV. \\
\hline
\end{tabular}

Rang 5. Corsase.

(II, 5, 5.)

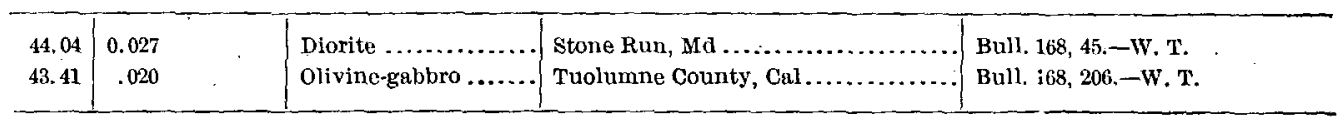

Order 6. Norgare.

Rang 1. Laurdalase:

Subrang 2. Fergusose.

$\therefore \quad$ (II, 6, 1, 2.)

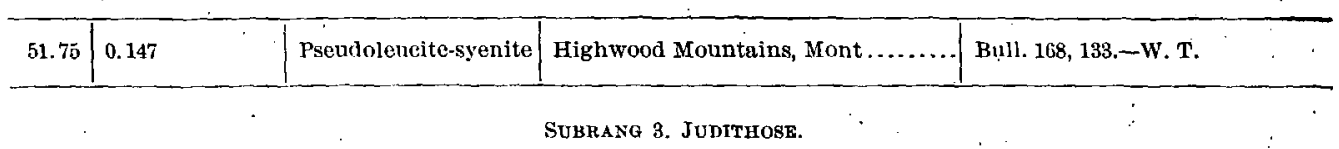

(II.' 6, 1, 3.)

\begin{tabular}{|c|c|c|c|c|}
\hline 57.63 & 0.198 & Tinguaite & Judith Mountains, Mont ............. & A. J.S., II, 1896, 192.-W. T. \\
\hline $5 \pi .46$ & $.196(57.3)$ & ..... do .... & Bearpaw Mountains, Mont............ & Bull. 168, 136.-W. T. \\
\hline 53.09 & $.226(53.2)$ & Foyaite....... & Magnet Cove, Ark........... & J. G., $1 X, 611 .-W$. T. \\
\hline 52.91 & .224 & Leucite-tinguaite .... & $\ldots \ldots$ do $\ldots . . . . . . . . .$. & I. R. A., 287.-W. T. \\
\hline 51.94 & .157 & Trachyte..... & Highwood Mountrins, Mont. & Bull. 168, 131. -W. T. \\
\hline
\end{tabular}


Classified list of analyses used in constructing diagrams-Continued.

SUbrang 4. LaURdalose.

(II. 6, 1, 4.)

\begin{tabular}{|c|c|c|c|c|}
\hline $\begin{array}{l}\text { Silíca } \\
\text { con- } \\
\text { tent. }\end{array}$ & $\begin{array}{l}\text { Alkali-siliea } \\
\text { ratio. }\end{array}$ & Author's name. & Locality. & Reference. \\
\hline 59.88 & 0.188 & Hedrumite. & Hedrum, Norway. & E. K., III, 377.-W. T. \\
\hline 56.58 & .242 & Tinguaite.. & .... do .................. & E. K., I, 199,-W. T. \\
\hline 56.35 & .211 & Laurdalite .. & Farris, Norway ............... & E. K., III, 375.-W. T. \\
\hline 55.90 & .209 & Tinguaite ... & Foya, Portugal .............. & T. M. P. M., XVI, 262.-W. T. \\
\hline 55.65 & .225 & .....do ..... & Hedrum, Norway . ............ & E. K., I, 199.-W. T. \\
\hline 55.18 & .182 & Syenite..... & $\ldots$. do $\ldots . . . . . . .$. & E. K., III, 375.-W. T. \\
\hline 54.55 & .192 & Luurdalite..... & Lougendal, Norway ........... & Do. \\
\hline 54.04 & .233 & Leucite-tinguaite... & Magnet Cove, Ark..... & I. R. A., 287.-W. T. \\
\hline 53.81 & .192 & Syenite-pegmatite... & Stoksund, Norway......... & Z. K., XVI, 116.-W. T. \\
\hline 53.73 & .214 & Nephelite-syenite .... & Transvaal .......... & Rosenbusch, E]., 126.-W. T. \\
\hline 61.95 & $.155(51.6)$ & Soda-minette........ & Langesundsfjord, Norway ........... & E. K., II, 376.-W. T. \\
\hline 50.96 & .229 & $\begin{array}{l}\text { Leucite-n ephelite- } \\
\text { syenite. }\end{array}$ & Magnet Cove, Ark........... & I. R. A., 276.-W.T. \\
\hline 47.61 & .190 & Tinguaite ........ & Two Buttes, Colo..... & Bull, $168,165 .-W . T$. \\
\hline 45.16 & .195 & Olivine-laurdalite.... & Furris, Norway ........... & E. K., III, 375.-W. T. \\
\hline
\end{tabular}

Rang 2. Essexase.

Subrang 3. Borolanose.

(II. 6, 2, 8.)

\begin{tabular}{|c|c|c|}
\hline 52.05 & 0.132 & Monzonite. \\
\hline 51.35 & .165 & Nephelite-felsite. \\
\hline 50,24 & $.153(49.9-.154)$ & Leucite-tephrite. . \\
\hline 50.15 & $.142(49.8)$ & Shonkinite ....... \\
\hline-49.70 & .167 & Covite... \\
\hline 47.8 & .204 & Borolanite.. \\
\hline
\end{tabular}

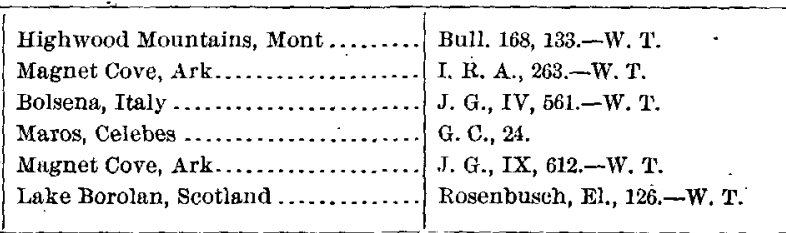

Subrang 4. Lissexose.

(II. $6,2,4$.

\begin{tabular}{|c|c|c|c|c|}
\hline 55.07 & 0.162 & Rhombenporphyry. & Stoksund, Norway .. & Z. K., XVI, 116-W. T. \\
\hline 54.34 & .173 & Nephelite-syenite. & Cripple Creek, Colo. & Bull. 168, 144.-W. T. \\
\hline 51.90 & .210 & Laurdalite.... & Lunde, Norway .......... & E. K., III, 375.-W. T. \\
\hline 51.10 & .173 & Covite.... & Nosy Komba, Madagascar.. & M. M., 204. \\
\hline 50.50 & .143 & Nephelite-monzonite. & Rongstock, Bohemir....... & T. M. P. M., XIV, 98, 99.-W. T. \\
\hline 50.26 & .210 & $\begin{array}{l}\text { Nephelite-sycenite- } \\
\text { porphyry. }\end{array}$ & Viezzena, Tyrol ......... & K. A. W. W., CXI, I, 275 . \\
\hline 49.90 & $.155(49.3)$ & Leucite-kulaite....... & Kula, Asia Minor ....... & J. G., VIII, 613.-W. T. \\
\hline 48.69 & $.151(49.0-.150)$ & Olivine-essexite .. & Mount Johnson, Quebec .. & J. G., XI, 265. \\
\hline 48.46 & .243 & Heumite ........ & Longendal, Norway ....... & E. K., III, 376.-W. T. \\
\hline .48 .35 & .161 & Kulaite.... & Kula, Asia Minor ............. & J. G., VIII, 613.-W. T. \\
\hline 17. 94 & $.150 .(48.3)$ & Essexite ........ & Salem Neck, Mass . ................ & Rosenbusch, E1., 172. $-\mathrm{W}$ : T. \\
\hline 47.67 & $.150(48.0)$ & Theralite.... & Alabaugh Creek, Mont..... & Bull. 168, 124.-W. T. \\
\hline 46.48 & .162 & Monchiquite & Rio de Janeiro, Brazil... & Rosenbusch, El., 235.-W.T. \\
\hline
\end{tabular}


Classified list of analyses used in constructing diagrams-Continued.

Rang 3. Salemase.

Subrang 4. Salemose.

(II. 6, 3, 4.)

\begin{tabular}{|c|c|c|}
\hline $\begin{array}{l}\text { Silica } \\
\text { con- } \\
\text { tent. }\end{array}$ & $\begin{array}{l}\text { Alkali-silica } \\
\text { ratio. }\end{array}$ & Author's name. \\
\hline 50.47 & 0.132 & Augite-diorite $\ldots . .$. . \\
\hline 49.95 & .132 & Microessexite ....... \\
\hline 48.50 & .138 & Essexite .... \\
\hline 46.40 & .151 & Augite-diorite....... \\
\hline 45.32 & .110 & Hornblende-gabbro .. \\
\hline 43.66 & .142 & Essexite ............. \\
\hline
\end{tabular}

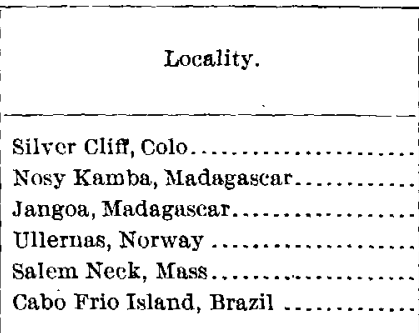

Reference.
Bull, 168, 147.-W. T.
M. M., 204.
Do.
Z. K., XVI, 49.-W. T.
J. G., VII, 63.-W. T.
Rosenbusch, El., 172.-W. T.

SuBRANG 5.

(II. 6, 3, 5.)

\begin{tabular}{l|l|l|l|l|}
\hline 47.90 & 0.137 & Basalt ............... & Pedregal, Mexico $\ldots \ldots \ldots \ldots \ldots \ldots \ldots .$. & W. T. \\
\hline
\end{tabular}

RANG 4.

Subrang 3.

(II. 6, 4, 3i)

\begin{tabular}{|c|c|c|c|c|}
\hline $\begin{array}{l}45.76 \\
45.11\end{array}$ & $\begin{array}{r}0.086 \\
.090\end{array}$ & $\begin{array}{l}\text { Gabbro } \ldots \ldots \ldots \ldots \ldots \\
\text { Diorite } \ldots \ldots \ldots \ldots \ldots\end{array}$ & 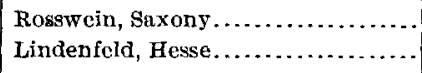 & $\begin{array}{l}\text { N. J., 1893, II, 503.-W. T. } \\
\text { W. T. }\end{array}$ \\
\hline
\end{tabular}

Orden 7. Italake.

Rang 1. LuJavrase.

SUBRANG 3. JANEIROBE.

$$
\text { (II. } 7, \text { I, 3.) }
$$

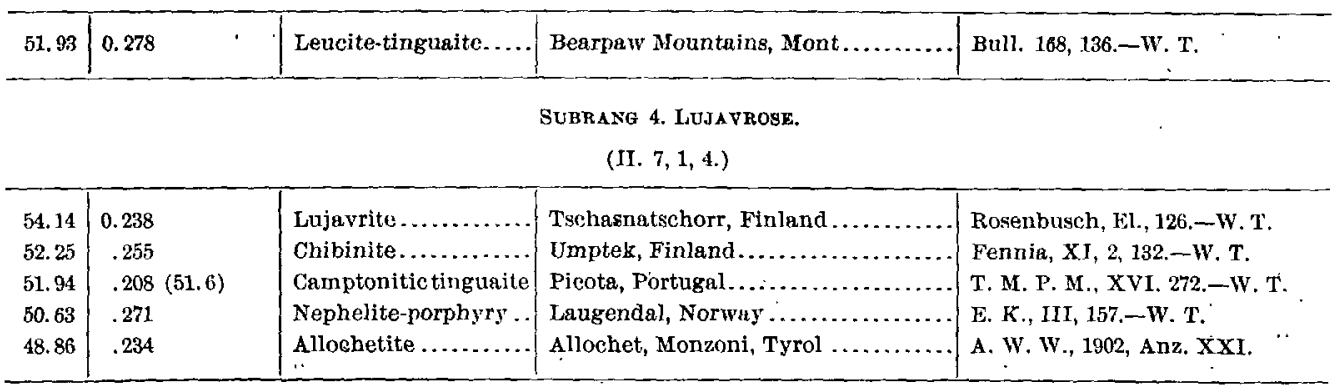

Rang 2. Vulturase.

SUBRANG 2. -

(II. $7,2,2$.

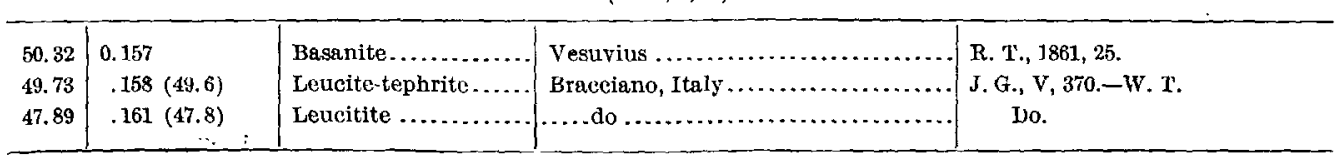


Classificd list of analyses used in constructing diagrams-Continued.

SUBRaNG 3.

(II. $7,2,8$ )

\begin{tabular}{|c|c|c|c|c|}
\hline $\begin{array}{l}\text { Silica } \\
\text { con- } \\
\text { tent. }\end{array}$ & $\begin{array}{l}\text { Alkuli-sirlica } \\
\text { ratio. }\end{array}$ & Author's name. & Locality. & Reference. \\
\hline 48.03 & 0.167 & B.sanite......... & 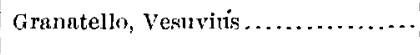 & R. T., 1861, 25 \\
\hline & 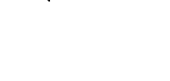 & , & $\begin{array}{l}\text { Subrang 4. VULTUHose. } \\
\text { (II. } 7,2,4 . \text { ) }\end{array}$ & \\
\hline \multirow[t]{2}{*}{42,46} & 0.229 & Haüynophyre........ & Melfi, Italy $\ldots \ldots \ldots \ldots \ldots \ldots \ldots \ldots \ldots$ & Rosenbusch, El., 857. \\
\hline & & & $\begin{array}{c}\text { RANG } 3 .-- \\
\text { SUBRANG } 3 .- \\
\text { (II. } 7,3,3 .)\end{array}$ & . \\
\hline 47.40 & 0.130 & Leucite-tephrite..... & Rocea Monfina, Italy ................ & J. G., V. 370.-W. T. \\
\hline
\end{tabular}

Order 8. Campanare.

RANG 1 .

SURRANG 3. -

(II. 8, 1, 3.)

\begin{tabular}{|c|c|c|c|c|}
\hline $\begin{array}{l}50.00 \\
46.48\end{array}$ & $\begin{array}{r}0.267 \\
268\end{array}$ & Tingunite ............. & $\begin{array}{l}\text { Beemerville, N.J........................... } \\
\text { Etinde Volcano, Kamerund, Africa. }\end{array}$ & $\begin{array}{l}\text { Bull. Nus. Comp. Zool.; XXXVIII, } \\
\text { 1902, 276.-W. T. } \\
\text { W. T. }\end{array}$ \\
\hline
\end{tabular}

SuRraNg 5. -

(II. $8,1,5$.

$47.43[0.383 . \quad$ Soda-sussexite........ $]$ Penikkavaara, Finland ............. B. C. G. F., XI, 22., W. T. .

\section{Rang 2. Vesuvasie.}

Subrang 2. VesUVose.

(IT. $8,2,2$. )

\begin{tabular}{|c|c|c|c|c|}
\hline 47. 71 & 0.157 & Leucite-basunite ..... & Lave of 1631 , Vesuvius...... & W. T. \\
\hline 47.65 & .157 & .... do .......... & Lavu of 1872, Vesuvius... & W. T. \\
\hline 47.53 & .149 & Iscucitophyre... & Vesuvius ......................... & R. T., 1884, LVI. \\
\hline 46.36 & $.160(46.2)$ & .....do ......... & 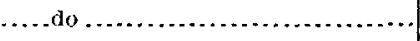 & R. T., 1884, LVIII. \\
\hline
\end{tabular}

\section{Order 9. Lappare.}

Rang 1. Urtase.

SURRANG 3. ARKANSOSE.

(II. 9, I, 3.)

\begin{tabular}{l|l|l|l|l|l}
\hline 44.40 & 0.252 & Arkite............... & Magnet Cove, Ark.................. J. G., IX, 616. - W. T. \\
\hline
\end{tabular}


ROCK ANALYSES USED IN CONSTRUCTING DIAGRAMS.

Classified list of analyses used in constructing diagrams-Continued.

Surraxg 4. URTOSE..

(II. 9, 1,4.)

\begin{tabular}{|c|c|c|c|c|}
\hline $\begin{array}{l}\text { Silica } \\
\text { con- } \\
\text { tent. }\end{array}$ & $\begin{array}{l}\text { Alkali-sillea } \\
\text { ratio. }\end{array}$ & Author's name. & Iocality. & Reference. \\
\hline 45.46 & 0.397 & Urtite $\ldots . . .$. & Lujıvr Urt, Kola, Finland ........ & G. F. S. F., XVIII, 462,-W. T." \\
\hline 45.43 & .391 & $\ldots . . . d 0 \ldots$. & 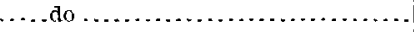 & Do. \\
\hline 45.28 & .418 & $\ldots$. do ......... & $\ldots \ldots$ do $\ldots \ldots \ldots \ldots \ldots$ & Rosenbusch, El, 126.-W, T. \\
\hline 43.02 & .378 & $\begin{array}{l}\text { Ijolite, rich in nephe- } \\
\text { lite. }\end{array}$ & Kuuosamo, Finland.. & B. C. G. F., XI, 17.-W. T. \\
\hline
\end{tabular}

CLASS III. SALFEMANE.

Order 3. Atllantare.

Rang 1. Rockaliase.

Subrang 2. -

(III. $3,1,2$.

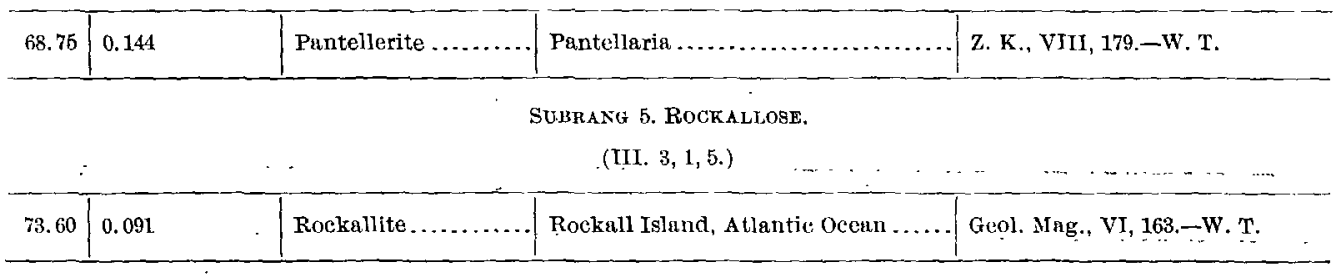

Order 4. Vaalare.

RANG 3. VAALASE.

Sublang 4. Vaatose.

(III. $4,3,4$ )

\begin{tabular}{|c|c|c|c|c|}
\hline 53.39 & 0.068 & Quartz-gabbro........ & 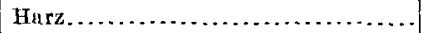 & R. T., 1884, XXXIV. \\
\hline 53.35 & $.067(52.8)$ & Basalt ............... & $\begin{array}{l}\text { Teánaway River, Kittitas County, } \\
\text { Wash. }\end{array}$ & Bull, 168, 225.-W. T. \\
\hline 52.67 & $.047(52.9)$ & Olivine-diabase ...... & Cape Colony, Africa... & N. J., 1887, V, 249.-W. T. \\
\hline 52.22 & $.048(51.9-.051)$ & $\begin{array}{l}\text { Olivine-disbase-por- } \\
\text { phyrite. }\end{array}$ & $\ldots$ do,$\ldots . . . . . . . . . .$. & Do. \\
\hline
\end{tabular}

RANG 4 .

SURPANG 3

(IIT. 4, 4, 3.)

\begin{tabular}{|c|c|c|c|c|}
\hline 56.18 & 0.036 & Diorite. & Wrshington, D. C.... & Bull. 168, 44.-W. T. \\
\hline 45.75 & .027 & Melaphyre & Holmestrand, Norway & Z. K., XVI, 27.-W. T. \\
\hline
\end{tabular}

RANG 5. -

(III. 4,5.)

\begin{tabular}{l|l|l|l|l|l}
\hline $\begin{array}{l}\text { olivine-gabbro-dia- } \\
\text { base. }\end{array}$ & Sölvberget, Norway ................ Z. K., XVI, 27.-W. T. \\
\hline
\end{tabular}


Classified list of analyses used in constructing diagrams-Continued.

Order 5. Gathare.

Rang 1. Onendase.

SUBRANG 1. ORENDOSE.

(III. 5, 1, 1.)

\begin{tabular}{|c|c|c|c|c|}
\hline $\begin{array}{c}\text { Silica } \\
\text { cont- } \\
\text { tent. }\end{array}$ & $\begin{array}{c}\text { Alkali-silica } \\
\text { ratio. }\end{array}$ & Author's name. & Locality. & Reference. \\
\hline 54.17 & 0.160 & Orendite........ & Leucite Hille, Wyo .................. & Bull. 168, 86.-W. T. \\
\hline 54.08 & .168 & $\ldots$. do $\ldots . . . . . . . .$. & $\ldots \ldots$ do $\ldots \ldots \ldots \ldots \ldots \ldots \ldots \ldots \ldots \ldots \ldots \ldots$ & Do. \\
\hline 58.70 & $.160(58.6)$ & Wyomingite... & .....do..... & Bull. 168, 85.-W. T. \\
\hline
\end{tabular}

SUBRANG 2. -

(III. 5, 1, 2.)

\begin{tabular}{l|l|l|l|l|}
\hline 50.23 & 0.150 & Wyomingite.......... & Leucite Hills, Wyo ................. Bull, 168, 85. - W. T. \\
\hline
\end{tabular}

Rang 2. Kilauase.

Subrang 2. Prowersose.

(III. $5,2,2$.)

\begin{tabular}{l|l|l|l}
\hline 50.41 & 0.113 & Syenitic lamprophyre & Two Buttes, Colo.................. Bull. 168, 165.-W. T. \\
\hline Subra NG 3. LAMarose.
\end{tabular}

(III. 5, 2, 3.)

\begin{tabular}{|c|c|c|}
\hline 48.98 & 0.107 & Shonkinite \\
\hline 48.95 & .097 & Absarokite.... \\
\hline 47. 56 & .099 & Mica-picrophyre...... \\
\hline 47.32 & $.090(47.5)$ & Leucite-gbsarokite ... \\
\hline
\end{tabular}

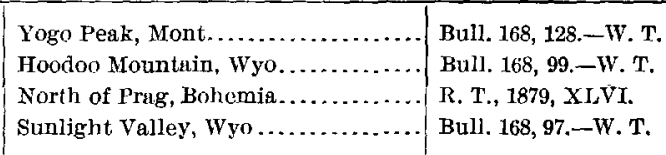

SUBRANG 4. KHLAUOSE.

(III. 5, 2, 4.)

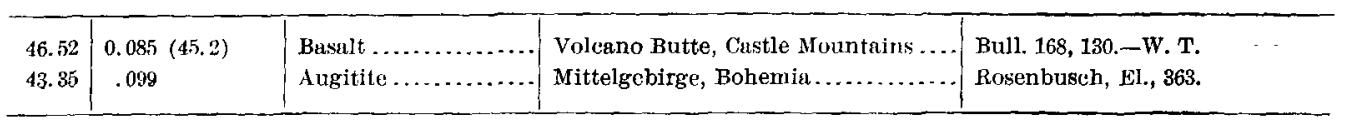

Rang 3. Campronase.

SUBRANG 2. ARSAROKOSE.

(III. 5, 3, 2.)

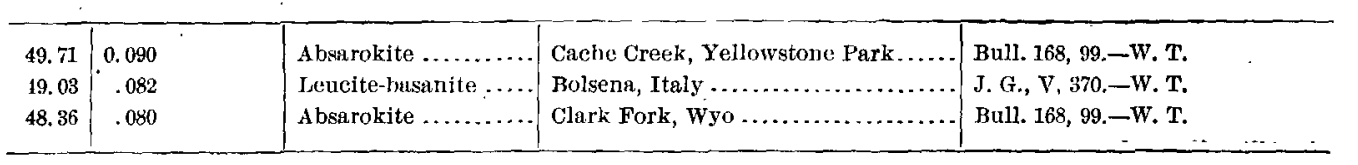


ROCK AIVALYSES USED IN CONSTRUCTING DIAGRAMS.

Classified list of analyses used in constructing diagrams-Continued,

Subrang 3. Knentallexose,

(III. $5,3,3$.

\begin{tabular}{|c|c|c|c|c|}
\hline $\begin{array}{l}\text { Silica } \\
\text { con- } \\
\text { tent. }\end{array}$ & $\begin{array}{l}\text { Alkali-silien } \\
\text { ratio. }\end{array}$ & Author's name. & Loculity. & keference. \\
\hline 52.09 & 0.074 & Kentallenite ... & Glen Shira, Argyllshire, Scothand .... & Q.J. G.S., LVI, 537.-W. T. \\
\hline 01. 76 & .083 & Absarokite & Absaroku Range, Fellowstone Park. . & Bull. 1t8, 99.-W. T. \\
\hline 51.65 & .107 & Lamprophyre. & Cottonwood Canyon, Mont.......... & Bull. 168, 112.-W. T. \\
\hline 50.82 & .075 & $\ldots \ldots$ do $\ldots . . .$. & $\begin{array}{l}\text { Antelope and South Boulder creeks, } \\
\text { Mont. }\end{array}$ & Bull. 168, 113.-W. T. \\
\hline 50.35 & .101 & Olivine-nonzonite ... & Smälingen, Sweden . . . . . . . . . . . . & E. K., II, 46,-W. T. \\
\hline
\end{tabular}

SUBRANG 4. CAMPTONOSF.

(III. 5, 3, 4.)

\begin{tabular}{|c|c|c|c|c|c|}
\hline 54,56 & 0.067 & Quartz-basalt. & Cinder Cone, Cal.. & Bull. 168, 185.-W. T. & \\
\hline 53.56 & .077 & Mica-porphyry .. & Crandall Basin, wyo.......... & Bull. $168,92-$ W. T. & \\
\hline 52. 35 & .007 & Diorite... & Little Falls, Minn.. & N. J., 1877, 129. & \\
\hline 51.81 & .077 & Gubbro-porphyry. & Crandall Basin, Wyo... & Bull. 168, 9-2.-W. T. & \\
\hline 51.23 & .076 & Diabuse. & Halleherg, Sweden.. & Rosenbusch, El., 3æs. & \\
\hline 49.85 & .074 & Dolerite......... & Puntellaria .............. & T. M. P. M., 1883, 393. & \\
\hline 48.60 & $.095(48.4)$ & Nephelite-basalt. & 'Elkhend Mountains, Colo ... & Fortietlı Par., 'T, 676 & \\
\hline 48.22 & .084 & Crimptonite... & Mount Ascutney, Vt....... & Bull, 168, 26.--W. T. & v \\
\hline 47.91 & .086 & Basult ........ & Washoe, Nev .............. & & \\
\hline 47.90 & .094 & Essexite ....... & Tofteholmen, Norway... & E. K., II, 375.-W. T. & \\
\hline 47.28 & .083 & Leucite-absarokite . & Ishawooa Cunyon, wyo........... & Bull. 168, 99.-W. T. & \\
\hline 47.16 & .077 & Gabbro: & Elizabethtown, N. Y...... & Bull. $168,37 .-\mathrm{W} . \mathrm{T}$. & \\
\hline 46.74 & .089 & .....do... & $\ldots$. do $\ldots . . . \ldots \ldots \ldots$. & Do. & \\
\hline 45.30 & .107 & Basalt .. & Mount Trumbull, Ariz .. & Bull. 168, 174.-W. T. & \\
\hline 40.60 & .078 & Cumptonite....... & Maena, Gran, Norway............. & E. K., III, 376.-W. T. & \\
\hline
\end{tabular}

SUBRANG 5. ORNOSE.

(III. 5, 3, 5.)

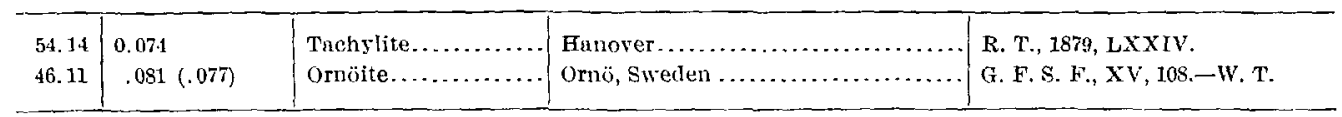

Rang 4, AUVERgNase.

SUbRaNg 2. -

(III. $5,4,2$ )

\begin{tabular}{|c|c|c|c|c|}
\hline $\begin{array}{l}50.03 \\
46.86\end{array}$ & $\begin{array}{l}0.062(49.4) \\
.065\end{array}$ & $\begin{array}{l}\text { Lamprophyre ........ } \\
\text { Diabuse-pitchstone... }\end{array}$ & 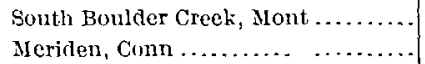 & $\begin{array}{l}\text { Bull. 168, 113.-W. T. } \\
\text { Bull, 168, 35.-W. T. }\end{array}$ \\
\hline
\end{tabular}

SURRANG 3. AUVERgNose

(III, 5, 4, 3.)

\begin{tabular}{|c|c|c|c|c|}
\hline 53.13 & 0.053 & viabase... & Jersey City, N. J. . . . . & A. J. S., IX, 1875, 187. \\
\hline 52.42 & .016 & ..... do ........... & Wintergreen Lake, Conn............. & A. J. S., IX, $1975,189$. \\
\hline 52.68 & .057 & Dolerite... & Mount Holyoke, Mass......... & A. J. S., IX, 1875, 186. \\
\hline 51.78 & .044 & Diabuse $. . . \ldots .$. & 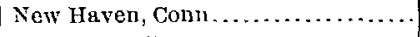 & Do. \\
\hline 51.46 & .057 & Gabbro.......... & Sturgeon Falls, Mieh.............. & Bull. 168, 70.-W. T. \\
\hline
\end{tabular}


Classified list of analyses used in consiructing diagrems-Continued.

SUbrang 3. Attverganose-Continued.

\begin{tabular}{|c|c|c|c|c|}
\hline $\begin{array}{l}\text { Silica } \\
\text { con- } \\
\text { tent. }\end{array}$ & $\begin{array}{l}\text { Alkali-silica } \\
\text { ratio. }\end{array}$ & Author's name. & Locality. & Reference. \\
\hline 51.36 & 0.040 & Basalt. & Orange, N. J... & Bull. $168,39 .-W$. T. \\
\hline 50.89 & .053 & .....do... & Lassen Peak, Cal.. & Bull. 168, 186.-W. T. \\
\hline 49.87 & .062 & .... do ......... & Pantellarin ............. & T. M. P. M1., 1883, 39\%. \\
\hline 49.63 & $.05 i(49.5)$ & Diubase .... & Mount $A$ sentney, Vt..... & Bull. $168,2 t .-W . T$. \\
\hline 49.18 & .035 & Basait .. & Disko, Greenlund. & T. M. P. M., 1874, 120. \\
\hline 48.23 & .031 & Norite ..... & Crystal Fulls, Mich. & Bull. $168,67 .-\mathrm{W} . \mathrm{T}$. \\
\hline 48.04 & .045 & Basilt ...... & Ovifak, Greenland .... & T. M. P. M., 1874, $1: 21$. \\
\hline 47.93 & $.017(4 \pi .7)$ & ....do ...... & I'nynes Creek, Cal......... & Buil. $168,186 .-W . T$. \\
\hline 47.90 & $.043(17.4-.0 .10)$ & Diabase & Penoket-Gogeljie: Range, Mich.. & Bull. 165, 73.-W. T. \\
\hline 47.54 & $.070(47.8-.079)$ & Basalt ... & Grant, N. Mex $\ldots \ldots \ldots \ldots \ldots$ & Bull. 168, 170.-W. T. \\
\hline 47.45 & .082 & Gabbro..... & Suleix, Iyrunees ..... & C. G. I., VIII, 1901, 832.-W. T. \\
\hline 47.29 & .029 & Pyroxene-ariegite & Lherz, Pyreneew .............. & C. G. I., VILI, 1901, 833.-W. T. \\
\hline .15 .66 & .049 & Olivine-gabbro & Birch Lake, Minn .: & Bull, 168, 81.-W. T. \\
\hline 44.90 & .033 & Pyroxene-iriegite. & Tue d'Ess, Pyrenees... & C. G. I., VIII, 1901, 833.-W. T'. \\
\hline 44.97 & .072 & Gabbro... & Elizabethtown, N. Y & Bull. $168,37:-$ W. T. \\
\hline 44.77 & .057 & Basalt .... & Kosk Creek, Cal..... & Bull. 168, 186.-W. T. \\
\hline 48.50 & .062 & $\begin{array}{l}\text { Hormblende-monchi- } \\
\text { quite. }\end{array}$ & Magnet Cove, Ark........ & Rusenbusch, El., 235.-W. T. \\
\hline 42.77 & .063 & Olivine-diabase & Campton, N. H .. & A. J.S. XVII, 1879, 150. \\
\hline 42.68 & .015 & Ariegite.... & Escourgeat, Iyrches............... & C. G. I., VIII, 1901, 883.-W. T. \\
\hline 42.03 & $.053(.055)$ & Jourchite .... & 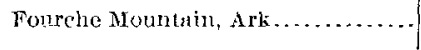 & I. R. A., 108.-W. T. \\
\hline
\end{tabular}

Rang 5. Kedabekase.

(III. 5, 5.)

\begin{tabular}{|c|c|c|c|c|}
\hline 18. 02 & 0.010 & Norite.. & McKinseys Mill, Md......... & Bull. $168,45 .-\mathrm{W} . \mathrm{T}$. \\
\hline 47.09 & .014 & Ariegite ....... & Lherz, Pyrenees.... & C. G. I., VIII. 1901. 83\%,-W. T. \\
\hline 44.76 & .020 & Hrpersthene-gabbro. & Wethererlvilla, Md. & BuII. 168, 44.-W. T. \\
\hline 44.64 & $.017(45.1)$ & Kedabelite. & Kedabek, Kussilı... & W. T. \\
\hline 44.38 & $.019(43.8)$ & Ariegite .... & Lherz, Pyrences... & c. G. I., VII, 1901, s;3.-W. T. \\
\hline 44.11 & .012 & Kedabekite.. & Kedabez, Russiat... & W. T. \\
\hline 42.32 & .025 & Ariegite .... & Lherz, Pyrenees..... & C. G. 1., V1II, 1901, s83.-W.T. \\
\hline 38.95 & .029 & .....do. & .....do .. & Do. \\
\hline
\end{tabular}

Order 6. Portugare.

RANG 1. WYOMINGASE.

Subrang 3.

(IIJ. 6, 1, 3.)

\begin{tabular}{l|l|l|l|l|l}
\hline 50.00 & 0.110 & Shonkinite .......... & Betirpaw Nountains, Mont.......... \\
\hline
\end{tabular}


ROCK ANALYSES USED IN. CONSTRUCTING DIAGRAMS.

Classified list of analyses used in constructing diagrams-Continued.

Rant 2. Monchiquase.

Subrang 3. ShoNkinose.

(III. 6, 2, 3.)

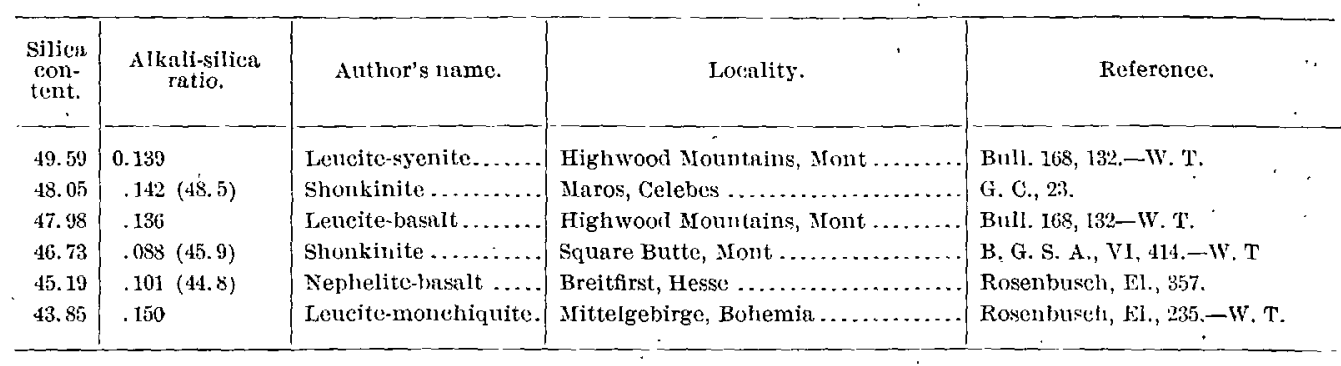

SubraNg 4, MoNchiquose.

(III. $6,2,4$. )

\begin{tabular}{|c|c|c|}
\hline 48.43 & 0.114 & Hornblende-vogesite. \\
\hline 47.82 & .121 & Monchiquite ... \\
\hline 45.59 & $.110(45.8)$ & Aralcite-basalt. \\
\hline 45.56 & .154 & Nephelite-tephrite ... \\
\hline 44.54 & .124 & Limburgite. ....... \\
\hline 43.84 & .119 & Leucite-basunite ... \\
\hline
\end{tabular}

Hohwald, Elsass ................. Rosenbuseh, El, 23I.

Highwool Mountains, Mont ......... Bull. 168, 132.-W. T.

Pikes Peak district, Colo ............. Bull, 168, 146.-W. T.

Herrnberg, Tetschen, Bohemia ...... W. T.

Heldburger Feste, S. Thïringen ..... Rosenbusch, El., 363.

Blankenhornberg, Kaiserstuhl, Baden W. T.

RaNG 3. Limhurgase.

Surrang 3. Ourose.

(III. 6, 3, 3.)

\begin{tabular}{|c|c|c|}
\hline 46.65 & 0.114 & Labrudorite-porphyt \\
\hline 43.74 & .108 & Monehiquite. \\
\hline 12. 46 & .068 & . do \\
\hline
\end{tabular}

Wildkuar, Tyrol ................. R. T., 1879, XLIV.

Rio do Ouro, Bruzil.................. Rusenbuseh, El., 235.-W. T.

Willow Creek, Mont................ Bull. 168, 130.-W. T.

SUBRANG 4. LIMBURgose.

(IJT, 6, 3, 4.)

\begin{tabular}{|c|c|c|}
\hline 46.60 & $0.112(46.3)$ & Nephelite-gubbro \\
\hline 46.35 & .086 & Neplielite-basunite. \\
\hline 44.82 & $.073(44.5-.074)$ & Paleolimburgite.. \\
\hline 43.65 & $.071(43.8)$ & $\begin{array}{l}\text { Olivint-gabbro-d ia - } \\
\text { bnse. }\end{array}$ \\
\hline 43.50 & .107 & Augitite.......... \\
\hline 41.01 & $.08 \%$ & Hornblende-basalt \\
\hline 40.70 & $.084(40.5)$ & Limburgite .... \\
\hline 40.10 & $.109(40.4)$ & Ijolite. \\
\hline 37.90 & $.09 \overline{5}$ & Horublendite. \\
\hline
\end{tabular}

Nosy Komba, Madagnscar.......... M. M., 204.

Römhild, S. Thüringen .............. Rosenbusch, El., 346.

Atachma, Chile ................... $z$. D. g. G., LI, 4.

Brandberget, Gran, Norway ......... Q. J. G. S., L, 19.-W. 'T.

Limberg, Buden................... w. r

Sparbrod, Rhone.................. N. J., 1882, II, 155.

Oberersteinberg, Lausitzer Moun- Rosenbusch, EI. 363. tains, l'russia.

Ampasibitika, Madagascar........... M. M., 204.

Brandberget, Gran, Norwas.......... Q. J. G. S., L, 19.-W. T.

Subrang 5. -

(III. $6,3,5$.)

\begin{tabular}{r|c|c|c|c|}
\hline 41.94 & 0.121 & Camptonite ......... & Campton Falls, N. H ............... A. J. S., XVII, $1879,150$. \\
\hline
\end{tabular}


Classified list of analyses used in constructing diagrams-Continued.

RANG 4.

Subrang 1. -

(III. $6,4,1$.)

\begin{tabular}{l|c|c|c|c}
\hline $\begin{array}{c}\text { Silica } \\
\text { con. } \\
\text { tent. }\end{array}$ & $\begin{array}{c}\text { Alkali-silica } \\
\text { ratio. }\end{array}$ \\
\hline 39.84 & $0.046(40.4)$ & Author's name. & Locality. & Refercunce. \\
\hline
\end{tabular}

SUURANG 3.

(IiI. 6, 4, 3.)

\begin{tabular}{l|l|l|l|l|}
\hline 45.40 & 0.063 & Dioritic gabbro...... & Nosy Komba, Madagascar ........... M. M., 204. \\
\hline
\end{tabular}

Order 7. Kamerunare.

RANG 1, MAIGNASE.

SUBRaNG $3 .-$

(III. $7,1,3$. )

\begin{tabular}{l|l|l|l|l|}
47.85 & 0.144 & Nephelite-malignite. & Poohbah Iake, Ontario .............. & Rosenbuseh, EI., 176.-W. T. \\
\hline
\end{tabular}

SUbrang 4. MaLignose.

(II, 7, 1, 4.)

\begin{tabular}{|c|c|c|c|c|}
\hline 51.88 & 0.180 & $\begin{array}{l}\text { Garnet-pyroxene- } \\
\text { malignite. }\end{array}$ & Poohbuh Lake, Ontario. & Rosenbusch, El., 176.-W. T. \\
\hline 51.38 & .193 & Amphibole-malignite & ..... do. & Do. \\
\hline 44.65 & .186 & Theralite............ & Crazy Mountains, Mont .............. & Bull. 168, 124.-W. T. \\
\hline 42.02 & $.161(41.8)$ & Ijolite-porphyry..... & Alnö, Sweden ................... & N. J., 1897, II, 99.-W. T. \\
\hline
\end{tabular}

Rang 2. KaMerunase.

SUBRANG 3. - -

(1II. 7, 2, 3.)

\begin{tabular}{|c|c|c|c|c|}
\hline 46.04 & 0.127 & Lcucite-basalt. & Highwood Mountains, Mont ......... & Buil. 168, 132.--W. T. \\
\hline
\end{tabular}

SUbrang 4. Kamerunose.

(III, 7, 2, 4.)

\begin{tabular}{|c|c|c|c|c|}
\hline 47. 10 & 0.178 & Heumite.. & Laugendal, Norway .. & E. K., JII, 376.-W. T. \\
\hline 46.53 & .123 & Therulite. & Umptck, Finland. & Rosenbusch, El., 170.-W. T. \\
\hline 45.77 & .163 & Furrisite... & Farris, Norway....... & F. K., III, 376.-W. T. \\
\hline 44.31 & .147 & Theraite. & Gordons Butte, Mont.. & Bull. 168, 124.-W. T. \\
\hline 42.77 & $.143(43.0)$ & Monchiquite... & Kiechlinsbergen, Baden........ & W. $\mathrm{T}$. \\
\hline 42.65 & $.133(43.3)$ & Basalt .......... & Cape Verde, Africa............. & R. T., 1884, LXXIV. \\
\hline 40.10 & $.167(40.3)$ & Icucitenephelinite. & Etinde Volcano, Samerun, Africa.... & W. T. \\
\hline 39.97 & $.183(40.2)$ & $\ldots \ldots$ do $\ldots . . . . . . . .$. & $\ldots$ do $\ldots \ldots \ldots \ldots \ldots \ldots \ldots \ldots$. & Do. \\
\hline 39.30 & $.165(40.8)$ & Haüynophyre & ... do .... & Do. \\
\hline
\end{tabular}


Classifled list of analyses used in constructing diagrams-Continued.

Rang 3. Etindase.

SÜBRANG 4. ETINDOSE.

(III, 7, 3, 4.)

\begin{tabular}{|c|c|c|c|c|}
\hline $\begin{array}{l}\text { Silica } \\
\text { con- } \\
\text { tent. }\end{array}$ & $\begin{array}{l}\text { Alkali-silica } \\
\text { ratio. }\end{array}$ & Author's name. & Locality. & Reference. \\
\hline 40.52 & 0.097 & Nephelite-basalt.. & Hesse-Darmstadt. & Rosenbusch, El., 357. \\
\hline 39.33 & .109 & ..... do ...... & Mittelgebirge, Rohemia...... & Do. \\
\hline 38.46 & $.143(38.9)$ & Camptonite & Mulatto, Predazzo, Tyrol............. & K. A. W. W., CXI, I, 234. \\
\hline
\end{tabular}

Order 8. Bohemare.

Rang 1. Chotask.

SUBRANG 2. ChOTOSE.

(III. 8, 1, 2.)

\begin{tabular}{l|l|l|l|l|}
\hline 46.51 & 0.167 & Leneitite ............ Bearpaw Mountains, Mont.......... Bull. 168, 136...W.T. \\
\hline
\end{tabular}

Rang 2. Albanase.

Subrang 2. Albanoge.

(III. 8, 2, 2.)

\begin{tabular}{l|c|l}
47.47 & 0.137 \\
45.99 & $.168(46.1)$ & $\begin{array}{l}\text { Leucite-tephrite...... } \\
\text { Leucitite } \ldots \ldots \ldots . . .\end{array}$ \\
\hline
\end{tabular}

Vesuvius, lava of 1760

R. T., 1884, LVI.

Capo di Bove, Italy ................ A.J.S., IX, 1900, 53.-W.T.

Subrang 3 . -

(III. $8,2,3$. )

\begin{tabular}{|c|c|c|}
\hline 41.24 & 0.141 & Nephelinite ... \\
\hline
\end{tabular}

\begin{tabular}{l|l|l} 
Laacher See, Rhine province ........ & Rosenbusch, El., 357.
\end{tabular}

Subrakg 4. Covose.

(III. $8,2,4$ )

\begin{tabular}{|c|c|c|c|c|}
\hline 43.18 & 0.186 & Theralite.. & Crazy Mountains, Mont ...... & Rosenbusch, El., 176. \\
\hline 42,06 & .132 & Timpurgite........... & Habichtswald, Hesse................. & Rosenbusch, Nl., 363. \\
\hline 41.74 & .204 & Ijolite ............. & 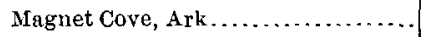 & J.G., IX, 618.-W.T. \\
\hline 40.15 & $.204(40.3)$ & Nephelinite: & Etinde Volcano, Kamerun, Africa ... & W. T. \\
\hline 38.39 & .150 & ....do.... & 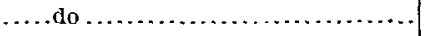 & Do. \\
\hline
\end{tabular}

\section{Order 9. Finnare.}

Rang 1. IJotase.

Subrang 2. Madupose.

(III. 9, 3., 2.)

$42.65 \mid 0.139 \quad$ Madupite........... $\mid$ Leneite Hills, Wyo ...............


Classified hist of analyses used in constructing diagrams-Continued.

SUBRANG 4. IIVAAROSE.

(III. 9, 1, 4.)

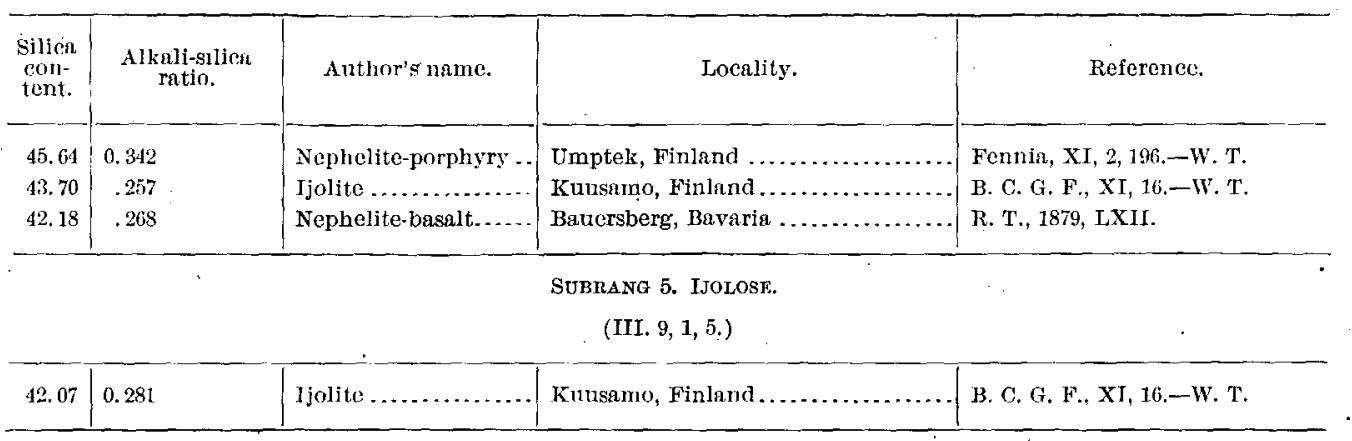

CLASS IV. DOFEMANE.

Order 1. Hungarare.

SECTION 1. MINNESOTIARE.

Rang 1. Minnesotase.

SECTION 1. MINNESOTIASE.

Subrang 2. Cookose.

(IV. 1, 1, 1, I, 2.)

\begin{tabular}{|c|c|c|c|c|}
\hline 51.83 & 0.005 & Jyroxenite. & Meadow Creek, Mont... & Bull. 168, 114.-W. T. \\
\hline 50.64 & .020 & Wehrlite ........... & New Braintree, Mass..... & Bull. 168, 83,-W. T. \\
\hline 46.90 & .030 & Hypersthene-sabbro. & Gunflint Take, Minn...... & Bull. 168, 81.-W.T. \\
\hline
\end{tabular}

SECTION 2. - .

RANG 1.

SECTION 2.

Subrakg 1. Belcherose.

(IV. 1, 2, 1, 2, 1.)

\begin{tabular}{|c|c|c|c|c|}
\hline 48.63 & 0.008 & Peridotite & Belehertown, Mass............ & Bull. 168, 30.-W. T. \\
\hline$\therefore$ & & r. & $\begin{array}{l}\text { SUbra } \\
\text { (IV. } 1,2,1,2,2 \text { ) }\end{array}$ & · \\
\hline 48.91 & 0.013 & Olivine-gabbro ....... & Orange Grove, $\mathrm{Md} . \ldots \ldots \ldots \ldots \ldots$ & Bull, 168, 44.-W. T. \\
\hline 46.56 & .050 & Gabbro ..... & Pharkowsky-Ouwal, Ural Mountains. & O. N., 145. \\
\hline 46.56 & $.043(40,9)$ & .....do.... & Near Koswinsky, Ural Mountains.... & Do. \\
\hline 46.06 & .098 & Missourite... & Shonkin Creek, Mont.............. & Bull. 168, 139.-W. T. \\
\hline 44.94 & .000 & Wohrlite...$\ldots \ldots \ldots$ & Koswinsky, Ural Mountains......... & O. N., 173. \\
\hline 44.39 & .032 & Gabbro $\ldots . . . . . .$. & Pharkowsky-Ouwal, Ural Mountains. & O. N., 145. \\
\hline
\end{tabular}


ROGK ANALYSES USED IN CONSTRUCTING DIAGRAMS.

Clussified list of unalyses used in constructing diugrums-Continted.

SECTION 3. HUNGARIARE.

RANG 1. WhHRLASE.

SECTION 1. WEHRLIASE.

SUbrang 2. We,hriose.

(IV. $1,3,1,1,2$.)

\begin{tabular}{|c|c|c|c|c|}
\hline $\begin{array}{l}\text { Silica } \\
\text { con- } \\
\text { tent. }\end{array}$ & $\begin{array}{c}\text { Alkali-silica } \\
\text { ratio. }\end{array}$ & Author's name. & Locality. & Reference. \\
\hline 46.95 & 0.040 & Wehrlite & Red Bluff, Mont............ & Bul1, 168, 114.-W. T. \\
\hline 46.13 & .001 & Iornblende-picrite. & Meadow Creek, Mont.......... & Do. \\
\hline 45. 68 & .000 & Lherzolite.......... & Baldisser, Piedmont, Italy . ....... & Rosenbusch, El, 165. \\
\hline
\end{tabular}

SECTION 2

SUbIANg 2. Rosswbinose.

(TV. 1, 3, 1, 2, 2.)

\begin{tabular}{|c|c|c|c|c|}
\hline 47.75 & 0.027 & Peridotite. & Cathay Hill, Mariposa County, Cal... & Bull. 168, 209.-W. T. \\
\hline 46.00 & $.085(46.1)$ & Homblendite. & Col d'Eret, Pyrenees ................ & C. G. I., VIIT, 1901, 833.-W. T. \\
\hline 44.99 & .028 & Wehrlite ...... & Crystal Falls, Mich...... & Bull. $168,67$. W. T. \\
\hline
\end{tabular}

\section{SECTION 4. -}

RANG 1. CohtuanDTASE.

SECTION 1, CORTLANDTIASE.

Subrang 1. CoRtrandtosk.

(IV. $1,4,1,1,1$ )

\begin{tabular}{|c|c|c|c|c|}
\hline 44.61 & 0.000 & Lherzolite. & Lherz, l'yronees.. & C. G. I., VIII, 1901,833 - \\
\hline 36.80 & .049 & Peridotite ........... & Syracuse, $N \mathrm{Y} \ldots \ldots \ldots \ldots \ldots$ & J311, $168,38 .-W . T$. \\
\hline
\end{tabular}

Subrang: 2. Custerose.

(IV: $1,4,1,1,2$.)

\begin{tabular}{l|l|l|l|l|}
\hline 46.03 & 0.041 & Peridotite ........... & Silver Cliff, colo.................. & Buit. 168, 147.-W. T. \\
\hline
\end{tabular}

SECTION 5. PYRENIARE.

Rang 1. Literzase.

SECTION 1. LHERZIASE.

SUBRANG 1. LUERZOSE.

(IV. 1, 5, 1, 1, 1.)

\begin{tabular}{|c|c|c|c|c|}
\hline 41.50 & 0.036 & Hornblende-1herzolite & 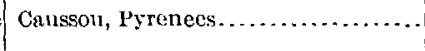 & C. G. T., 1901, VITI, \$33,-W. T. \\
\hline
\end{tabular}

\begin{tabular}{l|l|l|l|l|l}
39.25 & 0.085. & $\begin{array}{l}\text { Hornblende-perido- } \\
\text { tite. }\end{array}$ & Argein, Pyrenees.................. G. G. I., 1901, VIII, 838., W. T. \\
\hline
\end{tabular}


Classified list of analyses used in constructing diagrams-Continned.

SEGTION 3. VENANZIASE.

SUPRANG 2. VENANZOSE.

(IV. I, 5, 1, 3, 2.)

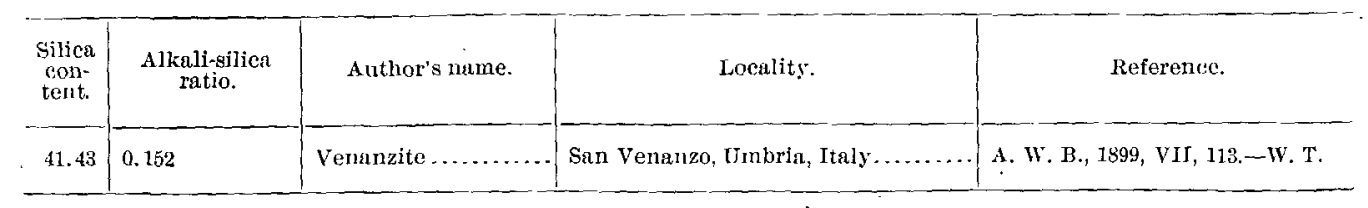

\section{Order 2. Scotare.}

\section{SECTION 1 .}

Rang 1.

SECTION 1.

Sumtaxa 2.

(IV. 2, 1, 1, 1, 2.)

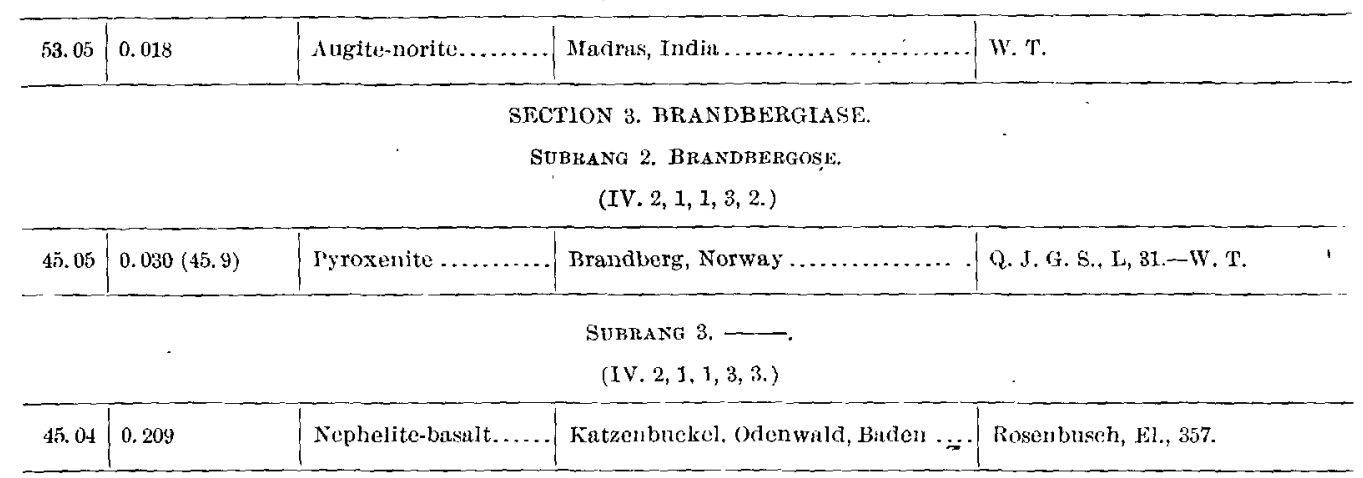

SECTION 2. PAOLIARE.

Rang 1. Paolase.

SECTION 2. -

SUBRANG 2. -

(IV. 2, 2, 1, 2, 2.)

\begin{tabular}{|c|c|c|c|c|}
\hline 47.41 & 0.031 & Augite-peridotite. & Ireekskill, N. Y. & A. J. S., $\times \times \times 1,1 \operatorname{sis} 6,40 .-1 \%$. \\
\hline 42.68 & .077 & Hornblende-basalt. & Hauk, S. Rhone....... & Rosenbusch, El., 309. \\
\hline 40.42 & .038 & Olivine-gabbro... & Crazy Mountains, Mont ........ & Bull. 168, 122.-W. T. \\
\hline 40.15 & .000 & Kowwite........... & Koswinsky, Urul Mountains .......... & O. N., 119 \\
\hline 39.59 & .057 & Nepheilte-basalt ... & 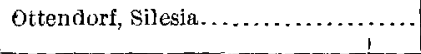 & R. 'T., 1884, LXIV. \\
\hline
\end{tabular}

SECTION 3. PAOLIASE.

Subrang 2. Paolose.

(IV. $2,2,1,3,2$.

\begin{tabular}{|c|c|c|c|c|}
\hline 41.44 & $0.000(41.6)$ & Koswite.... & Koswinsky, Ural Mountains.......... & O. N., 119. \\
\hline 38.39 & $.031(37.5)$ & Jacupirangite & Magnet Cove, Ark... & J. G., IX, 620,-W. T. \\
\hline 38.38 & .022 & ..... do ........ & Silo Paulo, Brazil... & Do. \\
\hline 36.51 & .075 & Eleolite-mica-syenite. & Magnet Coye, Ark ................... & Do. \\
\hline
\end{tabular}


Classified list of analyses used in constructing diagrams-Continued.

SECTION 3. TEXIARE.

Rang 1. Texase.

SECTION 1. MARQUETTIASE.

SUBRANG 2. MARQUETTOSE.

(IV. $2,3,1,1,2$.

\begin{tabular}{c}
$\begin{array}{c}\text { Silica } \\
\text { con- } \\
\text { tent. }\end{array}$ \\
\hline 39.87
\end{tabular} \begin{tabular}{c}
$\begin{array}{c}\text { Alknil-siliea } \\
\text { rutio. }\end{array}$ \\
\hline 0.017
\end{tabular}

SEC'TION 2. UVALDLASE.

Stubravg 2. UVhtidose.

(IV. 2, 3, 1, 2, 2.)

\begin{tabular}{|c|c|c|c|c|}
\hline 40.32 & 0.080 & Nephelite-basalt & Uvalde County, Tex. & Bull. 168, 62.-W. T. \\
\hline 39.92 & $.063(11.0)$ & ..... do... & $\ldots \ldots$ do $\ldots \ldots \ldots \ldots . . .$. & Bull. 168, 63.-W. T. \\
\hline 39. 16 & .081 & ..... do do........... & Oberleinleiter, Buden. & losenbusch, El., 357. \\
\hline 36.53 & .108 & $\begin{array}{l}\text { Nephulite-molilite- } \\
\text { basalt. }\end{array}$ & Wartenberg, Baden ................ & Rosenbuseh, EI., 360 . \\
\hline
\end{tabular}

SFCTION 4

Rang 1. Casselase.

SECTION 2. CASSFLIASE.

Subrang

(IV. 2, 4, 1, 2, 1,)

\begin{tabular}{|c|c|c|c|c|}
\hline $\begin{array}{l}38.34 \\
37.98\end{array}$ & $\begin{array}{l}0.106 \\
.121(37.4)\end{array}$ & $\begin{array}{l}\text { Limburgite.......... } \\
\text { Melilite-nephelite- } \\
\text { basalt. }\end{array}$ & 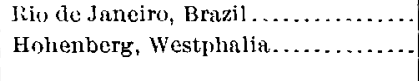 & $\begin{array}{l}\text { T. M. I. M., XX, } 304 . \\
\text { Rosenbuseh, El, } 357 .\end{array}$ \\
\hline
\end{tabular}

Subrang 2. Cassejose.

(IV. $2,4,1,2,2$ )

\begin{tabular}{|c|c|c|c|c|}
\hline 39.20 & $0.056(38.6)$ & Dike-rock ........... & Kola Peninsula, Finland.. & Fennia, 15, 2, 1899, 27.-W.T. \\
\hline 37.96 & .066 & $\begin{array}{l}\text { Nephelite-nelilite- } \\
\text { busult. }\end{array}$ & Uvalde County, Tex ... & Bull. 168. 63.-W. T. \\
\hline
\end{tabular}

SFCTION 5

RANG 1.

SECTION 1. KALTENIASE.

Subrang 3. Kaltenosh.

(IV. 2, 5, 1, 1, 3.)

\begin{tabular}{l|l|l|l|l}
\hline 34.98 & 0.101 & Mica-peridotite....... & Kaltenthal, Harzburg, Harz Mts ...... Rosenbuseh, El., 165.--W. T. \\
\hline
\end{tabular}


Chassified list of amolyses used in. construtting diagroms-Continned.

Order 3. Sverigare.

SECTION 1. BERGENIARE.

Rang 1. Beigenase.

SECTION 1. BERGENTASE.

SUDRANG 3. BERGENOSE.

(IV. $3,1,1,1,3$.)

\begin{tabular}{|c|c|c|c|c|}
\hline $\begin{array}{l}\text { Silica } \\
\text { con- } \\
\text { tent. }\end{array}$ & $\begin{array}{l}\text { Alkali-siliea } \\
\text { ratio. }\end{array}$ & Anthor's name. & Locality. & Roference. \\
\hline 31.59 & 0.082 & Ilmenite-norite.. & Storgangen, Norway .. & R. M. A., 1S96, V, 165. -W. T. \\
\hline
\end{tabular}

SECTION 2.

RAN; 1 .

SECIION 2. -...

SUbrang 2 .

(TV. $3,2,1,2,2$.)

\begin{tabular}{|c|c|c|c|c|}
\hline 38.20 & 0.122 & $\begin{array}{l}\text { Nephelite-molilite- } \\
\text { busalt. }\end{array}$ & Burgstall, Jaden ................... & Rosenbusch, El., 360.--iW. T. \\
\hline 38.18 & .036 & (P) Pyroxenite...... & Predazzo, Tyrol & E. K, $1 \mathrm{I}, 70 .-\mathrm{V} . \mathrm{T}$. \\
\hline
\end{tabular}

SECTION 3. AVEZACIASE.

SUBRANG 3. AVEzacose.

(IV. $3,2,1.3,3$. )

\begin{tabular}{l|l|l|l|l}
\hline 1.80 & 0.026 & Avezacite ............ Avezac-Prat, Pyrences .............. G. G. I., VIII, 1901, $832,-W$. T. \\
\hline
\end{tabular}

Ordfr 4. Adirondackare.

Suborder 2. Adirondackore.

RANG 1. AdrondaGkash.

SECTION 1. ADIRÓNDACKIASE.

SUBRANG 3 . -
(IV. $4,2,1,1,3$.)

\begin{tabular}{|c|c|c|c|c|}
\hline 24.74 & 0.000 & Iron ore..... & Ulfö, Sweden ........... & S. M. Q., XXI, 60. \\
\hline 22.87 & .000 & Ironore.. & Cumberland Hill, R. I. & s. M. Q., XXI, 60 . \\
\hline 14.95 & .000 & ..... do .......... & Launghult, sweden ................. & Do. \\
\hline
\end{tabular}

SURRANG 4, -

(IV. 4, 2, 1, 1, 4.)

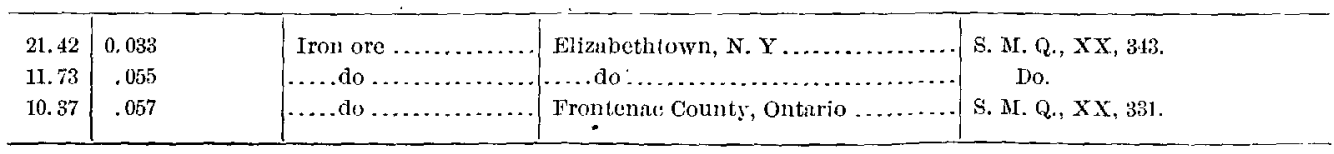


Classifierl list of analyses used in construcling diagrams-Continued.

Suborder 3. Champlainore.

Rang 1. Champlainase.

SECTION 1. CHAMPLAINIASE.

SUbrang 4.

- (IV. 4, 3, 1, 1, 4.)

\begin{tabular}{|c|c|c|c|c|}
\hline $\begin{array}{l}\text { Silica } \\
\text { eon- } \\
\text { tent. }\end{array}$ & $\begin{array}{l}\text { Alkali-siliea } \\
\text { ratio. }\end{array}$ & Anthor's name. & Locality. & Refereńce. \\
\hline 17.90 & 0.000 & Iron ore. & Split Rock mine, N. Y.. & S. M. Q., XX, 343 . \\
\hline 13.35 & .000 & ......do ... & Turnel Hill, s. Y ...... & Do. \\
\hline
\end{tabular}

Order 5.

Suborder 2,

RANG 1 .

SECTION 1

SUBRANG 4.

(IV. $5,2,1,1,4$.

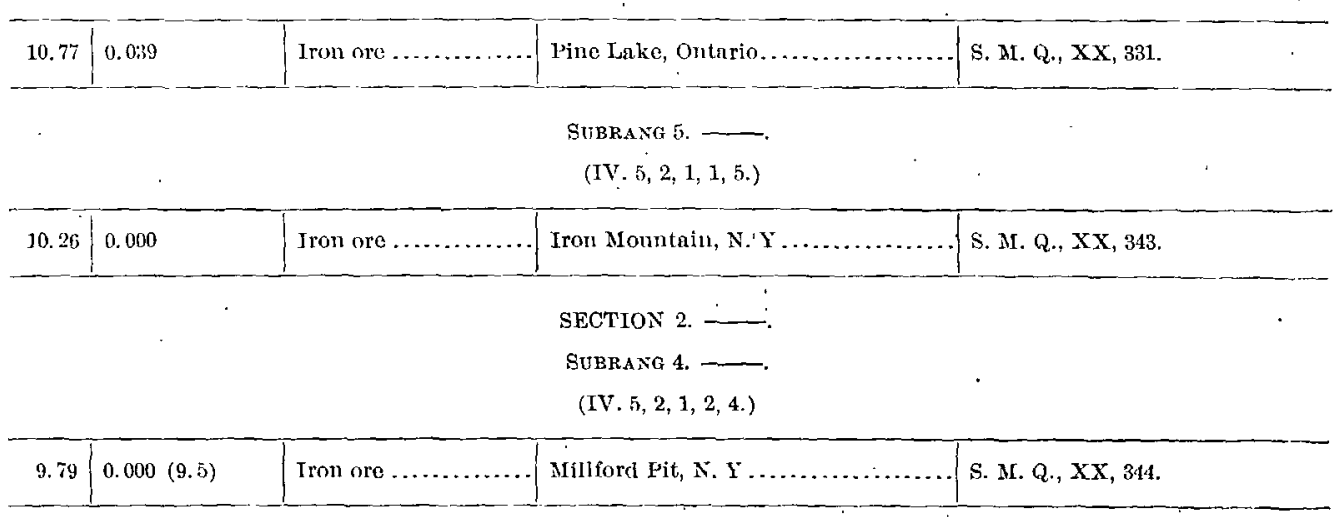

CLASS V. PERFEMANE.

Order 1. Maorart.

SECTION 1. CAROLINIARE

RaNe 1. Webs'terase.

SECTION 1. MARTCIASE.

StUBRANG 1. MLARICOSE.

(V. $1,1,1,1,1$.)

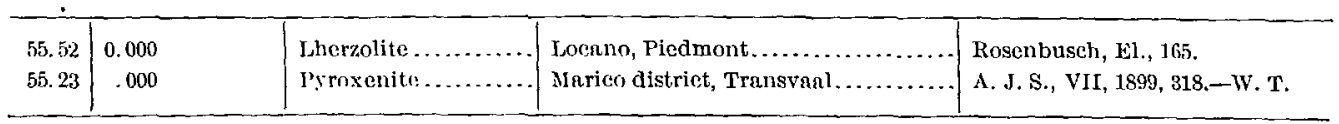


Classified lisl of analyses used in constructing diagrams-Continued. SECTION 2. WEBSTERIASE.

Subrang 1. Websterose.

(V. 1, 1, 1, 2, I.)

\begin{tabular}{|c|c|c|c|c|}
\hline $\begin{array}{c}\text { Silica } \\
\text { con- } \\
\text { tent. }\end{array}$ & $\begin{array}{c}\text { Alkali-silica } \\
\text { ratio. }\end{array}$ & Author's name. & Locality. & Reference. \\
\hline 55.14 & $0.005^{\circ}$ & Websterite... & Webster, N. C ..... & Bull. 168, 58.-W. T. \\
\hline 53.98 & .000 & ..... do ... & Hebbville, Ma ..... & Bull $168,43 .-$ W. T. \\
\hline 52.55 & .004 & ...do. & ..... do ... & Bun. 168, 43.-W. T. \\
\hline
\end{tabular}

Subrang 2. Cecilose.

(V. 1, 1, 1, 2, 2.)

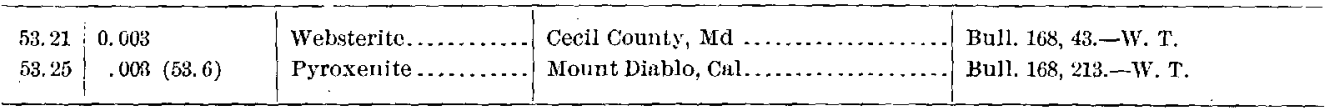

SECTION 2, MARYLANDIARF.

Rang 1. Battimorase.

SECTION 2. RALTIMORIASE.

SUBRANG 2. BALTIMorose.

(V. $1,2,1,2,2$.)

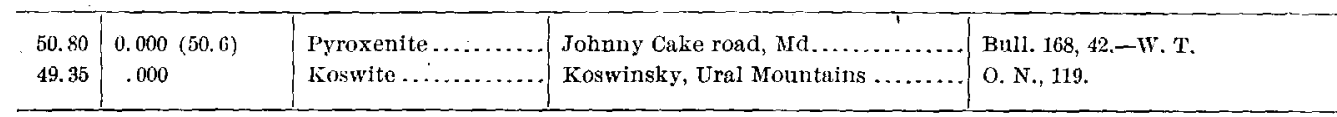

SECTION 3.

RANG 1.

SECTION 2

Subrang 2. Koswose.

(V. $1,3,1,2,2$.)

\begin{tabular}{l|l|l|l|l|}
\hline 4.20 & 0.00 & Koswite ............ & Koswingky, Ural Mountains ........ & O. N., I19. \\
\hline
\end{tabular}

SFCTION 4.

RANG 1 .

SECTION 1

Subrang 1.

(V. 1, 4, 1, 1, 1.)

\begin{tabular}{|c|c|c|c|c|}
\hline 41.43 & 0.00 & Saxonite. & Riddles, Oreg........................... & Bull. 168, 221.-W. T. \\
\hline & & \multicolumn{3}{|c|}{$\begin{array}{c}\text { Subrang } 2 . \\
(\mathrm{V} .1,4,1,1,2 .)\end{array}$} \\
\hline 42.39 & 0.000 & Peridotite & Goose Buy, Struits of Magellan. & W. T. \\
\hline
\end{tabular}


Classified list of amalyses used in constructing alagrams-Continued.

SECTION 5. MAORIARE.

Rang 1. Dunase.

SECTION 1. DUNIASE

SUbRang 1. DUnose.

(V. $1,5,1,1,1$.)

\begin{tabular}{|c|c|c|c|c|}
\hline $\begin{array}{c}\text { Silica } \\
\text { con- } \\
\text { tent. }\end{array}$ & $\begin{array}{l}\text { Alkali-silica } \\
\text { ratio. }\end{array}$ & Author's name. & Locality. & Reference... \\
\hline 42.50 & 0.000 & Dunite. & Dun Mountains, New Zealand. & Rosenbusch, rL., 165. \\
\hline 40.11 & .000 & Dunite ........... & Corundum Hills, $\mathrm{N}, \mathrm{C} \ldots \ldots \ldots \ldots \ldots$ & BuIl. 168, 54.-W. T. \\
\hline 39.99 & $.00(39.7)$ & Haraburgite...... & Olivine Hills Range, New Zealand... & Rosenbusch, E1., 165. \\
\hline
\end{tabular}

ORder 2.

SECTIONं 2.

RANG 1.

SECTION 2 .

Subrang 2.

(V. $2,2,1,2,2$ )

\begin{tabular}{l|l|l|l|l|l}
\hline 43.87 & 0.000 & Lherzolite ........... Johnny Cake road, Md............. Bull. 168, 42. \\
\hline
\end{tabular}

SECTION 5.

RANG 1.

SESTION 1 .

Surrang 2. Permose.

(V. $2,5,1,1,2$.)

\begin{tabular}{l|l|l|l|l|l|}
\hline 1.84 & 0.000 & Dunite sideronitique. & Koswinsky, Ural Mountains ......... & O. N., 128. \\
\hline
\end{tabular}

Order 3.

SECTION 4

RANG 1. - -

SECTION 1.

SUBRANG 2. -

(V. 3, 4, 1, 1, 2.)

\begin{tabular}{|c|c|c|c|c|}
\hline 21.25 & 0.000 & Iron ore $\ldots \ldots \ldots \ldots$ & Taberg, Sweden ............... & S. M. Q., XXI, 60. \\
\hline
\end{tabular}


Classified list of analyses used in constructing diagrums-Continued.

\section{Ondhe 4.}

Suborder 2.

RANG 1.

SECTION 1.

SUBRANA :.

(V. 4, 2, J, 1, 2.)

\begin{tabular}{|c|c|c|c|c|}
\hline $\begin{array}{l}\text { Silina } \\
\text { cont. } \\
\text { tent. }\end{array}$ & $\begin{array}{l}\text { Alkali-silic:a } \\
\text { rutio. }\end{array}$ & Author's name. & Locality. & Reference. \\
\hline 20.45 & 0.000 & Iron ore. & Cumberlaud Hill, R. I .... & S. M. Q., XX, 351. \\
\hline & . & & $\begin{array}{l}\text { Subrayg } 4 .- \\
\text { (V. } 4,2,1,1,4)\end{array}$ & \\
\hline $\begin{array}{r}16.17 \\
7.92\end{array}$ & $\begin{array}{r}0.000 \\
.088\end{array}$ & $\begin{array}{l}\text { Iron ore } \ldots . . . \\
\ldots \ldots \text { do } \ldots . . .\end{array}$ & $\begin{array}{l}\text { Iglamala, Sweden ........................ } \\
\text { Horton, Renfrew County, Cunadia ... }\end{array}$ & $\begin{array}{l}\text { S. Mr. Q., XXI, } 60 . \\
\text { s. M. Q., XX, } 331 .\end{array}$ \\
\hline
\end{tabular}

\section{Order 5.}

Suborder 2.

RaNar 1.

SECTION 1

SUBRANG 2

(V. 5, 2, 1. 1, 2.)

\begin{tabular}{|c|c|c|c|c|}
\hline 4.08 & 0.092 & Magnetite-spinclite .. & Routivara, Swedent........ & $Z, P, G$ \\
\hline & & \multicolumn{3}{|c|}{$\begin{array}{l}\text { SUBRANG } 3 .- \\
(\text { V. } 5,2,1,1,3 .)\end{array}$} \\
\hline $\begin{array}{l}7.52 \\
6.88\end{array}$ & $\begin{array}{r}0.080 \\
.000\end{array}$ & $\begin{array}{l}\text { Iron ore . } \\
\ldots \ldots \text { da } \ldots\end{array}$ & $\begin{array}{l}\text { Leeds County, Onturio... } \\
\text { Aluö, Sweden ............ }\end{array}$ & \multirow[t]{2}{*}{$\begin{array}{l}\text { S. M. Q., XX, } 331 \text {, } \\
\text { S. M. Q., XXI, } 60 .\end{array}$} \\
\hline & & - & $\begin{array}{l}\text { Subrang } 5 . \\
(\text { V. } 5,2,1,1,5)\end{array}$ & \\
\hline 3. 67 & 0.000 & Iron ore & Mill Pond pit, N. Y. & \multirow{4}{*}{$\begin{array}{l}\text { S. M. Q., } \mathrm{XX}, 34 . \\
\text { S. M. Q., } \mathrm{XX}, 337 . \\
\text { S. M. Q., XX, 331. } \\
\text { s. M. Q., XX, 314. }\end{array}$} \\
\hline 2.02 & .000 & .....do...... & Mayhew Iron Range, Minn .......... & \\
\hline 1,47 & $: 000$ & $\mid \ldots$ do $\ldots \ldots \ldots+\ldots, \ldots$ & $\begin{array}{l}\text { Eugle Lake'mine, Frontenac County, } \\
\text { Ontario. }\end{array}$ & \\
\hline .87 & .000 & ..... do & Sinford, N. Y ... & \\
\hline
\end{tabular}

SUBORDER 3.

RANG 1.

SECTION 1.

Subrang 4.

(V. $5,3,1,1,4$ )

\begin{tabular}{l|l|l|l|l|}
\hline 0.76 & 0.000 & Iron ore ............. & Iron Mountain Wyu ................ & S. M. Q.. XX, 354. \\
\hline
\end{tabular}




\section{DISCUSSION OF THE DIAGRAMS.}

GENERAL DIAGRAMS.

On Pl. I are exhibited the comparative values of the chief chemical components of over 950 rocks. The relative molecular proportions of six oxides- $\mathrm{Al}_{2} \mathrm{O}_{3}$, $\mathrm{K}_{2} \mathrm{O}, \mathrm{Na}_{3} \mathrm{O}, \mathrm{CaO}, \mathrm{MgO}$, and $\mathrm{FeO}\left(=\mathrm{FeO}+2 \mathrm{Fe}_{2} \mathrm{O}_{3}\right)$-are expressed by the shapes of the individual diagrams, the silica percentages are expressed by the relative lateral positions of these diagrams, and the comparative richness in alkalies is shown by the shapes of the individual diagrams and is also expressed by their relative vertical positions.

In the upper left-hand corner of the plate a smaller multiple diagram shows the distribution of the analyses in a more concise manner, the several color's of the spots indicating the classes into which they may be subdivided, according to the quantitative system, to be noticed more fully on page 68 .

\section{VAIRIATIONS IN COMPOSITION.}

From the large diagram it is seen how manifold and intricate are the variations in chemical composition of igneous rocks. 'The larger the yellow triangles, the richer the soda and alumina; the larger the green ones, the more the potash and alumina; the taller the upper half of the individual diagram, the more the alumina; the narrower and taller the yellow and green triangles, the greater the excess of alumina over alkalies; the larger the blue triangles, the more the magnesia and lime; the larger the red triangles, the more the iron oxide and lime; the longer these lower triangles in the direction of the middle vertical line, the more the lime. Long, narrow, inclined, blue triangles indicate an excess of magnesia over lime; long, narrow, inclined, red triangles, an excess of iron oxide over lime.

The variation in the sizes of the individual diagrams, from the small ones at the left to the large ones at the right, corresponds to the great difference in silica content of the different rocks, as well as to differences in the components just named. Rocks with 75 per cent of siliea have only 25 per cent of other constituents, while rocks with 40 per cent of silica have 60 per cent of other components. Moreover, the relative number of molecules for given percentages of mass increases with the decrease in molecular woight of the component oxides. It is to be observed that rocks high in silica are relatively high in alumina and the alkalies. The molecular weights of these are: $\mathrm{Al}_{2} \mathrm{O}_{3}, 102 ; \mathrm{K}_{2} \mathrm{O} ; 94 ; \mathrm{Na}_{2} \mathrm{O}, 62$. Rocks low in silica may be high in alumina and alkalies, or high in lime, iron oxide, and magnesia. The molecular weights of the latter are: $\mathrm{FeO}, 72 ; \mathrm{CaO}, 56 ; \mathrm{MgO}, 40$. The lightness of magnesia accounts for its greater proportions in the diagrams than in the analyses. 
There are chemical variations which are somewhat regular, and others which seem irregular. First, it appears that the most siliceous rocks are rich in alumina and alkalies, and that lime increases at first more than iron and magnesia. But to this latter statement there are many exceptions.

In rocks with intermediate silica, 50 to 60 per cent, there is a gradation from those rich in alumina and alkalies and low in lime, iron, and magnesia to those rich in the latter and poor in the former. But there are rocks in this series high in alumina and lime and poor in alkalies and iron and magnesia.

The rocks low in silica, containing 35 to 50 per cent, have a wide range in variation, and attain the highest amount of magnesia. Those extremely low in silica, containing less than 30 .per cent, are very rich in iron, with little or no alumina and alkalies, and little lime or magnesia.

The diagrams of rocks with less than 28 per cent of silica are found on Pl. VII. They are not repeated on Pl. I, because of the inconvenience of a plate of such length.

If the variations are studied in greater detail it is seen that in rocks baving nearly the same silica and alkali ratios there is variation in the alumina, scarcely two rocks having the same amount. But there is more variation in the potash and soda. These values seem to be constantly shifting from place to place, with nothing suggesting a constant ratio between them. The great preponderance of soda is expressed by the predominance of yellow triangles over green ones. Dominantly potassic rocks are rare. They are more abundant among the more siliceous ones. Soda reaches its maximum in less siliceous rocks. Similar variability shows itself in the proportions of lime, iron oxide, and magnesia, whether these are subordinate or preponderant constituents.

\section{TRANSITIONS AND EXTREMES.}

It is possible to select certain shaped diagrams as types and discover many that are approximately the same. But closer inspection shows gradual variations from any two of these types to intermediate forms. And the general impression obtained from the whole assemblage is a regular variation from highly aluminous alkalic, yellow, and green figures to highly ferromagnesian calcic, red, and blue ones, combined with a very irregular variation in detail among adjacent figures.

The extremes of chemical variation reached in igneous rocks are exhibited in some of the diagrams. However, all known extremes are not expressed, because chemical analyses are wanting in several instances. Thus the extreme of pure silica attained by some intrusive dikes or veins of quartz is not represented. Chemical analyses have not yet been made of sucb rocks, or of the highly quartzose pegmatites with more than 85 per cent of silica. When they have been made the 
individual diagrams will extend to the extreme left-hand point of the multiple diagram.

Individual diagrams, composed of only a green triangle, exhibit the composition of a rock almost wholly orthoclase and quartz. One that is simply a yellow triangle is an almost pure albite rock. An almost pure nephelite rock from Ontario has been described by Adams, but analyses of it have not yet been made.

Diagrams consisting of only a narrow line represent an almost pure magnesium silicate, olivine, while those consisting of long triangles sloping to the left (Pl. VII) represent iron and titanium oxide-titaniferous magnetite.

The extremes of variability expressed in mineral terms are known to be as follows: Igneous rocks wholly quartz, almost wholly feldspar, orthoclase, albite, labradorite, and other varieties of feldspar, and those wholly nephelite; rocks almost wholly enstatite, hypersthene, olivine, magnetite. From what is known of the variable mineral composition of igneous rocks it: is clear that with more analyses to introduce into the diagrams the rariations in the shapes of the figures would be increased. Enough are shown to demonstrate the gradual character of the variations in chemical composition, and the transitions from rocks of one composition to those of another.

RELATIVE ABUNDANCE OF DIFFERENT KINDS OF ROCKS.

The distribution of the individual diagrams in the multiple one, and of the spots in the small composite diagram of Pl. I, shows that those varieties of rocks having extreme compositions, occurring in the margin of the multiple diagram, are less abundant than those having more nearly an average composition. This is still more evident when all those analyses are taken into account which were omitted because of lack of space for them. They will be found to belong to the central portion of the multiple diagram.

This is shown on Pl. VIII in a composite diagram exhibiting the variation in silica and alkali-silica ratio of 2,000 igneous rocks, which has been prepared by combining data found in this paper with those contained in the collection of analyses published by Washington, ${ }^{a}$ to whom the author is indebted for an opportunity to study the collection in proof sheets. There are more than twice as many rocks involved in this diagram as in that on Pl. I, but the results are similar, and if the spots were replaced by individual diagrams the effect would be to doubly confirm all the conclusions drawn from a study of Pl. I. The construction of such a multiple diagram, however, is not feasible at this time.

The average rock, as calculated by Clarke ${ }^{b}$ from 680 analyses of American rocks, is shown by diagram at 59.77-0.088, and that calculated by Harker ${ }^{c}$

a Op. cit.

$b$ Bull. U. S. Geol. Survey, No. 148, p. 12.

c Geol. Mag., 1899, p. 220.

$14474-$ No. $18-03-5$ 
from 397 British rocks is shown by diagran at 58.7-0.083. These diagrams resemble those of rooks having like silica and alkali-silica ratios, and occur in the densest part of the assemblage. Other averages made from greater numbers of analyses yield almost identical results. A later average, made by Clarke ${ }^{a}$ from 830 complete analyses of American rocks combined with 180 additional determinations of silica, 90 of lime, and 130 of alkalies, produce an average magma with 59.71 per cent of silica, and other constituents very nearly the same as those expressed in the diagram. Washington ${ }^{b}$ obtained a closely similar average magma from 1,811 analyses of igneous rocks from all parts of the world, having 58.24 per cent of silica. The loci of the averages of American. and of British rocks are marked by crosses on the diagram on Pl. VIII.

These prove that the average of a large number of known rocks is like the commonest rocks; conversely, that the commonest rocks are like the average of all known rocks; from which it is possible to derive all kinds of igneous rocks by processes of separation, splitting up (spaltung), differentiation. It does not follow from this that they have been produced from one common average magma, or even that they have been produced in this manner in actual fact; but such an origin is shown to be quantitatively possible and reasonable.

\section{ABSENCE OF CLUSTERING.}

A study of the diagrams of PI. I and Pl. VIII fails to detect any clustering or grouping of analyses in definite parts of the multiple diagram, such as might serve as the basis for a chemical classification of rocks. This is the same as stating that there do not appear any "natural" recognizable subdivisions of rocks on a basis of their chemical composition. Variations in seven prominent chemical factors exist throughout the system of igneous rocks, the mathematical expression of which would be extremely intricate. The study of the chemical composition of igneous rocks by graphical methods does not reveal any simple method of grouping or classifying them on a basis of their chemical constituents alone.

SIMILARITY OF ROCKS FROM DIF́FERENT PETROGRAPHICAL PROVINCES.

Further study of the diagrams and of the list of analyses shows the chemical similarity of many rocks that belong to different petrographical provinces. One illustration of this is taken from the best-known province, that of Christiania, made classic by the publications of Brögger. The diverse rocks of this region that are genetically related may be found in various places in the list and in the multiple diagrams. A comparison of the individual diagrams with those of

$a$ Clarke, F. W., Bull. U. S. Geol. Survey No. 168, 1900, p. 14.

$b$ Washington, H. S., Chemical analyses of igneous rocks from 1884 to 1900: Prof. Paper U. S. Geol. Survey No.14, 1903, p. 107. 
similar shape from other petrographical provinces will demonstrate the truth of Brögger's statement that "the same group of differentiated rocks can be produced by separation from mother magmas of quite different chemical composition." 'a A list of the chief cases of similarity is given briefly, as follows:

Tuble showing similarity between Norway rocks and those of other regions.

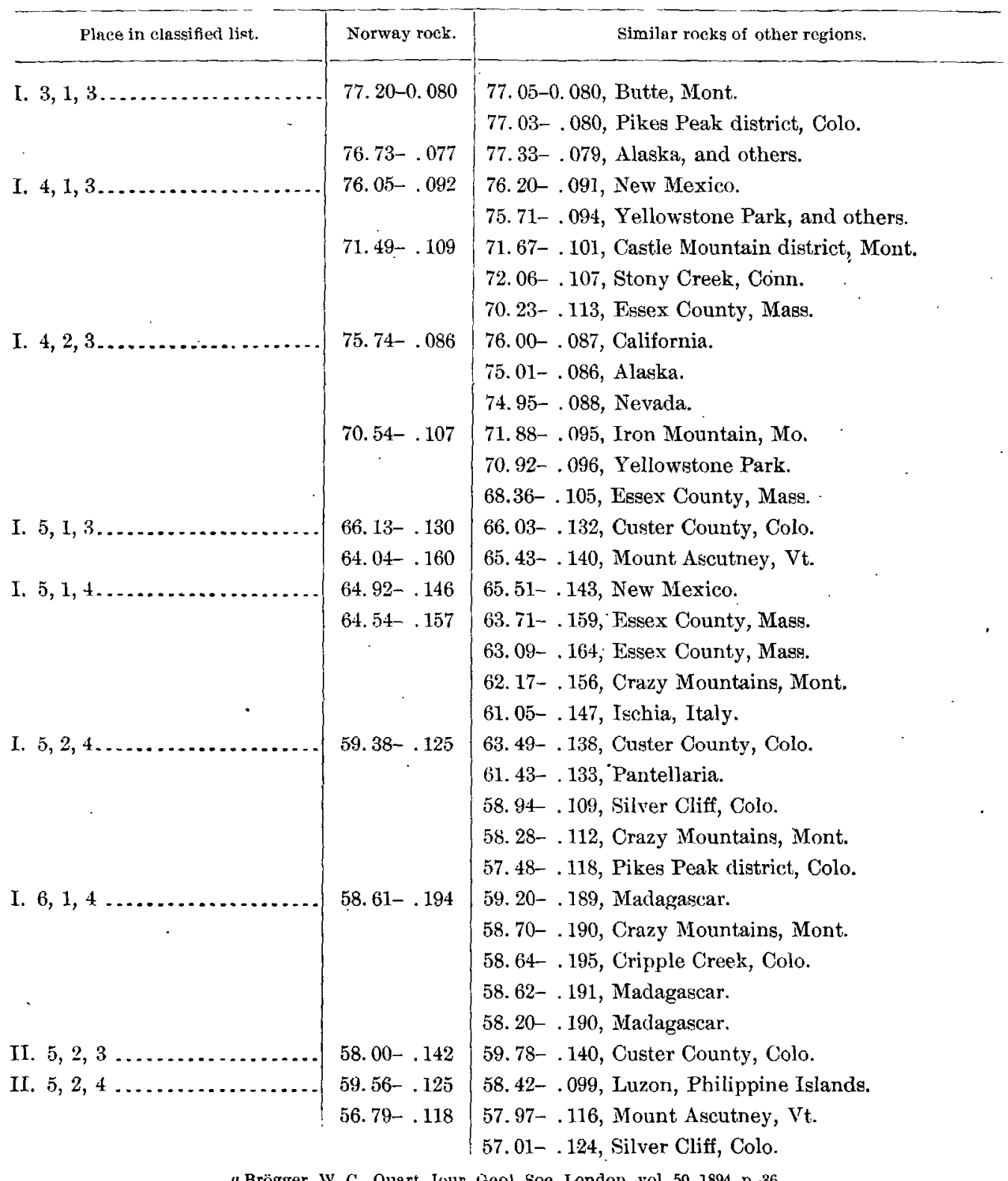


Table showing similarity between Norway rocks and those of other regions-Continued.

\begin{tabular}{|c|c|c|}
\hline Place in classified list. & Norway rock. & Similar rocks of other regions. \\
\hline II. $5,3,4 \ldots$ & $\begin{array}{l}52.91-0.119 \\
48.06-.084\end{array}$ & $\begin{array}{l}52.97-0.107, \text { Pikes Peak district, Colo. } \\
53.10-.134, \text { Madagascar. } \\
49.24-.092, \text { Ferdinandea. } \\
48.90-.090 \text {, Vosges. } \\
50.56-.079, \text { Plumas County, Cal. }\end{array}$ \\
\hline III. $5,3,4, \ldots \ldots \ldots$ & $47.90-.094$ & $\begin{array}{l}48.22-.084, \text { Mount Ascutney, Vt. } \\
47.16-.077, \text { New York: }\end{array}$ \\
\hline III. $6,3,4, \ldots \ldots \ldots \ldots \ldots$ & $43.65-.071$ & 42.03-.053, Arkansas. \\
\hline IV. $2,1,1,3,2 \ldots \ldots \ldots \ldots$. & $45.05-.030$ & $\begin{array}{l}46.56-.043 \text {, Ural Mountains. } \\
46.56-.050 \text {, Ural Mountains. }\end{array}$ \\
\hline
\end{tabular}

From this list it appears that among the various rocks, ranging from persalic to dofemic, that occur in the Christiania region there are those whose chemical composition is very similar to particular rocks found in the Ural Mountains, Vosges Mountains, Ischia, Pantellaria, Ferdinandea, Madagascar, Luzon, Vermont, New York, Massachusetts, Connecticut, Missouri, Arkansas, Colorado, New Mexico, Nevada; Yellowstone National Park, Montana, California, and Alaska. These regions belong to diverse petrographical provinces, some of them very similar to that of Christiania, but others distinctly different.

There are provinces in Finland, in Canada, and in parts of the United States closely resembling the Christiania province in the characters of their nephelitebearing rocks, but the regions are not identical in petrography. It is possible to find all degrees of similarity between petrographical provinces in various parts of the world, and the most intricate web of similar differentiation products may be woven by their comparison.

From the chemical character of igneous magmas, their content of like elements, the chemical character of the rock-making minerals, the fixed composition of some of them, and the serial variation in the composition of others, the theory of differentiation leads us to expect diversity in the rocks of all petrographical provinces of such a kind that identical or closely similar varieties of rocks will occur in otherwise unlike provinces.

\section{CLASSIFICATION OF IGNEOUS ROCKS.}

From the foregoing considerations it is evident that if we attempted to classify igneous rocks on a basis of their groupings in petrographical provinces-that is, if we should unite in the first instance rocks that are said to be genetically relatedwe should separate in the system rocks of world-wide occurrence that are alike. 
It must not be forgotten that igneous rocks are not like biological organisms, and that we are constantly misleading ourselves by the use of biological terms, such as generation, consanguinity, and family, into imagining that the relationships of various rocks should be treated-like the relationships of organisms.

When we have had more experience in the synthesis and the differentiation of molten rock magmas in chemico-physical laboratories; have handled them like other solutions of salts, which have been studied at lower temperatures; have solidified them under diverse physical conditions, we shall no doubt free ourselves from the fascination of biology and look upon rocks as the chemist looks upon a solidified mass of mixed salts. Is it not possible, in the period of preparation of such properly equipped laboratories, to anticipate their psychological effects and to think of the study of igneous rocks, their magmas and relationships, as purely physico-chemical problems, involving the measurement and comparison of mass and force, and their definite quantitative expression?

It being evident from the diagrams that there are gradual transitions in the chemical composition of igneous rocks from one extreme to another, that there are no recognizable groupings of rocks or noticeable subdivisions of the chemical series, and that chemically similar rocks occur in genetically different families, it follows that the subdivision of all igneous rocks into groups for purposes of classification must be along arbitrarily chosen lines. And the intricate character of the chemical variations, apparent in the diagrams, prevents a simple quantitative statement of any possible limits which may be selected for subdivisions of the series, however arbitrarily chosen. A careful study of the diagrams will convince one of the truth of this statement. It is a problem involving at least seven variable factors, and in its exact statement still more. It is the problem of expressing quantitatively the chemical composition of solutions of mixed salts often containing identical elements. The most artificial method of statement would be to consider the elements regardless of the possible salts in solution. A less artificial method would seem to be one which takes into account the salts which may enter the solution or may separate from it. In rock magmas the salts in solution may properly be considered to have the composition of those minerals which separate and crystallize when the magmas solidify. But it is understood that salts in solution may become split up into molecules of simpler composition, may be partially dissociated into ions, and may be combined in more than one manner in the act of separating from solution under diverse conditions.

When it is remembered that igneous rocks are solidified and for the most part crystallized mixed solutions, the expression of their chemical composition in terms of the separated salts or minerals developed in them is eminently reasonable. These principles underlie the quantitative classification of igneous rocks proposed by Cross, Pirsson, Washington, and the author. 
In expressing the chemical composition of the rocks in terms of the minerals developed it is advisable to reduce the complex possibilities by selecting the simpler and commoner minerals as standards of comparison. The details of the method must be learned from the published essay, ${ }^{a}$ to which the reader is referred if he is not already familiar with it.

\section{CLASSIFIED DIAGRAMS.}

The resulting chemical subdivision and grouping of igneous rocks by the method of quantitative classification recently proposed can be well shown graphically by the diagrams already described: The clearest exposition, however, requires a greater number of multiple diagrams than has been prepared, in order to separate all the divisions established by the classification. But the expense of publication has prevented the carrying out of such a plan, and so fewer multiple diagrams have been used, with a consequent loss of simplicity and a greater complexity than exists in the actual system of classification itself. The advantages to be derived from the exposition made, however, are sufficient to warrant its publication. It presents the salient features and results of the classification in a manner which can not be conveyed by words.

Multiple diagrams have been prepared to exhibit the grouping and variations in rocks belonging to the five classes of the quantitative system. Those of the first, second, and third classes are represented on three separate diagrams, while those of Classes IV and V are placed on one diagram because of their smaller number. The diagrams are presented in two forms - in one form spots represent the distribution of loci of analyses and their range of variation with respect to silica and alkalies; the other form is an individual diagram showing the variations with respect to the principal chemical components. (Pls. III, IV, V, VI, and VII.)

The distribution of the analyses belonging to the five classes, with respect to silica and alkalies, is shown by the spotted diagrams on Pls. III, IV, and V and by the differently colored "spots in the composite diagram on PI. I, in which the yellow spots belong to Class I, the red spots to Class II, the blue ones to Class III, and the black ones to Classes IV and V.

Since Class $I$ is persalic, that is, is extremely rich in salic minerals-quartz and aluminous feldspathic minerals-most of these rocks occur in the diagram near the curved lines showing the positions of rocks composed wholly of quartz with albite or orthoclase, and albite with nephelite, or orthoclase with leucite. But rocks of this class include all those rich in alumina, which may form anorthite, a nonalkalic mineral. Consequently they also occur in the diagram remote from the curved lines, approaching the locus of anorthite at 43.160.-0.0.

a Quantitative classification of igneous rocks. Chicago, 1903. Also Jour. Geol., vol. 10, 1902. 
Class II is dosalic, that is, dominantly salic. There are less feldspathic minerals and more ferromagnesian ones, and since the latter are mostly low in alkalies, rocks of this class occur in the diagram farther from the limiting alkalifeldspathic curves than those of Class I.

As Class III is still richer in ferric minerals and lower in alkalies, rocks of this class occur still more removed from the curves just mentioned. The same is true to a greater degree for rocks of Classes IV and V.

It is seen that the positions of these classes of rocks overlap one another more or less in the composite diagram on Pl. I, hence the confusion of individual diagrams in the large multiple diagram of Pl. I.

\section{DIAGRAMS OF CLASS I.}

Let us consider separately the diagrams of the different classes of rocks, beginning with Class I. The persalanes, represented by 412 individual diagrams, being characterized by preponderant salic ninerals, are subdivided into nine orders, according to the proportions of quartz and feldspars and of feldspars and feldspathoids (lenads). The order highest in quartz, perquaric, is highest in silica, and that highest in lenads, perlenic, is lowest in silica. Consequently the nine orders follow one another from left to right in the multiple diagram (Pls. II and III). The limits of the orders may be shown graphically for extreme cases, and may be interpolated for intermediate ones. Owing to the difference in the molecular weight of sodium and potassium, the limits for orders based on quartz and soda-feldspar (albite) and on quartz and potash-feldspar (orthoclasej) do not coincide. Further, the greater chemical difference between the soda-lenad, nephelite, an orthosilicate, and the potash-lenad, leucite, a metasilicate, causes a still wider divergence between the boundaries of the orders based on albite and nephelite on the one hand and on orthoclase and lencite on the other. That is to say, the limits between the nine orders of persalic rocks vary according as the alkalies in the rocks are soda or potash. This is shown by the diagram on Pl. II. The black lines nearly at right angles to the upper curve mark the limits of the nine orders for ideal rocks composed wholly of quartz, albite, anorthite, or of soda-nephelite, albite, anorthite. The red lines transverse to the upper red curve mark the limits of the nine orders for rocks composed wholly of quartz, orthoclase, anorthite, or of leucite, orthoclase, anorthite.

The division lines for soda orders start from points on the quartz-albite-nephelite curve situated where $q: a b:: 7: 1 ; q: a b:: 5: 3 ; q: a b:: 3: 5 ; q: a b:: 1: 7 ;$ ab: ne ::7:1; ab: ne::5:3; ab:ne::3:5; ab:ne::1:7, and run to points on the quartz-anorthite line and the anorthite-nephelite line were $q:$ an $:: 7: 1 ; q:$ an $:: 5: 3 ; q:$ an ::3:5; $q:$ an :: $1: 7$; an: ne:: $7: 1$; an: ne::5:3; an:ne::3:5; an: ne::1:7. The same may 
be said of the division lines for potash orders, substituting the names of orthoclase and leucite for those of albite and nephelite, respectively.

The diagram on Pl. II shows that the first four orders in each case nearly coincide, the boundary lines for potash rocks lying a little to the right of those for soda rocks. That is, the limit for each quaric order of potash rocks reaches a little lower silica than that for the corresponding orders of soda rocks.

The diagram also shows that the last four orders in each case differ more and more widely as they become more lenic. The range for the potash rocks is much more restricted than for the soda rocks, and the limits of orders for the potash rocks are higher in silica than for the corresponding soda rocks. This is because the potash lenad, leucite, is a metasilicate with 55.0 per cent $\mathrm{SiO}_{2}$, while the soda lenad, nephelite, is an orthosilicate with 42.3 per cent $\mathrm{SiO}_{2}$.

Since most rocks contain both soda and potash, the limits for the orders will occur between the extremes illustrated on Pl. II, varying with the ratio between soda and potash in each case. This explains the confused arrangement of the lenic orders in the upper right-hand portion of the diagram of persalic rocks on Pl. III.

In this diagram the number of the order of each rock is given by the first figure under its individual diagram. The number of the rang is given by the second figure. A study of these numbers will show that in the left-hand portion of the large diagram there is general concordance among the individual diagrams with respect to orders and rangs, except near the borders between orders, and between rangs. 'But in the right-hand upper portion of the large diagram there is great confusion of orders. It will be noted that where diagrams of different orders occur close together that with the higher number is the more potassic, other things being equal. The confusion would disappear if diagrams were constructed to exhibit the various subrangs. The correspondence between the distribution of the persalic rocks according to orders in the multiple diagram on Pl. III and the theoretical limits of the orders as shown on Pl. II is apparent. The orders form belts transverse to the curves which are the limits to ideally pure alkalic persalic rocks, namely, those consisting wholly of quartz, alkali-feldspars, or lenads.

As the wholly alkalic rocks occur along the limiting curves just mentioned and those free from alkali occur on the bottom line of the diagram, it follows that the various rangs of persalic rocks occur at intervals between these limiting lines; the peralkalic nearest the upper curves, the percalcic nearest the bottom line.

The boundary lines between the 5 rangs of persalic rocks are shown in the diagram on Pl. II. The black lines nearly parallel to the upper curve mark the limits for those based on soda alone; the red lines for those based on potash alone. Since most rocks contain both alkalies the limit for rangs will shift according to the ratio between soda and potash in the rocks. The rangs form belts roughly parallel to the limiting curves and bottom line of the multiple 
diagram. The limiting lines are drawn from the point of pure quartz, where they all become zero, to points on lines marking the middle of the perfelic order, 5-that is, the lines for mixtures of albite and anorth:e in the case of pure soda rocks, and that for mixtures of orthoclase and anorthite for pure potash rocks. The points on these lines occur in the first case where $\mathrm{Na}_{2} \mathrm{O}: \mathrm{CaO}: \mathrm{T}: 1$; $\mathrm{Na}_{2} \mathrm{O}: \mathrm{CaO}:: 5: 3 ; \mathrm{Na}_{2} \mathrm{O}: \mathrm{CaO}:: 3: 5 ; \mathrm{Na}_{2} \mathrm{O}: \mathrm{CaO}:: 1: 7$. They ouvur in the second case where $\mathrm{K}_{2} \mathrm{O}: \mathrm{CaO}: 7: 1$, etc. From these points the limiting lines pass to corresponding points on lines representing mixtures of nephelite and anorthite and of leucite and anorthite.

From Pl. II it is seen that the range of rangs is nearly concordant in the quaric orders of persalic rocks and more and more div zent in the more lenic orders according as the rocks compared are more sodic or more potassic. This explains the apparent confusions of rangs in the right-hand portion of Pl. III.

There is a close correspondence between the distribution of the persalic rocks according to rangs in the large diagram (Pl. III) and the theoretical limits of rangs shown in Pl. II. This is shown by the second figu"e under each individual diagram.

The diagram on Pl. II exhibits the limitations of orders and rangs of the persalic rocks containing lenads. These are seen at the right end of the diagram. The limiting lines representing combinations of nephelite and anorthite and of leucite and anorthite cut diagonally across the belts of orders and rangs. Thus in soda rocks whose limits are shown by black lines order 9 has only rang 1; order 8 contains rangs 1 and 2; order 7 embraces rangs 1, 2, and 3; order 6 has four rangs-1, 2, 3, and 4, while all five rangs occur in order 5. In potash rocks, represented by red lines, the conditions are not quite so simple, as is seen in the diagram. Each of the lenic orders has a little larger range in calcic content and includes a small amount of a more calcic rang than occurs in the purely soda rocks.

There are variations in composition in rocks not yet taken into consideration which further modify the distribution and arrangement of we individual diagrams. Most rocks contain femic components, and these are generally low in alkalies or free from them. With increasing amount of nonalkalic femic components the limits of rangs in the multiple diagram shift downwart, for rangs are established on the ratio between salic alkalies and salic lime-that is, between the $\mathrm{K}_{2} \mathrm{O}$ and $\mathrm{Na}_{2} \mathrm{O}$ and the $\mathrm{CaO}$ in the standard feldspars and lenads. Consequently the more femic minerals present, the less salic, and the smaller the alkalies necessary to maintain a given ratio with diminished salic lime. Hence the boundary line between rang 1 and rang 2 occurs further below the limiting quartz-feldspar lenad curves the greater the amount of nonalkalic femic components in rocks. This is evident on comparing rangs in Pls. III and IV. 
In the majority of rocks in which there is more alumina than alkalies there is usually more lime than is sufficient to satisfy the excess of alumina, so that the ratio between salic alkalies and salic lime, which determines the rang, is dependent on the amount of alumina in the rock. From this it happens that rocks having like silica percentages and alkali-silica ratios, located near one another in the multiple diagram, may differ in rang because of differences in alumina. Other things being equal, those with higher alumina will have lower ratios between salic alkalies and salic lime; and conversely, those with lower alumina will have higher alkali-calcic ratios. And these differences may affect the rang of the rocks compared. Examples of this kind are more evident in diagrams of rocks of Class $\mathrm{II}$, and are found at 56.3-0.065 and 56.7-0.066, also 56.6-0.074 and 56.9-0.074, on Pl. IV.

Where the femic components of persalic rocks are alkalic the rocks are always in peralkalic rang 1, so that the limit of rang 1 is extended downward in the multiple diagram. The individual diagrams for rocks of this kind are characterized by comparatively short $\mathrm{Al}_{2} \mathrm{O}_{3}$ lines, less than the sum of $\mathrm{K}_{2} \mathrm{O}$ and $\mathrm{Na}_{2} \mathrm{O}$.

The effect of increasing amounts of femic components in persalic rocks upon the limits of orders is not so easily appreciated, the relations being less simple. If we compare two rocks with like silica and alkali percentages, which would occur near one another in the diagram (Pl. III), the more nonalkalic femic components the less salic; that is, the less alumina and the less anorthite. If the rocks are quaric the ratio between quartz and feldspar is increased by decreasing the anorthite feldspar. But the substitution of $\mathrm{FeO}, \mathrm{MgO}$, or $\mathrm{CaO}$ for $\mathrm{Al}_{2} \mathrm{O}_{3}$ would require more $\mathrm{SiO}_{2}$ to form metasilicates than that required to form anorthite, consequently the free $\mathrm{SiO}_{2}$, quartz, would be reduced, but to a smaller amount than the feldspar is reduced, consequently the ratio between the quartz and feldspar would be higher in the rock with more nonalkalic femic components. The limits between the quaric orders would shift to the right.

If the rocks are lenic, containing nephelite, sodalite, or leucite, a reduction of anorthite feldspar would increase the ratio between lenads and feldspars. A substitution of $\mathrm{FeO}, \mathrm{MgO}$, or $\mathrm{CaO}$ for $\mathrm{Al}_{2} \mathrm{O}_{3}$ in amount equal to the $\mathrm{CaO}$ of presupposed anorthite would necessitate their conversion to metasilicates according to the method of the quantitative classification under discussion. This would require more silica than is required by anorthite, so that the amount of alkali feldspars would be reduced and the lenad-feldspar ratio further increased. In case the lime were replaced by magnesia and iron oxide and converted into orthosilicates there would be slight change in the silica available for the salic components, because of the general preponderance of magnesia in such rocks, and its lighter molecular weight and consequent greater number of molecules. Hence, 
in most cases an increase of femic components would increase the lenad-feldspar ratio, and the limits between lenic orders of persalic rocks would shift toward the left.

In the less frequent cases in which alkali femic components occur, the limits between all orders shift to right or left according to the relative proportions of the alkali-femic components and according as they are acmite or are free from ferric iron.

The occurrence of magnetite, ilmenite and other mitic components also modifies the position of the ordinal limits, but in peralkalic rocks these constituents generally occur in such small amounts that their effects may be disregarded in the interpretation of the diagram under consideration.

With respect to subrangs it may be said that in the great majority of cases they can be recognized by a comparison of the yellow and green triangles representing the relative amounts of soda and potash in the rock. For in most instances all of the alkali is salic. In other cases where part of it is femic, the ratio between the salic alkalies can be made out by a scrutiny of the triangles and a comparison of the amounts of alkalies and alumina expressed by the lengths of the potash, soda, and alumina lines. The ratio in this case would be

$$
\frac{\mathrm{K}_{2} \mathrm{O}}{\mathrm{Al}_{2} \mathrm{O}_{3}-\mathrm{K}_{2} \mathrm{O}}
$$

In general, where alumina is in excess of the sum of the alkalies, a comparison of the triangles named, or of the lengths of the potash and soda lines, will determine the subrang to which the rock belongs. Those in which $\frac{\mathrm{K}_{2} \mathrm{O}}{\mathrm{Na}_{2} \mathrm{O}}>\frac{7}{1}$ are in perpotassic subrang 1 ; those with $\frac{\mathrm{K}_{2} \mathrm{O}}{\mathrm{Na}_{2} \mathrm{O}}<\frac{7}{1}>\frac{5}{3}$ are dopotassic subrang 2; when $\frac{\mathrm{K}_{2} \mathrm{O}}{\mathrm{Na}_{2} \mathrm{O}}<\frac{5}{3}>\frac{3}{5}$ the subrang is 3 , sodipotassic; when $\frac{\mathrm{K}_{2} \mathrm{O}}{\mathrm{Na}_{2} \mathrm{O}}<\frac{3}{5}>\frac{1}{7}$ it is dosodic, subrang 4 ; and with $\frac{\mathrm{K}_{2} \mathrm{O}}{\mathrm{N} \mathrm{a}_{2} \mathrm{O}}<\frac{1}{7}$ the subrang is 5 , persodic.

These divisions apply only to the first three rangs. In the fourth rang, which is docalcic, there are only three subrangs, namely: $1, \frac{\mathrm{K}_{2} \mathrm{O}}{\mathrm{Na}_{2} \mathrm{O}}>\frac{5}{3}$, prepotassic; 2, $\frac{\mathrm{K}_{2} \mathrm{O}}{\mathrm{Na}_{2} \mathrm{O}}<\frac{5}{3}>\frac{3}{5}$, sodipotassic; $3 ; \frac{\mathrm{K}_{2} \mathrm{O}}{\mathrm{Na}_{2} \mathrm{O}}<\frac{3}{5}$, presodic. The fifth rang being precalcic, subrangs are not recognized.

A study of the diagrams on Pl. III shows that soda preponderates over potash in most persalic rocks; that sodipotassic rocks, subrang 3, are the most numerous; that dopotassic rocks are rare, and perpotassic ones extremely so. The numbers of rocks in the 5 rangs are $3,31,201,156,21$. 
Variations in the femic components, $\mathrm{FeO}, \mathrm{MgO}$, and femic $\mathrm{CaO}$, are noticeable in the individual diagrams and represent differences among the subordinate mineral constituents of the rocks. Their quantity is so small that they may be neglected in the magmatic classification of these persalic rocks.

The diagrams plotted together demonstrate the gradual transition between orders of rocks of Class I, as well as between rangs and subrangs. And since any individual diagram may represent a large body of igneous rock or a small facies of one, and since any of the diagrams may represent rock bodies of considerable importance, it follows that all parts of the series may be of equal importance geologically or petrologically, and consequently rocks that happen to belong on the border line of any set of subdivisions into which the whole rock series must be divided, for purposes of classification of any kind, may be as important as those situated at the center of such an arbitrary division, and in some regions it will happen that such rocks are the most important.

When the student has become convinced of the continuity of the chemical series of igneous rocks he will not be surprised to discover that a rock of great importance belongs on the boundary of a classificatory division, or probably falls on both sides of a division line. It will not affect the importance of the rock or militate against the value of the system of classification. It will simply be a "natural" fact. And the system of classification will appear to him as "natural" as any other system which attempts to classify igneous rocks.

DIAGRAMS OF CLASS II.

The multiple diagram of 335 dosalic rocks of Class II (Pl. IV) may be readily interpreted by comparison with that of Class I (PI. III). The spotted diagram to the left shows the distribution of the analyses with respect to silica and alkalies. The rocks are for the most part lower in alkalies than those of Class I, because the femic components are generally nonalkalic. The few'cases with rather high alkalies contain acmite molecules, which fact is indicated in the individual diagrams by pronounced iron oxide and soda and by low alumina. Several of these occur in the left-hand part of the multiple diagram at 74.35 $0.092,69.02-0.121$, etc.

The dominant constituents in dosalic rocks being the same as those in the persalic rocks, nine orders have been established, in the same manner as in Class I. Rangs and subrangs also correspond to those in Class I. So there is the same general arrangement of individual diagrams in PI. IV, according to orders and rangs, as in the multiple diagram of Class I, with the modifications produced by increasing amounts of femic components, for the most part nonalkalic. The belts of orders are transverse to the limiting quartz-alkali-feldspar lenad, curves. The limits of 
rangs are lower than in the diagram of Class $I$. There is the same confusion of orders and rangs along the division lines consequent upon variations in alumina and femic components, and a like diversity of alkalies, shown by the yellow and green triangles, with a strong preponderance of soda over potash. The greatest number of rocks are dosodic. The numbers in each rang are: $0,17,126,183,9$.

With an increase in the femic components the variations in iron oxide, magnesia, and lime become more noticeable and important, and it is necessary to recognize distinctions among the subordinate femic components, that is, to note grads and subgrads. The nature of the grads can not be determined directly from the diagrams because it depends on the amount of available silica, which may be combined with femic bases to form silicates, pyroxene, olivine, or akermanite, and also on the amount of ferric oxide and titanium oxide. The ferric oxide is blended with ferrous oxide in the diagrams and is not indicated graphically. Titanium oxide is so small in most dosalic rocks that it is not introduced into the diagrams in this Class II.

It is to be noted, however, that the silica is sufficiently high to form silicates of almost all the femic bases, and ferric oxide is in most cases low, so that the great majority of dosalic rocks belong to the prepolic grad 1, that in which the femic silicates-pyroxene and olivine-preponderate over the femic nonsilicates-magnetite, hematite, ilmenite, rutile, and the silico-titanate, titanite.

As to the subgrad to which these rocks belong, by far the largest number contain ncnalkalic femic components, and therefore fall in the premirlic subrang 1 . A small number contain alkalic femic components in nearly the same proportions as nonalkalic ones, and fall in the alkalimirlic subrang 2 . Some, characterized by highly alkalic femic constituents, such as acmite, belong to subrang 3.

The multiple diagram for Class II (Pl. IV) exhibits similar transitions between orders and between rangs as those shown by the diagram for Class I, and permits the same conclusions to be drawn from it. The chemical series will appear more and more. complete as more varieties of rocks are analyzed.

\section{DIAGRAMS OF CLASS III.}

The chemical relations of the salfemic rocks, Class III, are expressed graphically on Pl. VI. Two of them are so high in silica that they have been plotted on Pl. IV with rocks of Class II. One is rockallose, with $73.60 \mathrm{SiO}_{2}, 0.091 \frac{\text { alk. }}{\mathrm{SiO}_{2}}$. It is intermediate between Classes III and II. The other is a pantellerite, with 68.75 $\mathrm{SiO}_{2}, 0.144 \frac{\text { alk. }}{\mathrm{SiO}_{2}}$. It is also intermediate between Classes III and II.

The distribution of the rocks with respect to silica and alkalies is shown in the upper diagram of $\mathrm{Pl}$. V. The location of the analyses in the multiple diagram is still farther from the limiting curves than in Class II; and the silica 
ranges lower. The alkali-silica ratios reach high values in some cases. $\mathrm{TiO}_{2}$ is present in notable amount in a number of instances.

It will be observed that the lower half of the individual diagrams is the larger in nearly every case, but the long, narrow, upper triangles indicate that there is more alumina than alkalies, and that part of the lime is salic, so that the salic components are more nearly equal to the femic than appears at first glance.

There are but two rocks in quarfelic order 3 , those mentioned above, and plotted on Pl. IV. Very few occur in order 4 . The greatest number are perfelic, order 5, and the lenic orders are well represented.

Most of the perfelic rocks are alkalicalcic, rang 3 , or docalcic, rang 4 . The few that are peralkalic or domalkalic are low in alumina, as, for example, 54.2-0.160 and $45.2-0.085$. It is noticeable also in the lenic salfemanes, orders $6,7,8$, and 9, that the domalkalic character of the salic minerals in a number of rocks is due to low alumina rather than to high alkalies, as $50.00-0.110,46.00-0.121$, and $42.7-0.139$.

There are great variations in the shapes of the diagrams of rocks nearly alike in silica and alkali-silica ratio. A few rocks are rich in potash, notably those from Leucite Hills, Wyoming, 54.08-0.163, and others. A greater number are rich in soda, occurring in the upper part of the multiple diagram. But the majority are sodipotassic and dosodic.

Since in these rocks the femic minerals are equal or nearly equal to the salic, and in some cases are more abundant, they are as characteristic of the rocks as the salic minerals. The salfemanes differ considerably with respect to these constituents, which is plainly shown by differences in the shapes of the lower part of the individual diagrams. Some are long in the direction of lime; others long in magnesia. Few have more iron oxide than magnesia. From this it follows that the grad, subgrad, and section of subgrad are as important in the classification of these rocks as are order, rang, and subrang.

The grad of these rocks can not be determined from the diagrams because it depends upon the proportions of ferromagnesian silicates and nonsilicates determined by the available silica and the ferric iron not allotted to acmite. It is to be remarked that the great majority of salfemic rocks included in the diagrams are dopolic, grad 2-that is, normative pyroxene and olivine dominate over magnetite, hematite, and ilmenite. In most of these rocks there is a notable amount of normative nonsilicates. The majority of these rocks are perpyric or dopyric, sections 1 and 2-that is to say, normative pyroxene preponderates over olivine.

The character of the subgrad is indicated by the diagrams, since this depends upon the proportion of alkalies that enter the femic minerals. With few excep- 
tions the alumina is greater than the sum of the alkalies, so that the femic minerals are nonalkalic and the rocks belong to the permirlic subgrad 1 . The exceptions to this are the two rocks rich in acmite-rockallose and the rock near varingose.

The diagrams also show that most of these salfemanes are domiric, section 2, ferrous oxide and magnesia dominating over femic lime. This, however, is not perfectly evident in the diagrams because of the lack of information concerning ferric iron and the necessity of deducting salic lime from the total lime.

Finally, when it is remembered that nearly half the molecules of iron oxide belong to ferric oxide, the diagrams show that in most cases the rocks are domagnesic, subsection 2, with magnesia dominant over ferrous iron. In a few instances they are permagnesic, which is indicated by long, narrow, blue triangles.

DIAGRAMS OF CLASSES IV AND V.

The diagrams of dofemic and perfemic rocks, Classes IV and V, are plotted together on Pl. VII. This has been done because of the comparatively small number of analyses of these rocks at hand and the desire to economize space. The distribution of these rocks with respect to silica and the alkalies is shown in the lower diagram on Pl. V. As should be expected, these rocks are far from the quartzalkali-feldspathic curve, are low in alkalies, and extend to the lowest limit of silica. The diagram of one rock comparatively high in alkalies falls outside the area of Pl. VII, and is not represented. The rock is a nephelite-basalt from Katzenbuckel, Odenwald (45.04-0.209). Individual diagrams belonging to rocks of Classes IV and $\mathrm{V}$ are designated by Roman numerals, IV and V, before Arabic ones denoting orders and sections of orders.

The arrangement of the individual diagrams in the multiple one is in accord with the general system adopted with special reference to the great majority of rocks, the more salic ones. The rocks of Classes IV and V are less than one-tenth of all that are represented in the diagrams. Their arrangement is related to one component of prime importance, silica. But alkalies are extremely subordinate and would not properly be chosen as a basis for their coordination were these rocks considered by themselves. However, their relations to the more salic rocks are exhibited by retaining the same system of arrangement.

The chief difference between the rocks of Classes IV and V lies in the amount of alumina, with which in general there is sufficient lime or alkalies to form salic feldspars or lenads. In one case only is the composition of the magma such as to require the assumption of salic corundum, the rock being magnetite-spinelite from Routivare, Sweden (4.08-0.092). Most all of the rocks containing alkalies belong to Class IV, but this class also embraces rocks without alkalies, the diagrams occurring 
on the zero line for alkali-silica ratios. Nearly all the rocks of Class $V$ are so low in alkalies as to occur on the zero line. The higher ratios obtaining in several iron ores with 4 to 8 per cent of silica may easily be due to errors in chemical analysis, though, of course, they may be correct values.

Since dofemic and -erfemic rocks are divided into orders according to the relative proportions of femic silicates and nonsilicates, and into sections of orders according to the proportions of femic metasilicates and orthosilicates, it follows that the five orders and sections range according to the silica content of the rock from perpolic order 1, perpyric section 1, with highest silica, 55.5, to permitic order 5, with lowest silica, 0.7 . In like manner the various sections under each order range from higher to lower silica. These distinctions do not appear in the shapes of the diagrams, but in their lateral position in the multiple diagram.

With respect to rangs it happens that without exception all the rocks represented in this collecwon of analyses belong to permirlic rang 1, the alkalies which occur in the rocks being salie, which is shown in the diagrams by the alumina line exceeding the sum of those of the alkalies.

Sections of rangs and subrangs are based on the proportions of femic lime, magnesia, and ferrous, oxide, but the proportions of ferrous and ferric oxide are not indicated in the diagrams. It is to be noted, however, that iron is generally subordinate to magnesia in most perfemic rocks with more than 28 per cent of silica, so far as they are represented in the collection of analyses; and when it is remembered that a part of this iron is ferric, it will be seen that the proportion of ferrous iron is smaller than appears in the diagrams.

While the lime is considerable in a large number of cases, it is very small in many of the rocks, and when salic lime is deducted it is found that the femic lime is subordinate to the magnesia and ferrous iron in nearly every instance. A majority of the dofemic rocks, Class IV, are domiric, section 2 of rang 1. A smaller number are permiric, section 1 of rang 1 . In nearly every case these rocks are domagnesic, subrang 2. The iron ores of Class IV are perferrous, subrang 5 .

The perfemanes, Ciass V, with the exception of the iron ores, are permiric and domiric in about equal numbers. Lime is subordinate to magnesia and iron. They belong to sections 1 and 2 of rang 1 . In nearly every case these rocks are permagnesic, subrang 1 . This is shown by the elongation of the magnesia line. The iron ores of course are perferrous, subrang 5. The abrupt change from the titaniferous iron ores, below 27 per cent silica, to the magnesium-rich rocks above this percentage of silica is very noticeable. It indicates that the intermediate transitional rocks which have been described by petrographers have not been analyzed. 
The diagrams of the different classes of igneous rocks established by the quantitative system illustrate the chemical characters of the rocks grouped together by this system, and show the degrees of similarity and the ranges of variation within the different divisions of the classification so far as they exist in the rocks represented by the 977 analyses in the collection.

A greater number of multiple diagrams representing separate divisions of the system. will give more definite graphic expression to these minor groups of rocks. Their preparation and publication at this time have not been feasible. It is possible, also, to devise individual diagrams which shall express more or less definitely the norm of each rock, but these would of necessity be so intricate that a larger scale of drawing would be required, entailing serious difficulties of publication.

It is believed that the simpler individual diagrams and more composite multiple ones here presented will serve a better purpose at this time in demonstrating graphically some of the chemical facts and relationships that have been used by Crose, Pirsson, Washington, and the author as the foundation on which they have constructed the quantitative system of rock classification.

\section{CORRELATION OF IGNEOUS ROCKS CLASSIFIED BY THE QUANTITATIVE AND QUALITATIVIE SYSTEMS.}

The classified list of rocks already given shows the character of the correlation that may be made of the tẉo systems of classification. The variety of names that appeai within any one of the more. common divisions of the quantitative systems is due in part to differences in chemical composition, which. are recognized by subdivisions of the system of a lower order, as grad and subgrad. It is in part due to diversity of texture and mineral development, which is expressed in this system by terms qualifying the magma names. But it is also due to a great extent to essential differences in the bases of classification in the two systems and to the flexibility of qualitative definitions.

A brief tabulated statement of the salient features of the correlation is given, with some general remarks concerning the more common relationships. Greater detail must be gotten from the classified list and from the collection of rock analyses published by Washington.

$$
\text { 14474-No. 18-03- -6 }
$$


CLASS I. PERSALANE.

\begin{tabular}{|c|c|c|c|c|c|c|c|c|}
\hline 1. Victorare. & 3. Belgare. & 3. Columbare. & 4. Britannare: & 5. Canidare. & 6. Russare. & 7. Tasminare. & 8. Ontarare. & 9. $\longrightarrow$. \\
\hline Quartz-rcins. & Quartz-pegmatite. & \multicolumn{2}{|c|}{ Granite. } & $\begin{array}{l}\text { Syenite, anortho- } \\
\text { site. }\end{array}$ & \multicolumn{3}{|c|}{ Nephelite-syenite. } & $\begin{array}{l}\text { Nephelite-pegma- } \\
\text { tite. }\end{array}$ \\
\hline$\cdot$ & $\begin{array}{l}\text { 1. 1. Dargase. } \\
\text { Quartz-pegmatite. }\end{array}$ & $\begin{array}{l}\text { R. 1. Alaskase. } \\
\text { Aplite. } \\
\text { Granite. } \\
\text { Alaskite. } \\
\text { Tordrillite. } \\
\text { Quartz-porphyry, } \\
\text { Quartz-kera to- } \\
\text { phyre. } \\
\text { Rhyolite. } \\
\text { Obsidian. } \\
\qquad \\
\text { R. .. Alsbnchase. } \\
\text { Aplite. } \\
\text { Granite. } \\
\text { Alsbachite. } \\
\text { Rhyolite. }\end{array}$ & $\begin{array}{l}\text { R. 1. Liparase. } \\
\text { Aplite. } \\
\text { Grunite. } \\
\text { Quartz-syenite. } \\
\text { Syenite-porphyry. } \\
\text { Grorudite. } \\
\text { Quartz-panteller- } \\
\text { ite. } \\
\text { Rhyolite. } \\
\text { Puisanite. } \\
\text { Comendite. } \\
\text { Quartz-ke r a to- } \\
\text { phyre. } \\
\text { Obsidian. } \\
\text { R. 2. Toseanase. } \\
\text { Aplite. } \\
\text { Granite. } \\
\text { Quartz-monzonite. } \\
\text { Granodiorite. } \\
\text { Diorite. } \\
\text { Syenite. } \\
\text { Quart-syen i te- } \\
\text { porphyry. } \\
\text { Rhyolite. } \\
\text { Toseanite. } \\
\text { Latite. } \\
\text { Dellenite. } \\
\text { Dacite: } \\
\text { Trachyte. } \\
\text { Andesite. }\end{array}$ & \begin{tabular}{|l|} 
R.1. Nordmarkase. \\
Syenite. \\
Quartz-syenite. \\
Neph.-syenite. \\
Nordmarkite. \\
Pulaskite. \\
Umptekite. \\
Albitite. \\
Sölysbergite. \\
Keratophyre. \\
Bostonite. \\
Trachyte. \\
Phonolite. \\
\\
R. g. Pulaskise. \\
Quurtz-syenite. \\
Neph.-syenite. \\
Pulaskite. \\
Nordmarkite. \\
Laurvikite. \\
Sodalite-syenite. \\
Diorite. \\
Bostonite. \\
R h om ben por- \\
phyry. \\
Vulsinite. \\
Keratophyre. \\
Tracbyte. \\
Leucite-trachyte. \\
Andesite.
\end{tabular} & $\begin{array}{l}\text { R. 1. Mlaskase. } \\
\text { Neph.-aplite. } \\
\text { Neph.-syenite. } \\
\text { Foyaite. } \\
\text { Ditroite. } \\
\text { Miascite. } \\
\text { Mariupolite. } \\
\text { Tinguaite. } \\
\text { Heronite. } \\
\text { Phonolite. } \\
\text { Leucite-phonolite. } \\
\\
\text { R. .. Vlezzenaso. } \\
\text { Neph.-syenite. } \\
\text { Neph.-r homben } \\
\text { porphyry. } \\
\text { Neph.-tinguaite. } \\
\text { Tinguaite. }\end{array}$ & $\begin{array}{l}\text { R. 1. Laugenise. } \\
\text { Foyaite. } \\
-\end{array}$ & $\begin{array}{c}\text { R. 1. } \\
\text { Neph.-syenite. }\end{array}$ & $\begin{array}{c}\text { R. 1. } \\
\text { Neph.-pegmatite. }\end{array}$ \\
\hline
\end{tabular}


CLASS I. PERSAIAANE-Continued.

\begin{tabular}{|c|c|c|c|c|c|c|c|c|}
\hline 1. Vietorare. & 2. Belgare. & 3. Columbare. & 4. Britannare. & 5. Canadare. & 6. Russure. & 7. Tasmanare. & 8. Ontarare. & 0. - \\
\hline Qun rtz-veins. & Quartz-pegmatite & \multicolumn{2}{|c|}{ Granite. } & $\begin{array}{l}\text { Syenite, anortho- } \\
\text { site. }\end{array}$ & \multicolumn{3}{|c|}{ Nephelite-syenite. } & $\begin{array}{c}\text { Nephelite-pegmia } \\
\text { tite. }\end{array}$ \\
\hline ' & - & $\begin{array}{l}\text { R. 3. Rlesenase. } \\
\text { Granite. } \\
\text { Quartz-diorite- } \\
\text { porphyry. } \\
\text { Toscanite. } \\
\text { Vulcanite. }\end{array}$ & $\begin{array}{l}\text { R. 3. Coloradase. } \\
\text { Granite. } \\
\text { Granodiorite. } \\
\text { Quartz-diorite. } \\
\text { Banatite. } \\
\text { Diorite. } \\
\text { Dacite. } \\
\text { Andesite. } \\
\text { Trachyte. }\end{array}$ & $\begin{array}{l}\text { 1R. 3. } \\
\text { S.enite. } \\
\text { Diubrse-porphy- } \\
\text { rite. } \\
\text { Andesite. } \\
\text { 1. 4. Labrador- } \\
\text { Ano. } \\
\text { Anorthosite. } \\
\text { Ano. Canadase. } \\
\text { Anorthosite. }\end{array}$ & - & . & 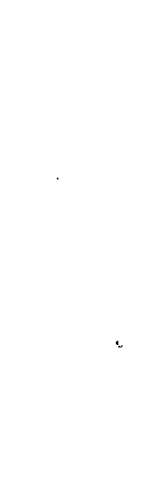 & \\
\hline
\end{tabular}


CLASS II. DOSALANE.

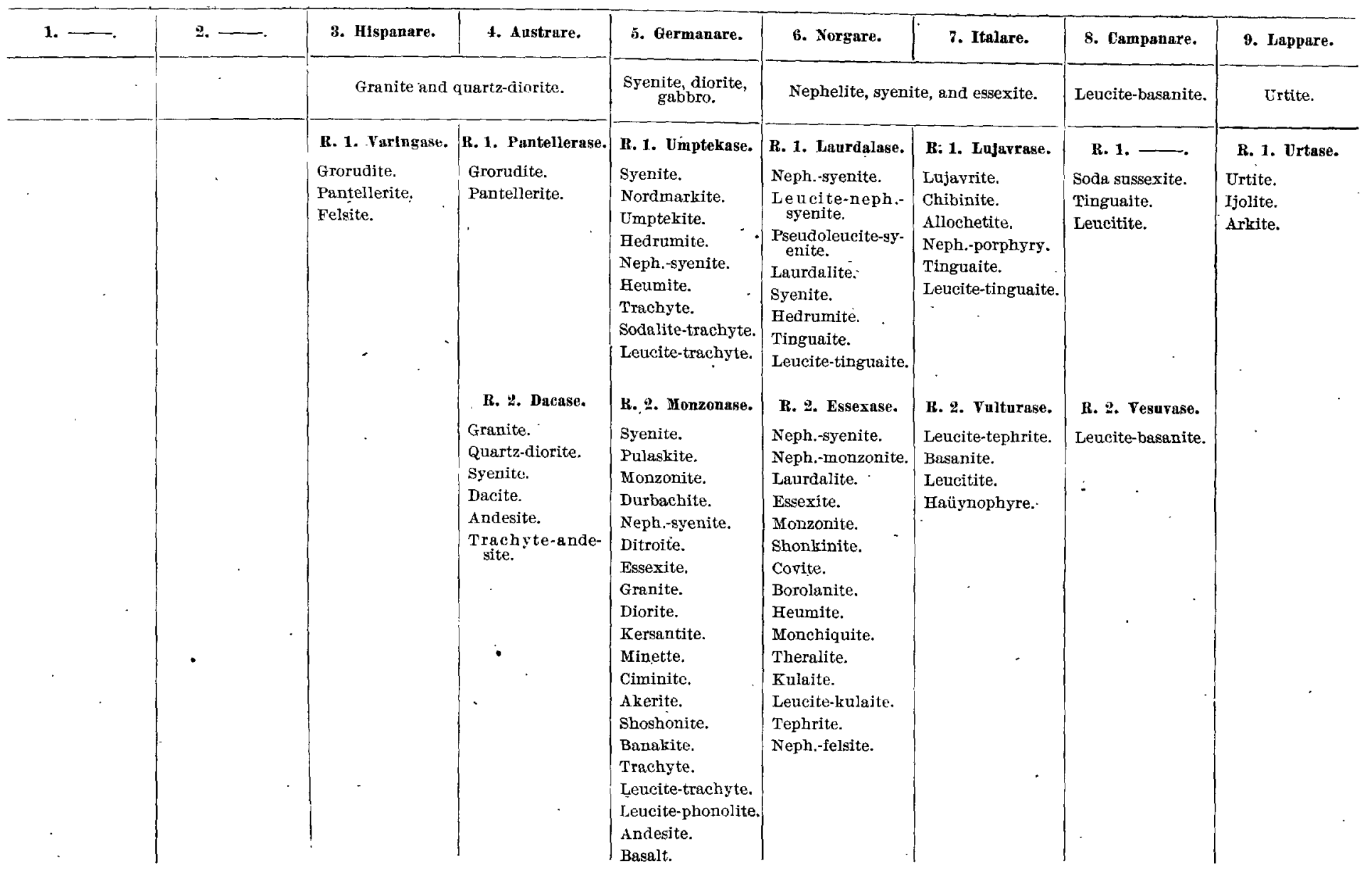


CLASS II. DOSALANEE-Continued.

\begin{tabular}{|c|c|c|c|c|c|c|c|c|}
\hline 1. - . & 9. - & 3. Hispanare. & 4. Austrare. & 5. Germunare. & 6. Norgare. & 7. Italare. & \multirow{2}{*}{$\frac{\text { 8. Campanare. }}{\text { Leucite-basinite. }}$} & 9. Lappare. \\
\hline & & \multicolumn{2}{|c|}{ Granite and quartz-diorite. } & $\begin{array}{c}\text { Syenite, diorite, } \\
\text { gabbro. }\end{array}$ & \multicolumn{2}{|c|}{ Nephelite, syenite, and essexite. } & & Urtite. \\
\hline & . & R. 4. - & $\begin{array}{l}\text { 1. 3. Tomilase. } \\
\text { Granite. } \\
\text { Quartz-monzon- } \\
\text { ite. } \\
\text { Granodiorite. } \\
\text { Quartz-diorite. } \\
\text { Tonalite. } \\
\text { Quartz-norite. } \\
\text { Syenite. } \\
\text { Diorite. } \\
\text { Dacite. } \\
\text { Latite. } \\
\text { Andesite. } \\
\text { Trachyte. } \\
\text { Dolerite. } \\
\\
\\
\\
\text { R. 4. Bandase. } \\
\text { Tonalite. } \\
\text { Diorite. } \\
\text { And_site. } \\
\text { Quartz-basalt. } \\
\quad \\
\text { R. 5. } \\
\text { Diorite. }\end{array}$ & $\begin{array}{l}\text { R. 3. And lase. } \\
\text { Syenite. } \\
\text { Monzonite. } \\
\text { Diorite. } \\
\text { Gabbro. } \\
\text { Norite. } \\
\text { Essexite. } \\
\text { Beerbachite. } \\
\text { Mondhaldeite. } \\
\text { Kersantite. } \\
\text { Andesite. } \\
\text { Latite. } \\
\text { Vulsinite. } \\
\text { Ciminite. } \\
\text { Shoshonite } \\
\text { Banakite. } \\
\text { Quartz-basalt. } \\
\text { Basalt. } \\
\text { Dolerite. } \\
\text { Olivine-diabase. } \\
\text { Leucite-mon chi- } \\
\text { quite. } \\
\text { Neph.-tephrite. } \\
\text { R. 4. Hessase. } \\
\text { Diorite. } \\
\text { Monzonite. } \\
\text { Anortbosite. } \\
\text { Gabbro. } \\
\text { Corsite. } \\
\text { Andesite. } \\
\text { Busalt. } \\
\text { R. 5. Corsase. } \\
\text { Diorite. } \\
\text { Olivine-gabbro. }\end{array}$ & $\begin{array}{l}\text { 1. :. Salemake. } \\
\text { Diorite. } \\
\text { Gabbro. } \\
\text { Essexite. } \\
\text { Basalt. }\end{array}$ & $\begin{array}{l}\text { R. 3. }- \\
\text { Leucite-tephrite. }\end{array}$ & 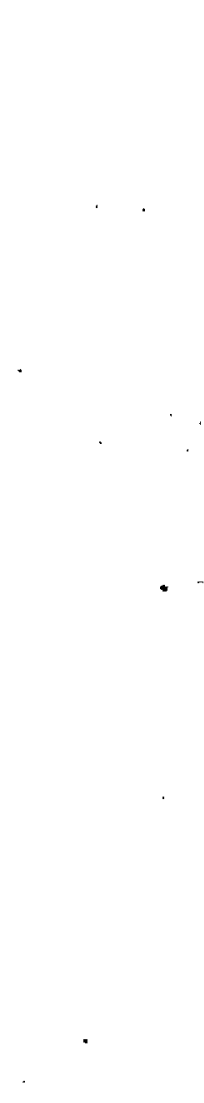 & $:$ \\
\hline
\end{tabular}


CLASS III. SALFEMANE.

\begin{tabular}{|c|c|c|c|c|c|c|c|c|}
\hline \multirow[t]{2}{*}{ 1. - - } & 2. - - & 3. Atlantare. & t. Vualiare. & 5. 'Gullare. & 6. Portugare. & 7. Kamerunare. & s. Böhemare. & 9. Finnare. \\
\hline & & & \multicolumn{2}{|c|}{ Diorite and gabbro. } & \multicolumn{4}{|c|}{ Lamprophyres and leucite-nephelite lavas. } \\
\hline . & & $\begin{array}{l}\text { R. 1. Rockall.sir. } \\
\text { Kockallite. } \\
\text { Pantellerite. }\end{array}$ & $\begin{array}{l}. \\
\\
\text { R. 3. Vanluse. } \\
\text { Quartz-gabbro. } \\
\text { Olivine-diabase. } \\
\text { Basalt. } \\
. \\
.\end{array}$ & \begin{tabular}{|l} 
R. 1. Orendase. \\
Orendite. \\
Wyomingite. \\
. \\
R. 2. kulauase: \\
Shonkinite. \\
syenite-lam p ro- \\
phyre. \\
Micici-picrophyre. \\
Absarokite. \\
Basalt. \\
Augitite. \\
\\
\\
\\
R. 3. Camptonase. \\
Kentallenite. \\
Olivine - monzon- \\
ite. \\
Diorite. \\
Gabbro. \\
Essexite. \\
Camptonite. \\
Absarokite. \\
Leucite-busanite. \\
Neph.-bisalt. \\
Quartz-basalt.
\end{tabular} & 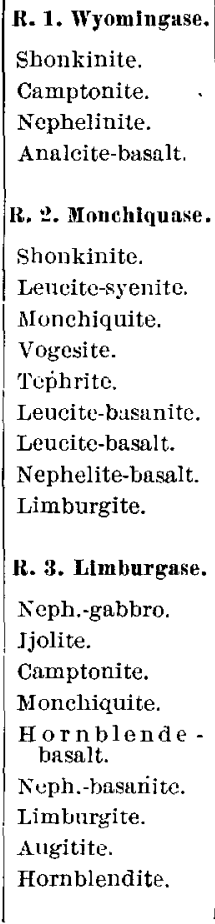 & $\begin{array}{l}\text { R. 1. Malignase. } \\
\text { Malignite. } \\
\text { Therilite. } \\
\text { Ijolite-porphyry. } \\
\\
\text { R. g. Kamerunlase. } \\
\text { Heumite. } \\
\text { Theralite. } \\
\text { Monchiquite. } \\
\text { Furrisite. } \\
\text { Leucite-basalt. } \\
\text { Leucite-nephelin- } \\
\text { ite. } \\
\text { Haüynophyre. } \\
\text { Basalt. } \\
\text { R. B. EtIndase. } \\
\text { Camptonite. } \\
\text { Neph.-basalt. }\end{array}$ & $\begin{array}{l}\text { R. 1. Chotuse. } \\
\text { Leucitite. } \\
\text { R. 2. Albanasse. } \\
\text { Ijolite. } \\
\text { Nephelinite. } \\
\text { Leucitite. } \\
\text { Theralite. } \\
\text { Leueite-tephrite. } \\
\text { Limburgite. }\end{array}$ & $\begin{array}{l}\text { R. 1. Ijolase. } \\
\text { Madupite. } \\
\text { Ijolite. } \\
\text { Nephelite-por- } \\
\text { phyry. } \\
\text { Neph.-basalt. }\end{array}$ \\
\hline
\end{tabular}


CLASS III. SALFEMANE-Continued.

\begin{tabular}{|c|c|c|c|c|c|c|c|c|}
\hline \multirow[t]{3}{*}{ 1. - - } & \multirow[t]{2}{*}{ 2. - } & \multirow[t]{2}{*}{ 3. Atliutare. } & 4. Vaulitre. & 5. Gallare. & 6. Portugare. & 7. Kamerumare. & 8. Bohémare. & 9. Finnare. \\
\hline & & & \multicolumn{2}{|c|}{ Diorite and gubbro. } & \multicolumn{4}{|c|}{ Lamprophyres and leucite-nephelite lavas. } \\
\hline & . & & \begin{tabular}{|l|}
\multicolumn{1}{|c|}{ R. 4. } \\
Diorite. \\
Melaphyre. \\
\\
R. 5. - \\
Olivine-g a b b ro- \\
diabase.
\end{tabular} & $\begin{array}{l}\text { R. 4. Auvergnase. } \\
\text { Gabbro. } \\
\text { Norite. } \\
\text { Dolerite. } \\
\text { Ariegite. } \\
\text { Fourchite. } \\
\text { Monchiquite. } \\
\text { Basalt. } \\
\text { R. 5. Kedahekase. } \\
\text { Gabbro. } \\
\text { Norite. } \\
\text { Kedabekite. } \\
\text { Ariegite. }\end{array}$ & $\begin{array}{r}\text { R. 4. } \\
\text { Gabbro. }\end{array}$ & - & $\because$ & : \\
\hline
\end{tabular}


CLASS IV. DOFEMANE.

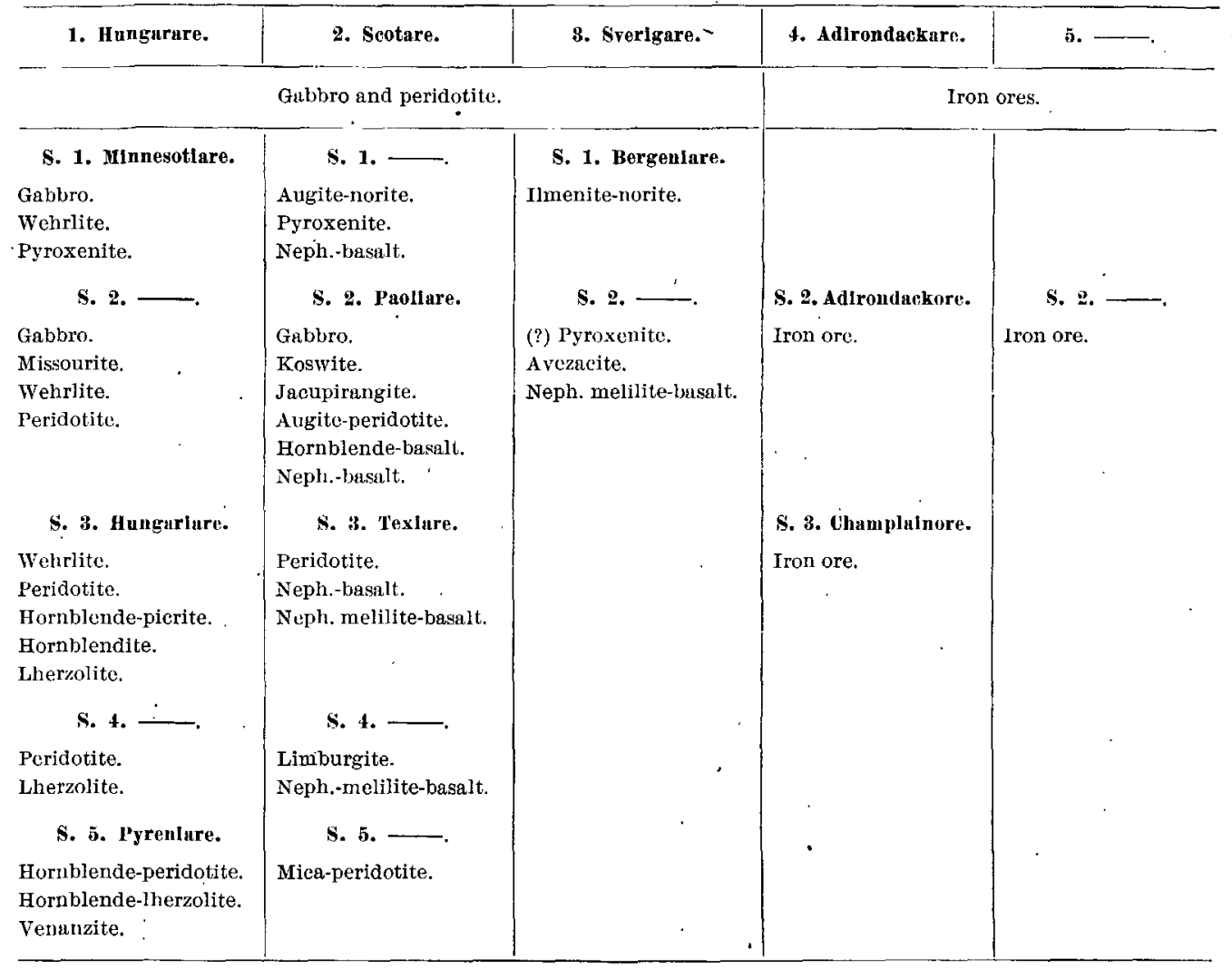

CLASS V. PERFEMANE.

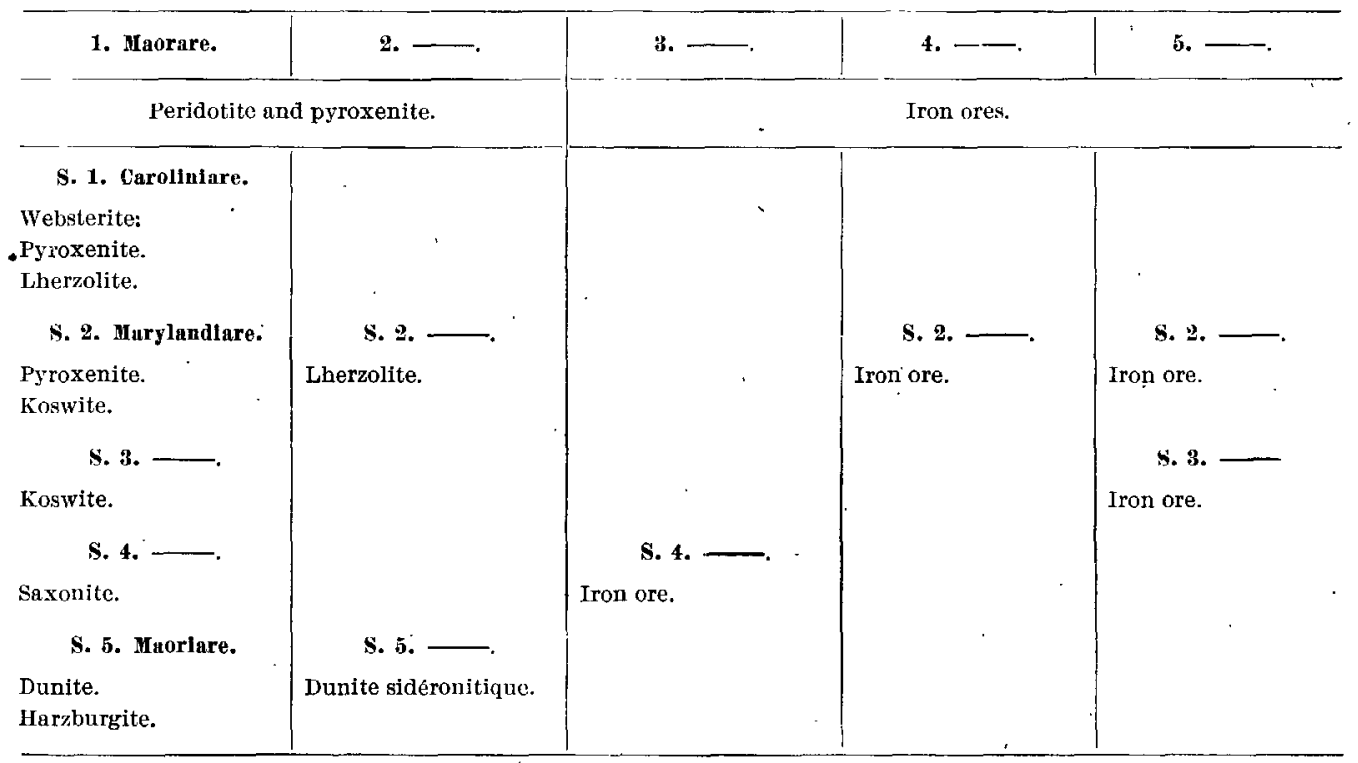


Approaching the subject from the standpoint of the qualitative, mineralogical system we are interested in the distribution of rocks in the quantitative system in the first instance according to their content of quartz, feldspar, feldspathoid, or ferromagnesian minerals. We may consider the distribution of, first, rocks containing a notable amount of quartz and their equivalent lava forms; second, rocks characterized by feldspar without notable amounts of quartz or feldspathoids; third, rocks containing notable amounts of feldspathoids; fourth, rocks composed chiefly of ferromagnesian minerals.

In general, it may be said, that the quartzose igneous rocks fall almost without exception in Classes I, II, and. III, and those in Class III are comparatively rare, so that most quartzose rocks belong to Classes I and II, and those with notable amount of quartz $\left(\frac{\mathrm{Q}}{\mathrm{F}}>\frac{1}{7}\right)$ are in orders 4 and 3 , very few occurring in orders 2 and 1 , and these only in Class $I$.

Nearly all rocks known as granite, granodiorite, quartz-monzonite, quartz-. diorite, and the rare quartz-gabbros, together with their aphanitic and glassy equivalents, belong to order 4, Classes I and II, in most part, and to some extent to order 3 in Class I, very few being found in this order of Class II. 'The most quartzose rocks belong to Class $I$.

Of the minerals associated with quartz the most abundant and frequent are feldspars, usually preponderant, and these are distinguished in the qualitative system as alkalic, alkalicalcic, and calcic. Since it is generally true that as magmas. are richer in magnesium and iron they also contain more calcium, it follows that rocks richer in ferromagnesian minerals usually carry more calcic feldspars than rocks poor in those minerals. Consequently the greater number of quartzose rocks with alkalie feldspars occur in Class I, and the greater number with culcic feldspars are in Class II; and those with the most calcic feldspar. should occur in Class III. To this, of course, there are notable exceptions, especially where the femic mineral is not ferromagnesian but alkalic, as acmite, in rockallose.

Most granites are found in Class I, and most of these are in order 4, with dominant feldspars; fewer in order 3 . Most of these persalanes are peralkalic and domalkalic, rangs 1 and 2 . But in order 4 a considerable number are alkalicalcic, rang 3. The more alkalic rocks are commonly sodipotassic, while the alkalicalcic ones are mostly dosodic; that. is, with increase of lime there is a diminution of potash.

These quartz-bearing persalanes include rocks that have been named aplite, granite, granitite, granodiorite, quartz-monzonite, quartz-diorite, the phanerocrystalline porphyries of these, and quartz-porpbyry, rhyolite, dacite, and andesite, together with those of special varieties--alaskite, alsbachite, tordrillite, comendite, 
paisanite, toscanite, and others. Some rocks that fall within the limits of these divisions of the quantitative system bear names occurring mostly in other divisions, such as syenite, nordmarkite, diorite, andesite. These are in part intermediate rocks deserving double magma names; in part, those, like some andesites, whose mineral composition is not completely determinable, and in which the quartz content is obscure or uncrystallized.

A much smaller number of granites belong to Class II, dosalanes. The most alkalic members of this class, pantellerite and grorudite, are comparatively rich in ferric oxide.

Most of the quaric rocks of Class II are alkalicalcic and dosodic. The greater number of them bear the names quartz-diorite, diorite, and andesite.

The quaric rocks $i$. Class III are low in quartz, except in the rare varieties of pantellerite and rockallite. But in the other rocks, also rare, quartz occurs in small amount with alkalicalcic feldspars and calcic feldspars. One of these rocks is quartz-gabbro, another a diorite, and several others olivine-bearing rocks, whose chemical analyses are such that they fall in order 4 of this class.

Rocks characterized by feldspars without notable amounts of quartz or feldspathoids occur in perfelic order 5 of Classes I, II, and III.

Among the perfelic persalanes, Class I, the more alkalic rocks are commonest (syenites), alkalicalcic ones are rare, while docalcic ones are well known (anorthosites). As to the character of the alkalies, soda preponderates in most cases, dosodic rocks being more abundant than sodipotassic ones, which, however, are common.

The names borne by these rocks arc: Syenite, nordmarkite, pulaskite, laurvikite, bostonite, trachyte rulsinite, andesite, keratophyre, and others. The most sodic rocks have been called albitite; the most calcic, anorthosite. Some of them have been called nephelite-syenite, phonolite, leucite-trachyte, and other names.

The perfelic dosalanes, Class II, are more common than the extremely feldspathic persalanes, and the alkalicalcic ones are more numerous than the domalkalic or docalcic forms, but these are also well represented. Most of these rocks are dosodic, although many are sodipotassic.

They appear under the names of syenite, umptekite, monzonite, diorite, gabbro, norite, trachyte, ciminite, akerite, shoshonite, andesite, basalt, and others. They also include some rocks called granite, nephelite-syenite, leucite-trachyte, and kersantite.

The perfelic salfemanes, Class III, are alkalicalcic and docalcic, and most of them are dosodic. They embrace gabbro, norite, ariegite, kedabekite, camptonite, dolerite, and basalt. The more potassic and sodipotassic rocks of this class and order have been called kentallenite, absarokite, and lamprophyre. A 
few peralkalic rocks belonging here occur at Leucite Hills, Wyoming-orendite, wyomingite.

The rocks characterized by notable amounts of feldspathoids or lenads-leucite, nephelite, sodalite-with the exception of a few that belong in Class IV, fall within the lenic orders $6,7,8,9$ of Classes I, II, and III.

Lenic persalanes, Class I, are most abundant in order 6, with dominant feldspar, but they are known in each of the more lenic orders. They are mostly peralkalic and dosodic, some being sodipotassic. They have been named nephelitesyenite, foyaite, ditroite, miascite, phonolite, and tinguaite. Very few contain leucite.

The same is true for the lenic dosalanes; Class II. Most of them occur in lendofelic order 6 , but the other lenic orders are represented.

In order 6 they are mostly peralkalic and domalkalic, and the majority are dosodic. They include nephelite-syenite, laurdalite, essexite, tinguaite, theralite, and others, besides some rocks called syenite, monzonite, diorite, and gàbbro:

Rocks of the remaining orders are peralkalic and dominantly sodic. A few rocks in order 7 are sodipotassic.

Order 7 contains rocks called lujavrite, tinguaite, tephrite, leucitite, hauynophyre, and othẻrs.

Rocks of orders 8 and 9 are rare. The first embrace leucite-basanite, leucitite, and tinguaite. The ninth order is represented by urtite, an ijolite rich in nephelite, and sodipotassic arkite.

Lenic salfemanes, Class III, are about as numerous as lenic rocks in Classes I and II. They are more uniformly distributed through orders 6,7 , and 8 . Perlenic rocks, order 9 , are rare.

In order 6 there are more alkalicalcic rocks than in the corresponding order of Class II. Most of the rocks are sodipotassic and dosodic. The rocks have been called shonkinite, leucite-syenite, leucite-monchiquite, monchiquite, limburgite, basanite, and others.

In order 7 the rocks are dominantly alkalic and dosodic. They embrace malignite, theralite, nephelite-basalt, nephelinite, and others.

Order 8 is similar to order 7 . The rocks are rarer and a few are dopotassic. They are theralite, ijolite, nephelinite, leucitite, and others.

The rocks of order 9 are peralkalic, and include dopotassic madupite of . Leucite Hills, Wyoming; and dosodic ijolite, nephelite-porphyry, and nephelitebasalt.

Rocks with preponderant femic constituents, the dofemanes, Class IV, and perfemanes, Class V, are distinguished from one another by the presence in the former of a notable but subordinate amount of salic, feldspathic minerals and 
by their absence from the perfemanes. The dofemanes, then, contain either nota ble subordinate amounts of feldspar, leucite, nephelite, sodalite, or of aluminous ferromagnesian, alferric minerals.

In Class IV the five orders, based on the proportions of normative pyroxene and olivine, compared with magnetite, ilmenite, and other nonsilicates, are all represented. Orders 1 and 2 are most common. In each of these the more pyroxenic sections are better-represented than the more olivinic. The rocks invariably belong to the permirlic rang, 1; that is to say, the femic minerals contain little or no alkali. In most cases ferrous oxide and magnesia preponderate over lime, and in the majority of rocks magnesia dominates over ferrous oxide.

In perpolic order 1 the rocks ure known in the qualitative system as gabbro, olivine-gabbro, norite, koswite, peridotite, pyroxenite, jacupirangite, nephelitebasalt, nephelite-melilite-basalt, and limburgite.

In polmitic order 3 , with ncarly equal silicate and nonsilicate minerals, the few rocks as yet described have been called ilmenite-norite, pyroxenite, avezacite, and nephelite-melilite-basalt.

Orders $t$ and 5 , with preponderant magnetite and ilmenite, embrace certain feldspathic iron ores from Sweden, Canada, and New York State.

Rocks of Class $\mathrm{V}$ are comparatively rare; they have little or no feldspathic minerals or aluminous ferromagnesian minerals. Most rocks belong to perpolic order 1, and range from wholly pyroxenic to wholly olivinic kinds. A very fow rocks fall within each of the other orders.

In order 1 the rocks bave been called pyroxenite, websterite, koswite, peridotite, saxonite, harzburgite, and dunite.

In order 2, with notable, subordinate, nonsilicate minerals, occur a lherzolite, and dunite rich in iron ore.

In the other orders, 3,4 , and 5 , the rocks are titaniferous iron ores, with variable amounts of ferromagnesian silicates. In one instance the rock consists of magnetite and spinel 


\section{N D EX}

A.

Pnge.

Abbreviations, list of..

Absarokite.

(1) 49,86

Acmite-trachyte ..................................... 31, 34

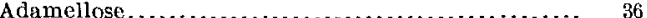

Adirondackare .............................. 58-59,88

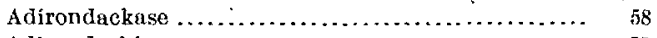

Adirondackiase ............................... 58

Adirondackore $\ldots \ldots \ldots \ldots \ldots \ldots \ldots \ldots \ldots \ldots \ldots \ldots, 58,88$

Aegirite-granite .

Africa, rocks from ....................... 44, 47,52,53,59

A kerite ...................................... 28,40,84

Akerose $\ldots \ldots \ldots \ldots \ldots \ldots \ldots \ldots \ldots \ldots \ldots \ldots \ldots \ldots,{ }_{40}$

Alaska, rocks from ............. 23, 24, 26,28,29, $38,41,42,67$

Alaskase ..................................... 23-24, 82

Alaskite ................................. 23, 26, 28,82

Alaskite-porphyry ............................. $\quad 29$

Alaskose ......................................... 23

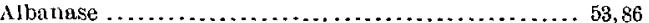

Albanose ..................................... 53

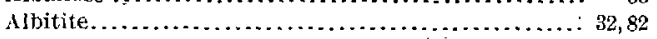

Allochetite ................................. 45,84

Alsace, rocks from ................ 24, 30, 36, 42, 43, 51,61,68.

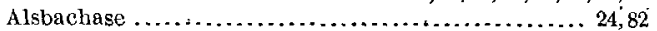

Alsbachite ................................... 24,82

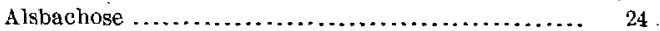

Amadorose

America, North, average rock from

Amiatose

Amphibole-malignite.

Analcite-basalt

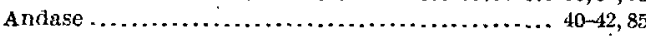

Andes:te $\ldots \ldots \ldots$ 29, 30, 32, 33, 35, 36, 37, 38, 40,41, 42, 82, 83, 84, 85

phonolitic

Andesite-porphy ry ................................. 29,37

-Andesite-basalt.................................

Andesite-perlite ............................... 30

Andesitic glass

Andose

Anorthosite .................................. $33,42,48,83,85$

Aplite ..... .

$33,42,48,83,85$
$23,24,26,28,82$

Aplitic granite.

A plitic granophyre............................... 23,26

Argeinose.

Ariegite

A rizona, rocks from .......................... 40,49

A rkansas, rocks from . .......... 31, 32, 34, 35, 43, 46, 53, 56, 68

Arkänsos

46
46,84

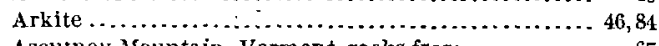

Ascutney Mountain, Vermont, rocks from ......... 67

Asia, rocks from .............................. 56

Asia Minor, rocks from.......................... $30,37,44$

Atlantare

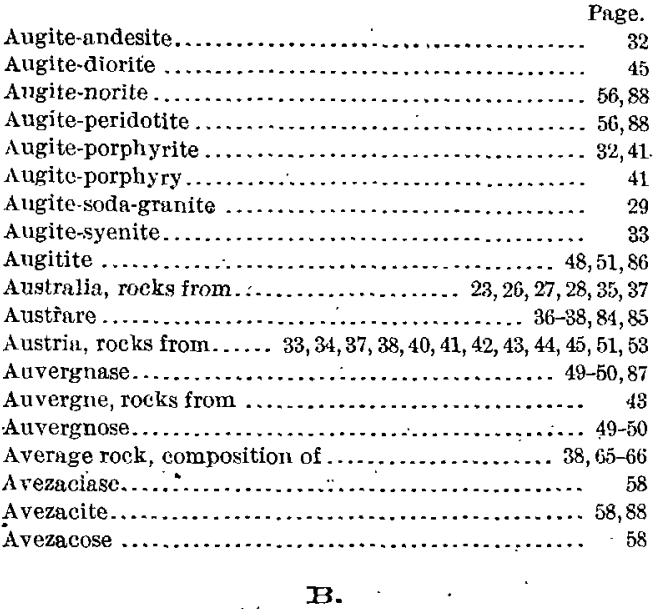

Baden, rocks from $\ldots \ldots \ldots \ldots \ldots .89,40,41,50,51,52,56,57,58$

Baltinorase................................... 60

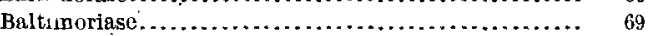

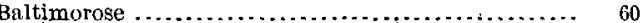

Banakite .......................... 40, $41,84,85$

Banatite................................. 30,83

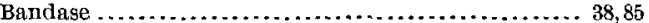

Bandose ...................................... 38

Basalt...........40,41, 42, 43, 45, 47, 48, 49, 50, 52, 84, 85, 86, 87

Basanite................................ 45,46,84 Sce also Leucite, Nephelite-basanite.

Bavarí, rocks from $. \ldots \ldots \ldots \ldots \ldots \ldots \ldots \ldots \ldots \ldots \ldots 2,54$

Bearpaw Mountains, Montant, rocks from.......... 27,40

Becke, F., diugrams used by, description of .......... 11

Beemerose.. ................................. 34

Beerbachite $\ldots \ldots \ldots \ldots \ldots \ldots \ldots \ldots \ldots \ldots \ldots \ldots \ldots \ldots, 42,85$

Beërbachose ............................... ${ }_{42}$

Beleherose................................. 54

Belgare $. \ldots \ldots \ldots \ldots \ldots \ldots \ldots \ldots \ldots \ldots \ldots \ldots \ldots \ldots \ldots . .62-83$

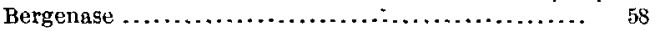

Bergeniare................................ 58,88

Bergeniase. ................................. 58

Bergenose $\ldots \ldots \ldots \ldots \ldots \ldots \ldots \ldots \ldots \ldots \ldots \ldots \ldots \ldots \ldots \ldots, \quad 58$

Bluck Forest, rocks from . ................... $27 \%$

Bogoslof Island, Alaska, rocks from............ 41; 42

Bohemare ........................... 53,86-87

Bohemiu, rocks from............... 24,41, 42, 44, 48,51,58

Borolanite............................... 44,84

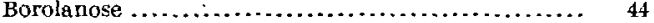

Bostonite ................................ 31, 39, 82

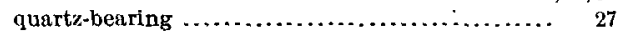

Brandbergiase............................... 56 


\section{INDEX.}

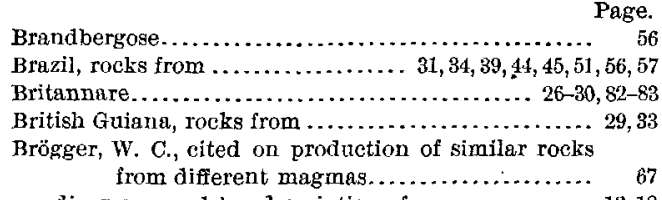

dingrams used by, description of

\section{C.}

California, rocks' from................. 23, 24, 25, 26, 27, $28,29,30,31,37,38,39,41,42,43,49,50,55,60,67,68$

Campanare $\begin{array}{r}46,84-85 \\ \hline\end{array}$

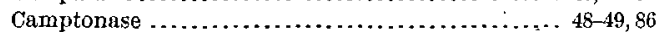

Camptonite........................ 42, 49,50,51,53,86

Camptonitic tingunite.

49

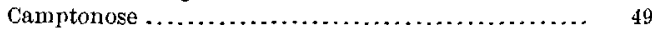

Cantada, rocks from........ 31, $32,33,34,40,42,44,52,58,59,62$

Canadaro ........................................ $31-33,82-83$

Canadase .................................... 33,83

Cape Colony, rocks from.

Cape Verde, rocks from................. 52, 53

Caroliniare ............................. $59-60,88$

Casselase ................................. 57

Casseliase.................................... 57

Casselose................................... 57

Castle Mountains, rocks from ................. 40,48

Cecilose....................................... 60

Celebes, rocks from ................... 31, 32, 34, 40,44

Champlainase .................................. $\quad 59$

Champlainisse .............................. 59

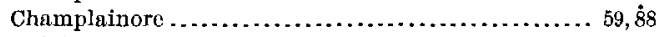

Chibinite .................................. 45,84

Chile, rocks from ........................ 28, 39, 42,51

Chotase ................................. 53,86

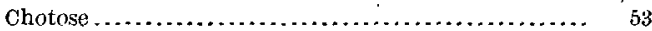

Christiania region, Norway, rocks from ............ 67-68

Ciminite .........................

Ciminose .................................. 39

Clarke, F. W., cited on composition of average rock... 65,66

Classification of igneous rocks, muthods of :........ $68-70$

Colombia, rocks from................ $30,36,37,40,41$

Coloradase. .

Colorado, rocks frorn................ 23, 26, 27, 28, 29, 30, 31,$33 ; 34,37,38,39,40,41,42,43,44,45,48,49,51,55,67,68$

Coloradose.

Columbare ...................................... 23-25, 82-83

Columbretes Islands, rocks from ............. 33, 34, 40

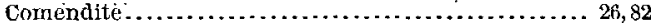

Connecticut, rocks from.................. 26, 28, $33,49,67$

Cookose................................... 54

Correlation of igneous rocks, tables showing......... 81-92

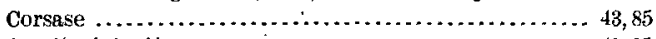

Corsite................................. 43, 85

cortlandtase................................. 55

Cortlund tiase................................. 55

Cortlandtose .................................. 55

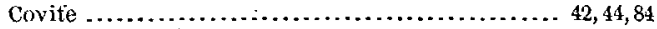

Covose ......................................... 53

Crazy Mountains, Montana, rocks from ............ 31 ,

$33,39,41,42,52,56,67$

Cripple Creek, Colorado, roeks from............. 67

Cross, Whitmun, diagrams used by; reference to ...... . .11

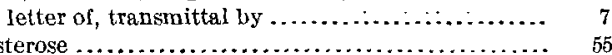

Custerose

D.

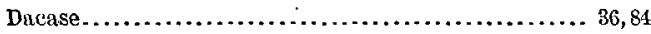

Dacite $\ldots \ldots \ldots \ldots \ldots \ldots \ldots \ldots \ldots \ldots .28,29,30,36,37,82,83,84,85$ secretion in.
Puge.

Dacite-porphyry $\ldots \ldots \ldots \ldots \ldots \ldots \ldots \ldots \ldots \ldots \ldots \ldots \ldots \ldots \ldots \ldots \ldots \ldots \ldots, 29,30$

Dacose..................................... 36

Dakyns, J. R., and Teall, J. J. H., diagrams used by - reference to........................ 10-11 Dargase ................................... 82

Dargose ..................................... 82

Dellenite....................................... 27,82

Dellenose .................................... ${ }_{27}$

Diabrse $\ldots \ldots \ldots \ldots \ldots \ldots \ldots \ldots \ldots \ldots \ldots \ldots \ldots \ldots \ldots \ldots 42,49,50$

Diabase-pitchstone .......................... 49

Diabase-porphyrite ......................... 33,83

Diabase-porphyry ............................. $\quad 30$

Diubase-syenite-porphyry ..................... $\quad 42$

Diagrams, classification of......................... $70-81$

classifiention of, Class I....................... $71-76$

Class'II ............................... $76-77$

Class III . . . . . . . . . . . . . . . . . . . . . . . $77-79$

Classes IV and V ....................... $79-81$

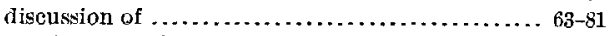

previous use of $\ldots \ldots \ldots \ldots \ldots \ldots \ldots \ldots \ldots \ldots \ldots \ldots, 10-16$

Dike rock ................................... 57

Diorite................................. 29 ,

$30,33,37,38,40,41,42,43,45,47,49,82,83,84,85,86,87$

Diorite-porphyrite ........................... 42

Diorite-porphyry .......................... 40

District of Columbia, rocks from ............. 38,47

Ditroite............................. $34,40,82,84$

Dofemnne .............................. 54-59,88

Dolerite $\ldots \ldots \ldots \ldots \ldots \ldots \ldots \ldots \ldots \ldots \ldots . \ldots \ldots, 41,42,43,49,85,87$

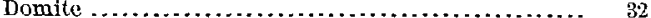

Dosalane .................................... $35-47$

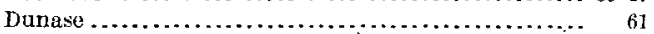

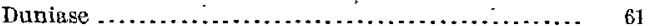

Dunite................................... 61,88

Dunite sidéronitique $\ldots \ldots \ldots \ldots \ldots \ldots \ldots \ldots \ldots \ldots \ldots .61,88$

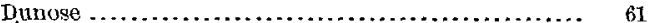

Durbachite $\ldots \ldots \ldots \ldots \ldots \ldots \ldots \ldots \ldots \ldots \ldots \ldots \ldots \ldots . . \ldots, 89,84$

F.

Eleolite-mica-syenite........................ 56

Elsass. See Alsace. .

England, rocks from $\ldots \ldots \ldots \ldots \ldots \ldots \ldots \ldots \ldots \ldots \ldots .28$

Essexase .................................... 44,84

Essexite ....................... $40,42,44,45,49,84,85,86$

Essexose ................................... 44

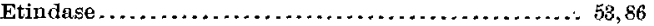

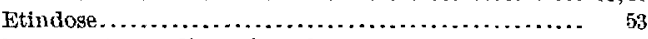

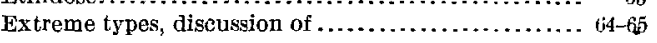

H.

Farrisite ............................... 52,86

Feldspar-porphyry ........................... . 29

Felsite ................................... 35,84

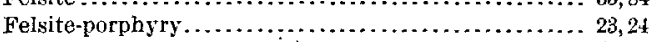

Ferdinandea, rocks from $\ldots \ldots \ldots \ldots \ldots \ldots \ldots \ldots \ldots \ldots+42,68$

Fergusose................................ 43

Fichtelgebirge, rock from ................... 23

Finland, rocks from.. $24,26,31,33,34,35,39,45,46,47,52 ; 54,57$

Finnare............................ 58-54,86-87

Fourchite.................................. 50,87

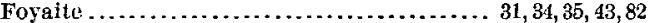

France, rocks from............... $32,34,40,43,50,55,58$

\section{G.}

Gabbro ................. 43, 45, 49,50,54,84, 85, 86, 87,88

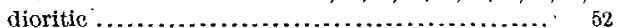
Gabbro-diorite.................................... 4], 43 Gabbro-porphyry ............................. 49 
Page.

Gallare

Garnet-pyroxene-malignite

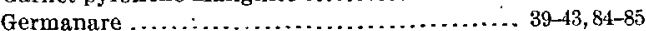
Germany, rocks from.... 23, 26, 31, 34, 45, 49,50,51,53, $54,56,57$ Glass, andesitic.

Graham Island, rocks from ...................... Granite ....... 23, 24, 25, 26, 27, 28, 29, 30, 37, 38, 39, 82, 83, 84, 85 graphic ..... Granite-syenite-porphyry.................... 27,28 Granite-porphyry $\ldots \ldots \ldots \ldots \ldots \ldots \ldots \ldots \ldots 2,26,27,28,29,36$ Granitite................ 23, 24, 20, 26, 27, 28,29, 30, 36, 37 Granodiorite........................... 29, 30, 82,83,85 Granodiorite-aplite .......................... 28

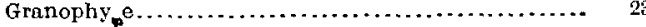

Graphic granite............................... Great Britain, rorks from............. $23,28,38,41,44,49$ Greenland, rocks from ......................... 50

Grorudite. Grorudose $27,35,36,82,84$ F.

Hackman, V., diagrams used by, reference to........ 13 Hanover, rocks from .......................... 49 Harker, A., cited on composition of the arerage rock.. 65-66 diagrams used by, reference to ...

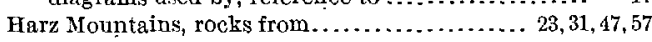

Harzburgite . .............................. 61, 88

Harzose ........................................ $\quad 37$

Haüynophyre....................... 46, 52, 84, 86

Hedrumite............................... 39,44,84

Heronite .................................... 34, 82

Herzegovina, rocks from ..................... 33

Hessase $\ldots \ldots \ldots . \ldots \ldots \ldots \ldots \ldots \ldots \ldots \ldots \ldots \ldots \ldots .42-43,85$

Hessose $-42-43$

Hesse, rocks from ................... 25, 42, 45, 51, 53

Heumite ......................... 39, 44, 52,84,86

Highwood Mcountains, Montana, rocks from ......... 31 $36,40,41,43,51,52$

Highwoodose ................................ 39

Hispamare .............................. 35-36,84-85

Hobbs, W. H., diagrams used by, description of ...... 13

Hornblende-basalt $\ldots \ldots \ldots \ldots \ldots \ldots \ldots \ldots \ldots \ldots \ldots 51,56,86,88$

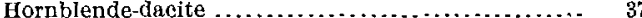

Hornblende-gabbro........................ 45,52

Hornblende-1herzolite ......................... 55,88

Hornblende-monehiquite...................... 50

Hornblende-peridotite....................... 55, 88

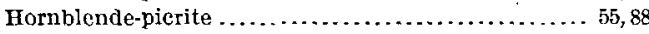

Hornblende-porphyrite.

Hornblende-rogesite....................... 51

Hornblendite $\ldots \ldots \ldots \ldots \ldots \ldots \ldots \ldots \ldots \ldots \ldots \ldots .51,5 E, 86,88$

Hungary, rock from . . ................... 24,30,34,37

Hungarare............................... 54-56,88

Hungariare ............................ 55, 88

Hypersthene-gabbro.

I.

Idaho, rocks from...

Iddings, J. P., diagrams employed in 1890 and $1898 \mathrm{by}$ description of ................... 10, 13-14

Iivan rose $\ldots \ldots \ldots \ldots \ldots \ldots \ldots \ldots \ldots \ldots \ldots \ldots \ldots \ldots \ldots \ldots, 54$

Ijolase $\ldots \ldots \ldots \ldots \ldots \ldots \ldots \ldots \ldots \ldots \ldots \ldots \ldots \ldots \ldots \ldots \ldots \ldots \ldots \ldots, 54,86$

ljolite $\ldots \ldots \ldots \ldots \ldots \ldots \ldots \ldots \ldots \ldots \ldots \ldots 47,51,53,54,81,86$

Ijolite-porphyry ......................... 52,86

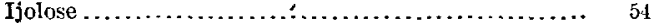

Ilmenite-norite ............................ $58-88$

Ilmenose . . . . . . . . . . . . . . . . . . . . . . . . .

India, rock from.
Ireland, rock from $\ldots \ldots \ldots \ldots \ldots \ldots \ldots \ldots \ldots \ldots \ldots \ldots{ }_{23}$

Iron Mountain, Missouri, rocks from .............. 67

Iron ore $\ldots \ldots \ldots \ldots \ldots \ldots \ldots \ldots \ldots \ldots \ldots \ldots . \ldots \ldots, 59,61,62,88$ Ischia, Italy, rocks from .................... 40,67 Italy, rocks from ........................... 25, 26, $27,28,29,31,32,34,35,39,40,41,42,43,44$ $45,46,47,48,49,50,52,53,55,56,58,59,67$

Italare $45-46,84-85$

J.

Jacupirangite.

Janeirose $\ldots \ldots \ldots \ldots \ldots \ldots \ldots \ldots \ldots \ldots \ldots \ldots \ldots \ldots \ldots \ldots,{ }_{45}$

Japan, rocks from .............................. 38

Judd, J. W., diagrum employed by, reference to...... 10

Judithose . .................................. 43

\section{K.}

Kaiserstuhl, rocks from $. . . \ldots \ldots \ldots \ldots \ldots \ldots \ldots \ldots . .41$

Kallerudose.................................. 27

Kalteniase ......................................... 57

Kaltenose................................. 57

Kamerunare ......................... 52-53,86-87

Kamerunase.................................. 52,86

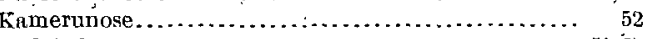

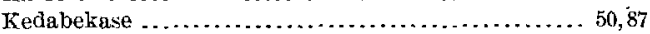

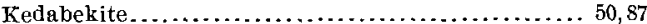

Kentallenile............................... 49, 86

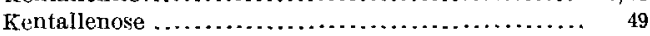

Kentucky, rocks from.......................... 39

Keratophyre ........................... $31,83,82$

Kersantite............................. 39,42,84,85

Kilauase.................................. 48,86

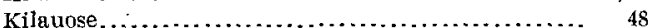

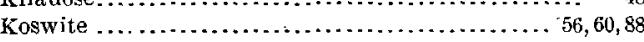

Koswose .................................... 60

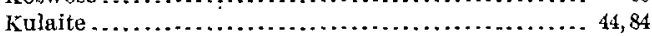

I.

Labrador, rocks from. .........................

Labradorase $. . \ldots \ldots \ldots \ldots \ldots \ldots \ldots \ldots \ldots \ldots \ldots \ldots \ldots . ., 33,83$

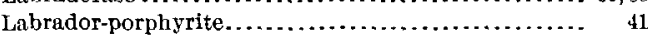

Labradorite-porphyry $\ldots \ldots \ldots \ldots \ldots \ldots \ldots \ldots \ldots \ldots \ldots, \quad 51$

Labradorose.

Lacroix, A., diagrams used by, description of ........ 12-13

Lake Superior region, rocks from .................. 33

Lamarose........................................ 48

Lamprophyre......................... 48,49,86,87 syenitic.................................... 48

La Plata Mountains, rocks from ................... 40

Lappare ............................. $46-47,84-85$

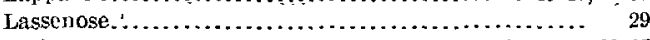

Latite.............................. 29,37,41,82,85

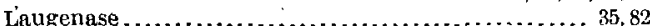

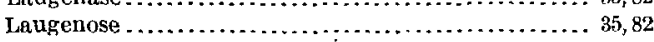

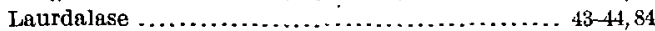

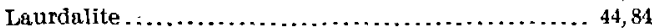

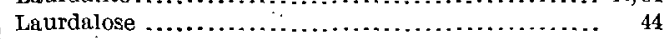

Laurvikite............................. $32,33,82$

Laurvikose . . . . . . . . . . . . . . . . . . . . . . . . . . . . $\$ 32-33$

Lebachose . ................. ................. 26

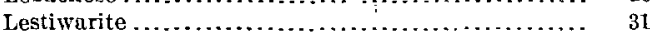

Leucite-absarokite.......................... 47,49

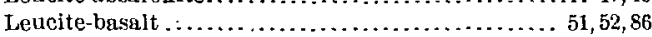

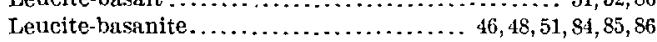

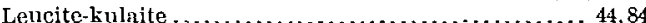

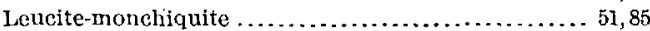

Léncite-nephelite lavas............................ 86,87

Leucite.nephelite-srenite $\ldots \ldots .44,84$ 


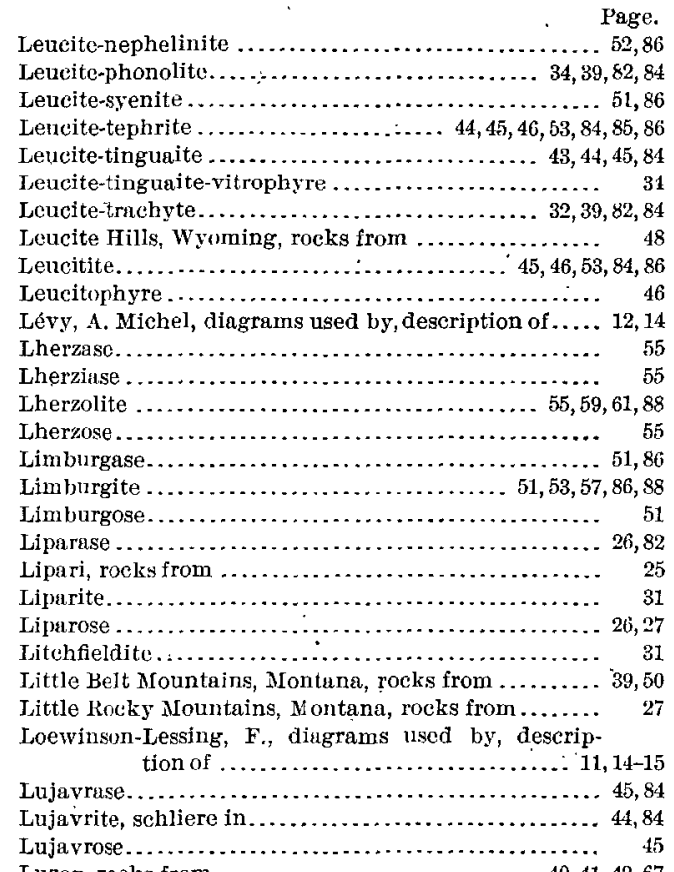
Luzon, rocks from $\ldots \ldots \ldots \ldots \ldots \ldots \ldots \ldots \ldots \ldots 40,41,42,67$

In.

Madagascar, rocks from.. 27,31, 33, 34, 40,42,44, 45, 51, 52, 67, 68 Madupite ................................. 58,86 Madupose.................................. 53

Magdeburgose

Magnetite-spinelite.

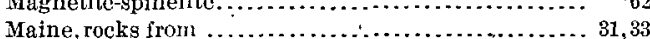

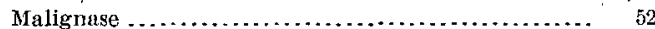

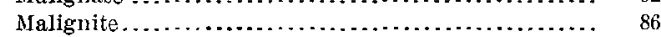

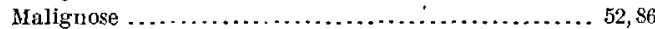

Maorare ................................. 59-61,88

Macriare.................................. 61,88

Mariciase ................................. 59

Maricose................................... 59

Mariposose...................................... 29

Mariupolite.............................. 35,82

Mariupolose ................................... 35

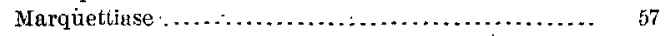

Marquettose.

Maryland, rocks from ........... 24, 26, 28, 30, 38, 43, 54, 60, 61 Marylundiare............................ 60,88 Mussuchusetts, rocks from... $26,27,28,31,34,39,44,45,49,54,67$ Moluphyre.................................. 47,87 Mexico, rocks from .......................... 45

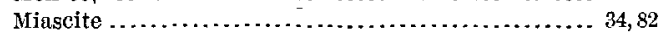
Miaskase................................. 34-35, 82 Miaśkose................................... 34 Mics-andesite.

Mica-basalt

Mica-ducite.

Mica-diorite

Nicu-gabbro

Micn-peridotite .......................... 57,88

Mica-picrophyre....................... 48,8

Mica-porphyry.

Mica-trachyte.
Page. Michel-Lévy, A., diagrams used by, description of ... 12,14 Michigan, rocks from............... 41,43,49,50,55,57 Microditroite ................................ 34 Microessexite............................... 45

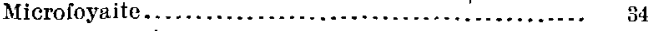

Micromonzonite .............................. Mihalose................................... 24 Minette.................................. 40,84 Minnesota, rocks from....... 27, 29, 30,33,36,41, 43, 49, 54, 62 Minnesotase ................................ 54 Minnesotiare .............................. 54,88 Minnesotiase . ................................ 54 Missouri, rocks from....................... 26, 27,67

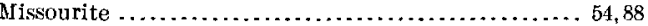

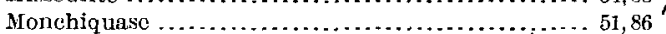
Monchiquite................. 44,50,51,52,84,86,87

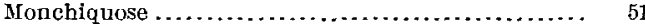

Montana, rocks from ........ 23, 24, 26, 27, 28, 29, 31, 33, 34, 36 $37,39,40,41,42,43,44,45,48,49,50,51,52,53,54,55,56,67$

Monthaldeite ............................... 41,85

Monzonase .......................................... 39,84

Monzonite...................... $37,40,41,42 ; 43,81,85$

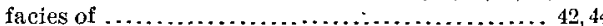

Monzonose ................................... $39-40$

Mount Ascutney, Vermont, rocks from.............. 67

Mïgge, 0 ., diagrams used by, description of ......... 15

Muscovite-granite ..............................

N.

Naples, rocks from

Nephelinite. .

$50,53,86$

Nephelite-aplite $\ldots \ldots \ldots \ldots \ldots \ldots \ldots \ldots \ldots \ldots \ldots \ldots .34,82$

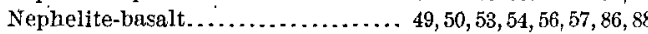

Nephelite-basanite ............................ 51, 86

Nephelite-felsite............................. 44,84

Nuphelite-gabbro............................... 51,86

Nephelite-malignite ..........................

Nephelite-melilite-basalt.................. 57,58,88

Nepheiite-monzonite ........................ 44,84

Nephelite-porphyry ....................... 45, 54,84,86

Nephelite-rhomben-porphyry ................. 35,82

Nepholite-sölvsbergite........................ $\quad 39$

Nephelite-syenite ............... 31, 32, 35, 39, 40,44, 82, 84

Nephelite-syonite-porphyry .................. 44

Nephelite-tephrite........................... 42, 51, 85

Nephelite-tinguaite . ......................... 35,82

Nerada, rocks from ........... 24, 25, 26, 28, 30,37,42,49,67

New Hampshire, rocks from................ $26,27,39,51$ New Jersey, rocks from.................. 34, 35, 46, 50

New Mexico, rocks from ............... 26,29, 31, 42,50,67

New South Wales, rocks from .................. 35

New York, rocks from..... 33, 41, 42, 43, 49, 55, 56, 58, 59, 62, 68

New Zealand, rocks from..................... 23, 24,61

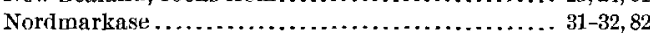

Nordmarkite $\ldots \ldots \ldots \ldots \ldots \ldots \ldots \ldots \ldots \ldots \ldots 28,31,32,39,82,84$

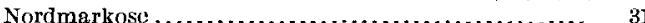

Norgare............................ 43-45, 84-85

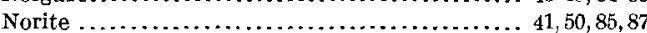

North America, average rock from............. 38

North Carolina, rocks from.................. 23,60,61

Norway, rocks from.................... 23, 26, 27, 28, 29 , $31,32,33,34,35,36,39,40,41,42,44,45,47,49,51,52,56,58$ Noyangose................................ 27

\section{O.}

Obsidian .......................... 28, 26, $28,29,82$

Odenwald, rocks from ....................... 24,50

Oligoclasite.............................. 29 
Page.

Olivine-diabase...................... 42, 47, 50,85,86 Olivine-diabase-porphyrite ................... 47 Olivine-essexite............................ 44 Olivine-gabbro. ... . ................ 43,50,54,56,85 Olivine-gabbro-diabase .................. 47,51,87 Olivine-laurdalite ......................... 44 Olivine-monzonite. ....................... 49,86 Omeose

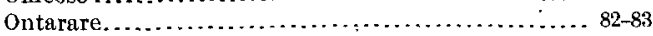
Ontario, rocks from.................. $33,52,58,59,62$ Oregon, rocks from . ..........................42,60 Orendase ............................. 48,86 Orendite . . .................................. 48,80

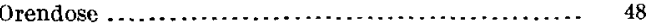

Ornose.

Osann, A., diagrams used by, description of ........... 15-16

Ourose

$\mathbf{P}$.

Paisanite

Paleolimburgite

Pantellaria, Italy, rocks from $\ldots \ldots \ldots \ldots . . .32,35,36,47,49,50,67$

Pantellerase ............................... 36,85

Pantellerite....................... $35,47,84,86$

Pantellerose ............................... $\quad 36$

Paolase ....................................... 56

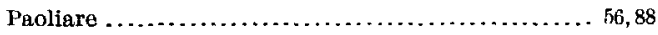

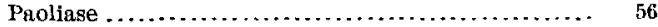

Paolose ...................................... 56

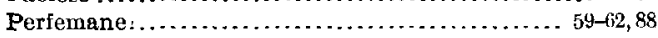

Pergamon rocks from

Peridotite $\ldots \ldots \ldots \ldots \ldots \ldots \ldots \ldots \ldots \ldots \ldots .54,55,57,60,86,88$

perlite .................................... 23

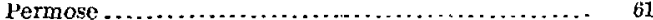

Persalane................................ $23-35,82-88$

Philippine Islands, rocks from ............40,41,42,67

Phlegrose ..........................................

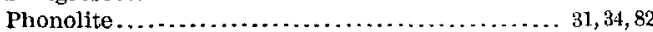

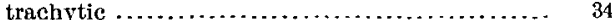

Piedmont, Italy, rocks from................... 42

Pikes Peak district, Colorndo, rocks from......... 25,51,67

Pirsson, L. V., diagrams used by, deseription of ........ 11

Pitchstone $\ldots \ldots \ldots \ldots \ldots \ldots \ldots \ldots \ldots \ldots \ldots \ldots \ldots 23,26,27,29$ rhyolitic ................................... 27

Placerose ................................. 38

Porphyrite....................... 29, $30,36,37,40$

Porphyrite-diorite ........................... 30

Porphyry ............................... 23, 27

Portugal, rocks from. . . . . . . . . . . . .

Portugare ......................................

Prussia, rocks from ............... 26, $31,36,40,48,49,51$

Pseudoleucite-syenite .......................... 48,84

Pulaskase . ............................... 32-38, 82

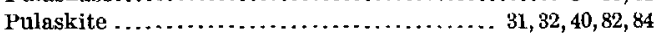

Pulaskose.

Puy de Dome, France, rocks from ............... 32

Py renees, rocks from . . . . . . . . . . . . . . . 43, 50, 55, 58

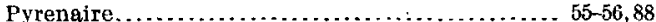

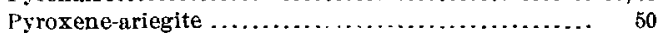

Pyroxene-porphyry.

Pyroxenite $\ldots \ldots \ldots \ldots \ldots \ldots \ldots \ldots \ldots \ldots \ldots \ldots \ldots \ldots \ldots, 56,57,59,60,88$

Q.

Quartz-banakite

Quartz-basalt. $38,41,49,85,86$

Quart2-diorite
Prge.

Quartz-diorite-nplite..........................$_{30}$ Quartz-diorite-porphyry $\ldots \ldots \ldots \ldots \ldots \ldots \ldots \ldots \ldots 25,30,83,85$

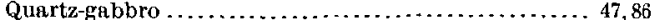
Quartz-keratophyre ...................... 24, 27,82 Quartz-lindoite $\ldots \ldots \ldots \ldots \ldots \ldots \ldots \ldots \ldots \ldots \ldots \ldots, \quad 29$ Quartz-mica-diorite..................... $30,36,37,84$ Quurtz-monzonite $\ldots \ldots \ldots \ldots \ldots \ldots \ldots \ldots \ldots 28,29,37,82,85$

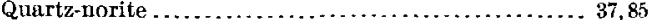
Quartz-orthoclasite ......................... $\quad 26$ Quartz-pantellerite....................... 27,82

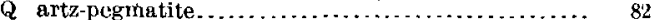
Quartz-porphyrite ....................... 29, 30 Quartz-porphyry $\ldots \ldots \ldots \ldots \ldots \ldots \ldots \ldots \ldots .23,24,26,27,28,82$ Quartz-pyroxene-diorite ....................... 37 Quartz-sölvsbergite .......................... $\quad 31$ Quartz-syenite $\ldots \ldots \ldots \ldots \ldots \ldots \ldots \ldots \ldots \ldots \ldots \ldots 27, \mathbf{3 1}, \mathbf{3 2}, 82$ Quartz-syenite-porphyry $\ldots \ldots \ldots \ldots \ldots \ldots \ldots \ldots .27,28,31,82$ Quebec, rocks from $\ldots \ldots \ldots \ldots \ldots \ldots \ldots \ldots \ldots 31,32,40,42,44$

\section{R.}

Rapakiwi granite.......................... 20. Reid, J. A., diagrams used by, description of ......... If Reyer, E., diagrams used by, dscription of . ......... 10 Rhode Island, rocks from ...................... 58,62 Rhombenporphyry. . . . . . . . . . . . . . . . $32,33,44,82$ Rhyolite....................... $23,24,26,27,28,29,82$. Rhyolite-perlite ............................ 28 Rhyolitic pitchstone. . . . . . . . . . . . . . . . . . . . . 27 Rhyolotrachyte ........................... 28 Ríesenase . . . . . . . . . . . . . . . . . . . . . . . . . . . 25, 88 Riesengebirge, Silesiu, rocks from.......... 24, 25, 28, 29 Riesenose................................ 25 Rockall Islund, rocks from . ................... Rockallase. . . . . . . . . . . . . . . . . . . . . . . . 47,86 Rockallite ............................ 47,86 Rockallose.................................. 47 Rosenbusch, H., diagrams used by, reference to...... 13 rock analyses eited from work by, reference to.... 21 Rossweinose ............................ 55 Russare.......................................... 34,83 Russia, rocks from ............... 27, b1, $38,35,42,84,60$

\section{S.}

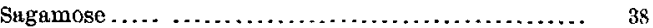
Salemase $\ldots \ldots \ldots \ldots \ldots \ldots \ldots \ldots \ldots \ldots \ldots \ldots \ldots \ldots \ldots .45,85$

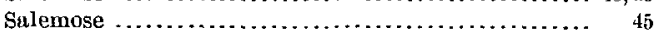
Salfemane .............................. $47-54$

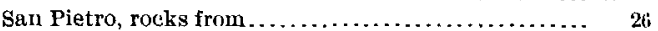
Sardinia, rocks from ........................ 26, 34

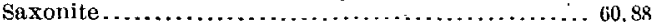
Saxony, rocks from...................... $31 ; 45$ Schwarzwald, rocks from . . . . . . . . . . . . . . . . 27 Schliere in granite......................... 25,37, 38

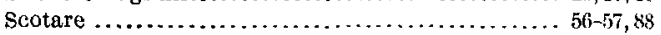
Scotland, rocks from.................... 28,38,44,49

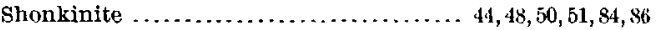
Shonkinite-monzonite....................... 40

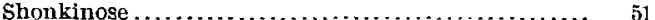
Shoshonite $\ldots \ldots \ldots \ldots \ldots \ldots \ldots \ldots \ldots \ldots \ldots \ldots .40,41,84,85$ Shoshonose $\ldots \ldots \ldots \ldots \ldots \ldots \ldots \ldots \ldots \ldots \ldots \ldots \ldots \ldots, .41$ Siberia, rocks from .......................... 26, 34 Sicily, rocks from $. \ldots \ldots \ldots \ldots \ldots \ldots \ldots \ldots \ldots \ldots \ldots . .85,43$ Silesia, rocks from .............. 24, 25, 28, 29, 36, $37,38,56$ Silver Cliff, Colorado, rocks from................ Similarity of rocks from diffevent regions, table showing.............................. $66-67$

$14474-$ No. $18-03--7$ 


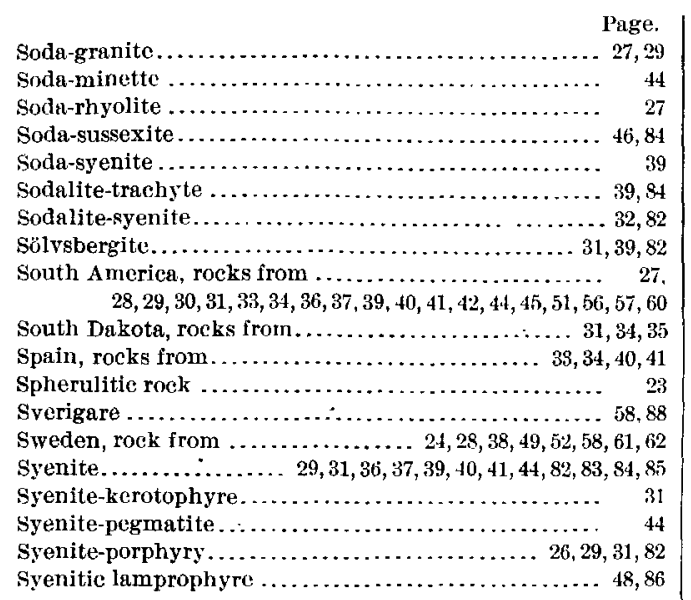

\section{T.}

Tachylyte .......................... 49 Tasmanare .............................. $3,82-88$

Teall, J. J.H., and Dakyns, J. R., diagrams used by... 10

Tehamos

Tephrite.

Tephritic trachyte.

Texas, rocks from $\ldots \ldots \ldots \ldots \ldots \ldots \ldots \ldots \ldots \ldots \ldots 26,27,57$

Texase.......................................

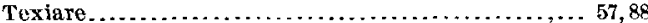

Theralite ......................... $44,52,53,84,86$

Thïringerwald, rock from

Thuringia, rocks from .........................

Tinguaite $\ldots \ldots \ldots \ldots \ldots \ldots \ldots \ldots \ldots 31,34,35,43,44,45,46,82,84$

Tonalase................................. 37-38, 85

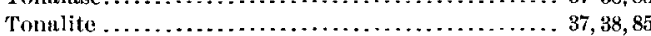

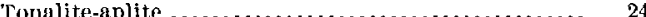

'Tonalose................................... 37-38

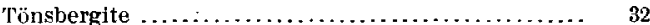

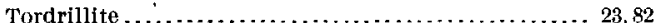

Toscanase . . . . . . . . . . . . . . . . . . . . . . . $27-29,82$

Toscanite .......................... 25, 27,29,82,83

Toscanose.............................. 28-29

Trachydolerite.$\ldots \ldots \ldots \ldots \ldots \ldots \ldots \ldots \ldots \ldots \ldots \ldots \ldots$, 82

Trachyte $\ldots \ldots \ldots \ldots, 28,29,30,31,32,37,89,40,43,82,83,84,85$ tephritic ............................. 33,40 Trachyte-andesite $\ldots \ldots \ldots \ldots \ldots \ldots \ldots \ldots \ldots \ldots \ldots \ldots, 36,84$

Trachytic phonolite

Transitional types, discussion of ................. 64-65

Transvaal, rocks from $\ldots \ldots \ldots \ldots \ldots \ldots \ldots \ldots \ldots \ldots .44,59$

Transylvania, rooks from..................... 34

Tiunlumnose ............................. 32

T! rol, rocks from.... 26, 27,37, 38, 40,41, 42, 43, 44, 45, 51, 53, 58

U.

Umptekase . . . . . . . . . . . . . . . . . . . . . 39,85 Umptekite.............................. 31, 39,82,84 Umptekuse .............................. 39

Unilaska, rocks from........................
Page.

Ural Mountains, rocks from $\ldots \ldots \ldots \ldots 27,31,54,56,60,61,68$

Urtase $\ldots \ldots \ldots \ldots \ldots \ldots \ldots \ldots \ldots \ldots \ldots \ldots \ldots \ldots .46-47,84$

Urtite.............................. $47,84,85$

Urtose ............................... 47

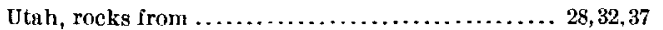

Uvaldiase................................... 57

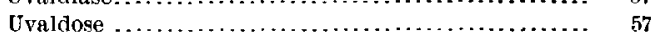

V.

Vaglare................................. 47,86 (6) 8

Vaalase .................................47,86

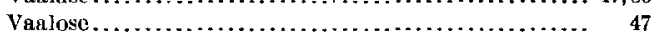

Variations in composition of rocks, discnssion of..... 63-64

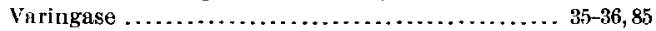

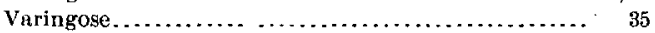

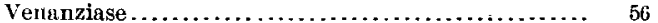

Venanzite ................................ 56,88

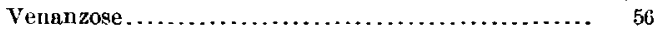

Verde, Cape, rocks from.................... 62, 63

Vermont, rocks from .......... 26, 27, 29, 31, 40,42, 49, 50,67, 68

Vesuvase $\ldots \ldots \ldots \ldots \ldots \ldots \ldots \ldots \ldots \ldots \ldots \ldots \ldots \ldots \ldots \ldots, 46,84$

Vesuvius, rocks from ...................... 45,46,53

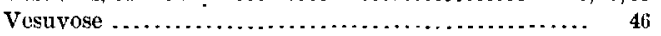

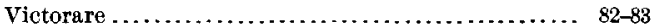

Victoria, Anstralia, rocks from.......... 23, 26, 27, 28, 35, 37

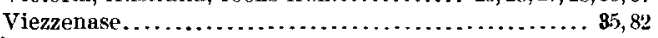

Viezzenose................................... 85

Vogesite ..................................... 86

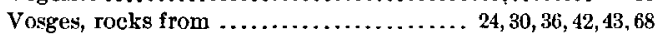

Vulcanite................................... 25,83

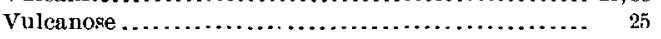

Vulsinite $\ldots \ldots \ldots \ldots \ldots \ldots \ldots \ldots \ldots \ldots \ldots \ldots \ldots \ldots, 32,41,82,85$

Vulsinose ..................................... 32

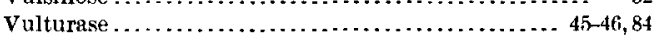

Vulturose ................................ 46

W.

Wales, rocks from $\ldots \ldots \ldots \ldots, 23,41$

Washington, D. C., rocks from................. 47

Washington, H. S., acknowledgments to.......... 22,65

cited on composition of the average rock ........ $\quad 66$

diagrams used $b y$, reference to ............... 11

rock analyses cited from paper by, reference to ... 21

Websterase $\ldots \ldots \ldots \ldots \ldots \ldots \ldots \ldots \ldots \ldots \ldots \ldots \ldots \ldots . .69 .6 \ldots$

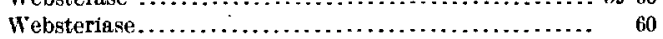

Websterite ................................. 60,88

Websterose $\ldots \ldots \ldots \ldots \ldots \ldots \ldots \ldots \ldots \ldots \ldots \ldots \ldots \ldots, 6_{60}$

Wehrlase ..................................... 55

Wehrliase.................................. 55

Wehrlite $\ldots \ldots \ldots \ldots \ldots \ldots \ldots \ldots \ldots \ldots \ldots \ldots \ldots \ldots \ldots, 54,55,88$

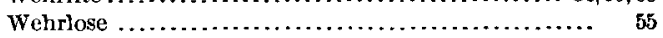

Westphalia, rock from................... $24,43,57$

Westphalose ............................ 24

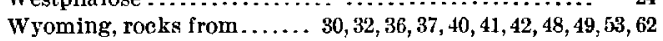

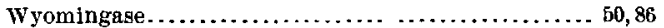

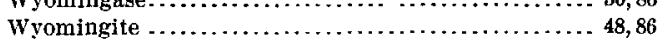

$\mathbf{Y}$.

Yellowstone Park, rocks from ................... 23 , $24,26,27,28,29,30,36,37,38,40,41,42,48,49,67$ Yellowstonose .................................... 


\section{PUBLICATIONS OF UNITED STATES GEOLOGICAL SURVFY.}

[Professional Puper No. 18.]

The serial publications of the United States Geological Survey consist of (1) Annual Report;, (2) Monographs, (3) Professional Papers, (4) Bulletins, (5) Mineral Resources, (6) Water-Supply anml Irrigation Papers, (7) Topographic Atlas of the United States-folios and separate sheets thereof, (8) Geologic Atlas of the United States-iolios. thereof. The classes numbered 2, 7, and 8 are sold at cost of publication; the others are distributed free. A circular giving complete lists may he had on application.

The Bulletins, Professional Papers, and Water-Supply Papers treat of a variety of subjects, aud the total number issued is large. They have therefore been classified into the following series: A, Economic geology; B, Descriptive geology; C, Systematic geology and paleontology;.D, Petrography and mineralogy; E, Chemistry and physics; F; Geography; G, Miscellaneous; H, Forestry; I, Irrigation; J, Water storage; K, Pumping water; L, Quality of water; M, General hydrographic investigations; $N$, Water power; .0, Underground waters; P, Hydrographic progress reports. This paper is the twenty-fourth in Series D and the thirty-eighth in Series E, the complete lists of which follow. ( $\mathrm{B}=$ Bulletin, $\mathrm{PP}=$ Professional Paper.)

\section{SERIES D, PETROGRAPHY AND MINERALOGY.}

B 1. On hypersthene-andesite and on triclinic pyroxene in angitic rocks, by whitman Cross, with a geological sketeh of Buffalo Peaks, Colorado, by S. F. Emmons. 1883. 42 pp., 2 pls.

$B$. . On secondary enlargements of mineral fragments in certain rocks, by R. D. Irving und C. K. Vun Hise. 1884,56 ph., 6 pls. (Out of stock.)

B 12. A erystallographic study of the thinolite of Lake Lahontun, by F. S. Dana. 1884.34 pl., 3 pIs.

B 17. On the development of crystallization in the igneous rocks of Washoe, Nevada, with notes on the geology of the district, by Amold Hagne and J. P. Iddings. 1885. 44 pp. (Out of stock.)

B 20. Contributions to the mineralogy of the Rocky Mountains, by Whitman Cross and W. F. Hillebrand. 1885 , 114 pl. 1 pl. (Ont of stock.)

B 2s. The gabbros and associated hornblende rocks occurring in the neighborhood of Baltimore, Muryland, by G. H. Williams. 1886 . 78 pp., 4 pls. (Out of stock.)

B 38. Peridotite of Elliott County, Kentucky, by J. S. Diller: 1887. 31 pp., 1 pl.

B 59. The gubbros and associated rocks in Delawire, by F. D. Chester. $1890.45 \mathrm{pp} ., 1 \mathrm{pl}$.

B 61. Contributions to the mineralogy of the Pacific coast, by W. H. Melville and Waldemar Lindgren. $1590.40 \mathrm{pp} ., 3 \mathrm{l}$.

B 62. The greenstone-schist areas of the Menominee and Marquette regions of Mrichigan; a contribution to the subject of dynamic metamorphism in eruptive rocks, by G. F. Williams; with introduction by R. D. Irving. $1890.241 \mathrm{pp}$., 16 pls. (Out of stock.)

B 66. On a group of volcanic rocks from the Tewan Mountains, New Mexico, and on the accurrence of primary quartz in certain basalts, by J. P. Iddings. $1890.34 \mathrm{pp}$.

B 74. The minerals of North Carolina, by F. A. Genth. 1891. 119 pp. (Out of stock.)

B 79. A late volcanic eruption in northern California and its peculiar lava, by J. S. Diller. 1891.33 pp., 17 pls. (Ont of stock.)

B 84. Some lava flows of the western slope of the Sierra Nevada, California, by F. L. Rumsome. $189 \% .74$ pp., 11 pls.

B 107. The trap dikes of the Lake Champlain region, by J. F. Kemp and V. F. Masters. 1893. 62 pp., 4 pls. (Out of steck.)

B 109. The eruptive and sedimentary rocks on Pigeon Point, Minnesota, and their contact phenornena, by W. S. Buyley. 1893. $121 \mathrm{pp} ., 16 \mathrm{pls}$.

B 126. A mineralogical lexicon of Franklin, Humpshire, and Hampden counties, Massachusetts, by B. K. Emerson. 1895. 180 pp., 1 pl.

B 136. Volcanic rocks of South Mountuin, Pennsyivaniu, by Florence Buscom. 1896. 124 pp., 28 pls.

B 150. The educational series of rock specimens collected and distributed by the United States Geological surver, by $J$.S. Diller. 1898.400 pp., 47 pls.

B 157. The gneisses, gabbro-schists, and associated rocks of southwestern Minnesota, by C. W. Hall. 1899. 160 pp., 27 pls. l'P 3. Geology and petrography of Crater Lake National Park, by J. S. Diller and H. B. Patton. 1902.167 pp., 19 pls.

B 209. The geology of Ascutney Mountain, Vermont, by R. A. Dily. 1903.122 pp., 7 pls.

PI 14. Chemical anulyses of igneous rocks published from 1884 to 1900 , with a eritical discussion of the character and use of analyses, by H. S. Washington. 1903. $495 \mathrm{pp}$.

PP 18. Chemical composition of igneous rocks expressed by menns of dingrams. with reference to rock classification on a quantitative chemico-mineralogicul basis, by J, P. Iddings. $1903.98 \mathrm{pp.,} 8 \mathrm{pls.}$ 
B 9. Report of work done in the Washington laboratory during the fiscal year 1883-84, by F. W, Clarke and T. Mr. Chatari. 1884. $40 \mathrm{pp}$. (Out of stock.)

B 14. Electrical und magnetic properties of the iron carburets, by Carl Barus and Vincent. Stroulıal. 1885. 2238 pl. (Out of stoek.)

B 27. Report of work dont in the Division of Chemistry and Physics, mainly during the year 1884-85. 1886.80 pp.

13 32. Lists und analyses of the minerul springs of the United States (a preliminarystudy), by Albert C. I'eule. 1886. 235 pu. (Out of stock:)

B 35. I'hysical properties of the imon carhurets, by Carl Barus and Vincent strouhal. $1886.62 \mathrm{pp}$.

B 36. Subsidenec of fine solid particles in liquids, by Carl Barus. 1886. $58 \mathrm{pp.} \mathrm{(Out} \mathrm{of} \mathrm{stock.)}$

B 42. Report of work done in the Division of Chemistry and Physies, mainly during the fiscal year $1885-86,1, \mathrm{~F}$. W. Clarke, 188\%. 152 ple, i pl. (Out of stock.)

B 47. Analyses of waters of the Yellowstone National Park, with an account of the methods of unalyses om Iloyerd, by Frank Austin Gooch and James Edward Whitfield. 1888. 84 pp. (Quitof stock.)

13 52. Subaerial decay of rocks and origin of the red color of certain formations, by Israel Cook Russell. $1889 ., 65$ pl., 5 pls. (Out of stock.)

B 54. On the thermoelectric measurement of high temperatures, by Carl Barus. 1889. 313 pp., 11 pls. (Out of stoek.)

B 55. Report of work done in the Division of Chemistry and Physics, mainly during the fiscal year 1886-87, by F: W. Clarke. 1889. $96 \mathrm{pp.}$ (Out of stock.)

B 60. Report of work done in the Division of Chemistry and Physics, mainly during the fiscal year 1887-88. $1890 . \quad 174$ pp. (Out of stock.)

B 64. Report of work done in the Division of Chemistry and Physies, mainly during the fiscal year 1888-89, by F. W. Clarke. 1890. $60 \mathrm{pp}$.

B 68. Farthquakes in California in 1889, by James Edward Keeler, 1890.25 pp.'

B 73. The viscosity of solids, by Carl Barus. 1891. xii, 139 pp., 6 pls.

B 78. Report of work done in the Division of Chemistry and Physies, mainly during the fiscal year 18s9-90, by F. W. Clarke. 1891. $131 \mathrm{pp}$.

B 90. Report of work done in the Division of Chemistry and Physics, muinly during the fiscal yeur 1890-91, hy F. W. Clarke. 1892. 77 pP.

B !2. The eompressibility of liquids, by Carl Barus. $189^{2} .96 \mathrm{pp} ., 29 \mathrm{pls.}$

B 94. The mechanism of solid viscosity, by Carl Barus. $1892.138 \mathrm{pp}$.

B 95. Earthquakes in California in 1890 and 1891, by Edward Singleton Holden, 1892. 31 1p.

B 96. The volume thermodynamies of liquids, by Cari Barus. 1892. $100 \mathrm{pp}$.

B 108. High temperature work in igneous fusion and ebullition, ehiefly in relation to pressire, by carl Burus. 1s:3. 57 pp., 9 pls.

B 112. Earthquakes in Californiu in 1892, by Charles 1). Perrine. 1893. 57 pp.

B 113. Report of work done in the Division of Chemistry und Physies during the fiscal yenrs $1891-92$ and $1892-93$, by F. W. Clarke. 1893. 115 pl.

3 114. Earthquakes in California in 1893 , by Charles D. Perrine. 1894. 23 pp.

B 125. The constitution of the silicates, by F. W. Clarke. 1895. $100 \mathrm{pp.} \mathrm{(Out} \mathrm{of} \mathrm{sturk.)}$

B 129. Enrthquakes in Califomia in 1894, by Charles D. Perrine. 1895. 25 yp.

B 147. Farthquakes in California in 1895, by Charles D. Perrine. 1896. $23 \mathrm{IP}$.

B 148. Analyses of rocks, with a chapter on analytical methods, laboratory of the Uniterl stutes Geological survey, 1 kso to 1896, by F. W. Clarke and W. F. Hillebrand. 1897 . $306 \mathrm{pp.} \mathrm{(Ont} \mathrm{of} \mathrm{stock.)}$

3 155. Earthquakes in California in 1896 and 1897, by Charles D. Perrine. $1898.47 \mathrm{pp}$.

B 161. Earthquakes in California in 1898, by Charles D. Perrine. 1899. 31 pp., 1 pl.

B 167. Contributions to chemistry and mineralogy from the laboratory of the United States Geological survey; Frunk W. Clarke, Chief Chemist. 1900 . $166 \mathrm{pp.}$

B 168. Anulyses of rocks, laboratory of the United Sthtes Geological survey, 1880 to 1899, talsulated by F. W. Clarke. 1900. 308 pp. (Out of stock.)

B 176. Some principles and methods of rock analysis, by W. F. Hillebrand. 1900.114 p.

B 186. On pyrite and marcasite, by H. N. Stokes. 1900. $50 \mathrm{pp}$.

B 207. The action of ammonium chlorife upon silieates, by F. W. Glurke and George Steiger. 1902. 57 pu. (Oit of stock.)

P' 14. Chemicul analyses of igneous rocks published from 1884 to 1900 , with a critical discussion of the character and use of analyses, by H. S. Washington. $1903.495 \mathrm{Ip}$.

PP 18. Chemical composition of igneous rocks expressed by means of diugrums, with referejece lo rock elassification on u quantitative chemico-mineralogical busis, by J. P. Iddings. 1903. 98 112., 8 pls.

Correspondence should be addressed to-

\section{- The Direuron,}

United STATes Geolohion SuRvey,

Skpтzмнeк, 1903.

WashingtoN, "D. C. 